\title{
Instanton corrections to the universal hypermultiplet and automorphic forms on $S U(2,1)$
}

\author{
Ling Bao, Axel Kleinschmidt, Bengt E. W. Nilsson, \\ Daniel Persson and Boris Pioline
}

The hypermultiplet moduli space in Type IIA string theory compactified on a rigid Calabi-Yau threefold $\mathcal{X}$, corresponding to the "universal hypermultiplet," is described at tree level by the symmetric space $S U(2,1) /(S U(2) \times U(1))$. To determine the quantum corrections to this metric, we posit that a discrete subgroup of the continuous tree level isometry group $S U(2,1)$, namely the Picard modular group $S U(2,1 ; \mathbb{Z}[i])$, must remain unbroken in the exact metric — including all perturbative and non-perturbative quantum corrections. This assumption is expected to be valid when $\mathcal{X}$ admits complex multiplication by $\mathbb{Z}[i]$. Based on this hypothesis, we construct an $S U(2,1 ; \mathbb{Z}[i])$-invariant, non-holomorphic Eisenstein series, and tentatively propose that this Eisenstein series provides the exact contact potential on the twistor space over the universal hypermultiplet moduli space. We analyze its non-Abelian Fourier expansion, and show that the Abelian and non-Abelian Fourier coefficients take the required form for instanton corrections due to Euclidean D2-branes wrapping special Lagrangian submanifolds, and to Euclidean NS5-branes wrapping the entire CalabiYau threefold, respectively. While this tentative proposal fails to reproduce the correct one-loop correction, the consistency of the Fourier expansion with physics expectations provides strong support for the usefulness of the Picard modular group in constraining the quantum moduli space.

\subsection{The hypermultiplet moduli space of $\mathcal{N}=2$ supergravity}


1.3. Rigid Calabi-Yau threefolds and the Picard modular group

1.4. Automorphic forms for the Picard modular group 197

1.5. Eisenstein series and the exact universal hypermultiplet geometry

1.6. Outline

2. On the Picard modular group $S U(2,1 ; \mathbb{Z}[i])$

2.1. The group $S U(2,1)$ and its Lie algebra $\mathfrak{s u}(2,1)$ 203

2.2. Complex hyperbolic space

2.3. Relation to the scalar coset manifold $S U(2,1) /(S U(2) \times U(1))$

2.4. Coset transformations and subgroups of $S U(2,1) \quad 209$

2.4.1 Heisenberg translations. 209

2.4.2 Rotations. 210

2.4 .3 Involution. 210

2.5. The Picard modular group 211

3. Eisenstein series for the Picard modular group 213

3.1. Lattice construction and quadratic constraint 213

3.2. Poincaré series on the complex upper half plane 215

$\begin{array}{lll}\text { 4. Fourier expansion of } \mathcal{E}_{s}(\phi, \chi, \tilde{\chi}, \psi) & 217\end{array}$

4.1. General structure of the non-Abelian

Fourier expansion

4.2. First constant term

4.3. Solution of constraint and Poisson resummation 
4.4. Second constant term

4.5. Abelian Fourier coefficients

4.6. Non-Abelian Fourier coefficients

4.7. Functional relation

5. Instanton corrections to the universal hypermultiplet

5.1. Twistor techniques for quaternionic-Kähler spaces

5.1.1 On the twistor space of the tree-level universal hypermultiplet

5.2. Quantum corrected hypermultiplet moduli spaces in type IIA

5.3. On the contact potential and the

Picard-Eisenstein series

5.3.1 D2-brane instantons

5.3.2 NS5-brane instantons

6. Conclusions

Acknowledgments

Appendix A. Dirichlet series and Gaussian integers

A.1.Euler products and Dirichlet series

A.2. Structure of Gaussian primes

Appendix B. Spherical vector and $p$-adic Eisenstein series

B.1. Formal construction

B.2. Real and $p$-Adic spherical vector

B.3. Product over primes 
C.1. Analysis of the norm constraint

C.2. Rewriting the Abelian measure

\section{Introduction and summary}

String theory compactified on a manifold $\mathcal{X}$ typically leads to a low-energy effective action with an often large number of massless scalar fields valued in a moduli space $\mathcal{M}$. In general, the Riemannian metric on $\mathcal{M}$ is deformed by perturbative and non-perturbative quantum corrections, making it very difficult to determine the exact form of the quantum effective action. In this paper we study the particular case of compactifications of type IIA string theory on a rigid Calabi-Yau threefold $\mathcal{X}$ (i.e., with Betti number $\left.h_{2,1}(\mathcal{X})=0\right)$. In this case, the hypermultiplet part of the moduli space $\mathcal{M}$ is known to be described, at tree level in the string perturbative expansion, by the symmetric space $\mathcal{M}_{\mathrm{UH}}=S U(2,1) /(S U(2) \times U(1))$. We analyze the quantum corrections to this classical geometry, and in particular conjecture the form of D2-brane and NS5-brane instanton contributions. Before entering into the details, we begin by discussing some of the ideas leading up to our proposal.

\subsection{Rigid moduli spaces for $\mathcal{N} \geq 4$ and Eisenstein series}

For compactifications preserving $\mathcal{N} \geq 4$ supersymmetry in $D=4$, the moduli space is always locally a symmetric space $\mathcal{M}=G / K$, with $G$ being a global symmetry and $K$, the maximal compact subgroup of $G$, being a local R-symmetry. In particular, $\mathcal{M}$ has restricted holonomy group $K$ and is rigid (see, e.g., [1] for a nice discussion). Quantum corrections are encoded in the global structure of $\mathcal{M}$, given by a double coset

$$
\mathcal{M}_{\text {exact }}=G(\mathbb{Z}) \backslash G / K
$$


where $G(\mathbb{Z})$ is typically an arithmetic subgroup of $G$, known as the $S$-, $T$ - or $U$-duality group, depending on the context [2-4]. For example, M-theory compactified on $T^{7}$ (or type IIA/B on $T^{6}$ ) gives rise to $\mathcal{N}=8$ supergravity in four dimensions, whose exact moduli space is conjectured to be $E_{7(7)}(\mathbb{Z}) \backslash E_{7(7)} /\left(S U(8) / \mathbb{Z}_{2}\right)[2]$. In such cases, the quantum effective action is expected to be invariant under $G(\mathbb{Z})$, which gives a powerful constraint on possible quantum corrections.

This idea was exploited with great success in the seminal work [5] in the context of type IIB supergravity in 10 dimensions, where the exact coefficient of the higher-derivative $\mathcal{R}^{4}$-type corrections were proposed to be given by a non-holomorphic Eisenstein series $\mathcal{E}_{3 / 2}^{S L(2, \mathbb{Z})}$ as a function of the "axio-dilaton" $C_{(0)}+\mathrm{ie}^{-\phi}$, valued on the fundamental domain $\mathcal{M}=$ $S L(2, \mathbb{Z}) \backslash S L(2, \mathbb{R}) / S O(2)$ of the Poincaré upper half plane. This proposal reproduced the known tree-level and one-loop corrections $[6,7]$, predicted the absence of higher loop corrections, later verified by an explicit two-loop computation [8], and suggested the exact form of $\mathrm{D}(-1)$-instanton contributions, later corroborated by explicit matrix model computations $[9,10]$. From the mathematical point of view, perturbative corrections and instanton contributions correspond, respectively, to the constant terms and Fourier coefficients of the automorphic form $\mathcal{E}_{3 / 2}^{S} \dot{L}(2, \mathbb{Z})$. This work was extended to toroidal compactifications of M-theory, where the $\mathcal{R}^{4}$-type corrections were argued to be given by Eisenstein series of the respective U-duality group [11-13], predicting the contributions of Euclidean $\mathrm{D} p$-brane instantons and, when $n \geq 6$, NS5-branes. Unfortunately, extracting the constant terms and Fourier coefficients of Eisenstein series is not an easy task, and it has been difficult to put the conjecture to the test. Part of our motivation is to develop the understanding of Eisenstein series beyond the relatively well understood case of $G(\mathbb{Z})=S L(n, \mathbb{Z})$

\subsection{The hypermultiplet moduli space of $\mathcal{N}=2$ supergravity}

Compactifications with fewer unbroken supersymmetries $(\mathcal{N} \leq 2$ in $D=$ 4) lead to moduli spaces which are generically not symmetric spaces. An interesting example is type IIA string theory compactified on a CalabiYau threefold $\mathcal{X}$, leading to $\mathcal{N}=2$ supergravity in four dimensions coupled to $h_{1,1}$ vector multiplets and $h_{2,1}+1$ hypermultiplets. The moduli space locally splits into a direct product $\mathcal{M}=\mathcal{M}_{\mathrm{V}} \times \mathcal{M}_{\mathrm{H}}$, where $\mathcal{M}_{\mathrm{V}}$ is a $2 h_{1,1}$-dimensional special Kähler manifold and $\mathcal{M}_{\mathrm{H}}$ a $4\left(h_{2,1}+1\right)$-dimensional quaternion-Kähler manifold, respectively. $\mathcal{M}_{\mathrm{V}}$ encodes the (complexified) 
Kähler structure of $\mathcal{X}$, while $\mathcal{M}_{\mathrm{H}}$ encodes deformations of the complex structure. $\mathcal{M}_{\mathrm{V}}$ is exact at tree-level in the perturbative string expansion, and well understood thanks to classical mirror symmetry (see e.g. [14] for an extensive introduction). In this paper we focus on the less understood hypermultiplet moduli space $\mathcal{M}_{\mathrm{H}}$. Note however that upon further compactification on a circle, $\mathcal{M}_{\mathrm{V}}$ is extended to a $4\left(h_{1,1}+1\right)$-dimensional quaternionKähler manifold by the $c$-map, and the vector and hypermultiplet moduli spaces become equally complicated, being exchanged under T-duality along the circle [15].

Contrary to $\mathcal{M}_{\mathrm{V}}$, the hypermultiplet moduli space $\mathcal{M}_{\mathrm{H}}$ receives perturbative and non-perturbative corrections in the string coupling [16-20]. The non-perturbative corrections are due to Euclidean D2-branes wrapping special Lagrangian submanifolds in $\mathcal{X}$, as well as due to Euclidean NS5-branes wrapping the entire Calabi-Yau threefold [16]. ${ }^{1}$ It has been an outstanding problem to understand how these effects modify the geometry of the moduli space $\mathcal{M}_{\mathrm{H}}$, mainly due to the fact that quaternion-Kähler geometry is much more complicated than special Kähler geometry. Recently, however, it has become apparent that twistor techniques can be efficiently applied to quaternion-Kähler geometry. In particular, deformations of the quaternionKähler geometry of $\mathcal{M}_{\mathrm{H}}$ are in one-to-one correspondance with deformations of its twistor space $\mathcal{Z}_{\mathcal{M}_{\mathrm{H}}}$, a $\mathbb{C} P^{1}$ bundle over $\mathcal{M}_{\mathrm{H}}$ [22-24] (see [25-29] for a physics realization of this equivalence). One virtue of this approach is that, contrary to $\mathcal{M}_{\mathrm{H}}$, the twistor space $\mathcal{Z}_{\mathcal{M}_{\mathrm{H}}}$ is Kähler, and therefore quantum corrections to $\mathcal{M}_{\mathrm{H}}$ can in principle be described in terms of the Kähler potential on its twistor space $\mathcal{Z}_{\mathcal{M}_{\mathrm{H}}}$. Furthermore, $\mathcal{Z}_{\mathcal{M}_{\mathrm{H}}}$ being a complex contact manifold, it can be described by holomorphic data, namely a set of complex symplectomorphisms between complex Darboux coordinate patches.

Using these techniques, much headway has been made in summing up part of the instanton corrections to hypermultiplet moduli spaces in both type IIA and IIB string theory [30-33]. These techniques were combined with the $S L(2, \mathbb{Z})$-invariance of the four-dimensional effective action in [30], to obtain the quantum corrections to $\mathcal{M}_{\mathrm{H}}^{\mathrm{IBB}}$ due to $\mathrm{D}(-1), \mathrm{F} 1$ and $\mathrm{D} 1$ instantons. $^{2}$ In this context, the Eisenstein series $\mathcal{E}_{3 / 2}^{S L(2, \mathbb{Z})}$, discussed above, reappears as the $\mathrm{D}(-1)$ instanton contribution to the Kähler potential on the twistor space of $\mathcal{M}_{\mathrm{H}}^{\mathrm{IIB}}$. This result was then mapped over to the IIA side using mirror symmetry [31], providing the quantum corrections to $\mathcal{M}_{\mathrm{H}}^{\mathrm{IIA}}$

\footnotetext{
${ }^{1}$ See [21] for a recent analysis of these effects in heterotic compactifications.

${ }^{2}$ The holomorphic action of $S L(2, \mathbb{Z})$ on the twistor space $\mathcal{Z}_{\mathcal{M}_{\mathrm{H}}}$ has been recently clarified in [33].
} 
from Euclidean D2-branes wrapping $A$-cycles in $\mathcal{X}$. Subsequently, the contribution from D2-branes wrapping arbitrary combinations of $A$ and $B$-cycles was obtained to linear order by covariantizing the result of [31] under "electric-magnetic duality" between $A$ - and $B$-cycles in the Calabi-Yau $[28,32]$. By the T-duality argument mentioned above, this also provides the contributions of $4 \mathrm{D}$ BPS black holes to the vector multiplet moduli space in type IIA or IIB string theory compactified on $\mathcal{X} \times S_{1}$. However, the NS5-brane contributions (or, on the vector multiplet side, the Kaluza-Klein monopole contributions) have so far proven to be considerably more elusive, although they can be in principle reached following the "roadmap" proposed in [31]. By postulating invariance under a larger discrete group $S L(3, \mathbb{Z})$, a subset of the NS5-brane contributions corresponding to the "extended universal hypermultiplet" was conjectured in [34]. This analysis (and presumably also the analysis in [30]) breaks down for rigid Calabi-Yau threefolds, the sector which we address in this work.

\subsection{Rigid Calabi-Yau threefolds and the Picard modular group}

In the present paper, we study the hypermultiplet moduli space $\mathcal{M}_{\mathrm{H}}$ in a restricted setting, namely for type IIA string theory compactified on a rigid Calabi-Yau threefold $\mathcal{X}$ (i.e., with $h_{2,1}(\mathcal{X})=0$ ). By the T-duality argument indicated above, our analysis applies equally well to the vector multiplet moduli space in type IIB string theory compactified on $\mathcal{X} \times S_{1}$. Rigid Calabi-Yau threefolds are rare, but examples can be found in the mathematics (see, e.g., [35]) and the physics literature (see, e.g., [36-39]). One of their peculiarities is that they do not admit a mirror in the usual sense, since $h_{1,1} \geq 1$ for any Calabi-Yau threefold. ${ }^{3}$ Thus, it is no longer clear that $\mathcal{M}_{\mathrm{H}}$ should admit an isometric action of $S L(2, \mathbb{Z})$. Moreover, rigid Calabi-Yau threefolds do not admit a K3 fibration, so are not amenable to heterotic/type II duality [40].

For such rigid Calabi-Yau threefolds then, the hypermultiplet sector consists solely of the "universal hypermultiplet," given at tree-level by the quaternion-Kähler symmetric space $\mathcal{M}_{\mathrm{UH}}(\mathcal{X})=S U(2,1) /(S U(2) \times U(1))$. The metric on $\mathcal{M}_{\mathrm{UH}}(\mathcal{X})$ is obtained via the $c$-map procedure from the complex structure moduli space of $\mathcal{X}[15,41]$. Since $\mathcal{X}$ has no complex structure

\footnotetext{
${ }^{3}$ It is possible that the superconformal field theory on $\mathcal{X}$ admits a mirror description as a Landau-Ginzburg model LG, but it is not obvious that this equivalence should extend at the non-perturbative level. Put differently, it is unclear whether type IIA on LG can still be lifted to M-theory, and whether type IIB on LG still exhibits $S L(2, \mathbb{Z})$ symmetry.
} 
deformations, its prepotential $F(X)$ is determined from the special geometry relations

$$
X=\int_{\mathcal{A}} \Omega, \quad \frac{\partial F}{\partial X}=\int_{\mathcal{B}} \Omega
$$

to be quadratic, namely

$$
F(X)=\tau X^{2} / 2, \quad \tau:=\frac{\int_{\mathcal{B}} \Omega}{\int_{\mathcal{A}} \Omega},
$$

where $\Omega \in H^{3,0}(\mathcal{X})$ is the holomorphic 3 -form, $(\mathcal{A}, \mathcal{B})$ is an integral symplectic basis of $H_{3}(\mathcal{X}, \mathbb{Z})$ and $\tau$ is a fixed complex number, the period matrix. The $c$-map then leads to the metric $^{4}$

$$
d s_{\mathcal{M}_{\mathrm{UH}}}^{2}(\tau)=d \phi^{2}+e^{2 \phi} \frac{|d \tilde{\chi}+\tau d \chi|^{2}}{\Im \tau}+e^{4 \phi}(d \psi+\chi d \tilde{\chi}-\tilde{\chi} d \chi)^{2}
$$

In type IIA string theory compactified on $\mathcal{X}, e^{\phi}$ is the four-dimensional string coupling, $\psi$ is the NS-NS axion, dual to the 2 -form $B_{(2)}$ in $D=4$, and $(\chi, \tilde{\chi})$ are the periods of the Ramond-Ramond 3 -form $C_{(3)}$ :

$$
\chi=\int_{\mathcal{A}} C_{(3)}, \quad \tilde{\chi}=\int_{\mathcal{B}} C_{(3)} .
$$

In the dual-type IIB string theory on $\mathcal{X} \times S_{1}, e^{\phi}$ is instead the inverse radius of the circle in $4 \mathrm{D}$ Planck units, while $\chi, \tilde{\chi}$ are the components of the 10 dimensional Ramond-Ramond 4 -form $C_{(4)}$ on $H^{3}(\mathcal{X}, \mathbb{R}) \times S^{1}$ and $\psi$ is the NUT potential (the dual of the Kaluza-Klein gauge field).

Classically, the family of metrics (1.4), parametrized by $\tau$, are all locally isometric to the symmetric space $S U(2,1) /(S U(2) \times U(1))=\mathbb{C H}^{2}$ (see Section 2 for details). A standard choice is to take $\tau=i$, leading to the familiar form of the left-invariant metric on $\mathbb{C H}^{2}$. However, at the quantum level the choice of $\tau$ is not inoccuous. Indeed, the Ramond-Ramond scalars $(\chi, \tilde{\chi})$ are

\footnotetext{
${ }^{4}$ Concretely, this metric may be obtained from the general $c$-map metric given in Equation (4.31) of [28] by setting $h_{2,1}=0, F(X)=\tau X^{2} / 2$ and implementing the change of variables: $r=e^{-2 \phi}, \zeta^{0}=-2 \sqrt{2} \chi, \tilde{\zeta}_{0}=2 \sqrt{2} \tilde{\chi}, \sigma=8 \psi$.
} 
known [42] to parametrize the intermediate Jacobian

$$
J(\mathcal{X})=\frac{H^{3}(\mathcal{X}, \mathbb{C})}{H^{3}(\mathcal{X}, \mathbb{Z})}
$$

Equivalently, they are subject to discrete identifications

$$
(\chi, \tilde{\chi}) \rightarrow(\chi+a, \tilde{\chi}+b), a, b \in \mathbb{Z} .
$$

If $\mathcal{X}$ is a rigid Calabi-Yau threefold, its intermediate Jacobian is an elliptic curve

$$
J(\mathcal{X})=\mathbb{C} /(\mathbb{Z}+\tau \mathbb{Z})
$$

where $\tau$ is the period matrix defined in (1.3). Thus, different choices of $\tau$ lead to locally isometric but globally inequivalent metrics. In the present work we shall restrict to the particular choice ${ }^{5} \tau=i$, corresponding to rigid Calabi-Yau threefolds for which the intermediate Jacobian is a square torus $\mathbb{C} / \mathbb{Z}[i]$, where $\mathbb{Z}[i]$ denotes the Gaussian integers $\{z \in \mathbb{C} \mid \Re(z), \Im(z) \in \mathbb{Z}\}$, corresponding to the ring of integers in the imaginary quadratic number field $\mathbb{Q}(i)$. Mathematically, this choice implies in particular that $J(\mathcal{X})$ admits "complex multiplication," a notion that originates from the study of elliptic curves $\mathbb{C} /(\mathbb{Z}+\tau \mathbb{Z})$, which are said to admit complex multiplication (or are of "CM-type") if and only if the modular parameter $\tau$ takes values in an imaginary quadratic extension of $\mathbb{Q}$. Many but not all rigid Calabi-Yau threefolds admit complex multiplication; a necessary and sufficient criterion is that the intermediate Jacobian of the Calabi-Yau threefold is of CM-type (see, e.g., [35] for a review). An example of a rigid Calabi-Yau threefold that does not admit complex multiplication is provided by the hypersurface constructed in [43]. ${ }^{6}$ For examples of rigid Calabi-Yau threefolds that admit complex multiplication by $\mathbb{Z}[i]$, as is relevant in the present work, see [35].

While quantum corrections are bound to break the continuous isometric action of $S U(2,1)$ on the hypermultiplet moduli space $\mathcal{M}_{\mathrm{UH}}$, we posit that they preserve a discrete arithmetic subgroup $G(\mathbb{Z}) \subset S U(2,1)$ (note however

\footnotetext{
${ }^{5}$ We are grateful to Jan Stienstra for pointing out this assumption, which was implicit in the first version of this work. We comment on the possible duality symmetries for rigid Calabi-Yau compactifications with $\tau \neq i$ in Section 6 .

${ }^{6}$ We also note that complex multiplication has appeared previously in the physics literature in $[44,45]$.
} 
that we do not assume that $\mathcal{M}_{\mathrm{UH}}^{\text {exact }}$ is a double coset $\left.G(\mathbb{Z}) \backslash G / K\right) .{ }^{7}$ On physical grounds, the subgroup $G(\mathbb{Z})$ should contain the following action on the moduli:

1. A discrete Heisenberg group $N(\mathbb{Z})$, acting by discrete (Peccei-Quinn) shift symmetries on the axions $\chi, \tilde{\chi}$ and $\psi[47]$ :

$$
\begin{aligned}
& \chi \longmapsto \chi+a, \\
& \tilde{\chi} \longmapsto \tilde{\chi}+b, \\
& \psi \longmapsto \psi+\frac{1}{2} c-a \tilde{\chi}+b \chi,
\end{aligned}
$$

where $a, b, c \in \mathbb{Z}$, while leaving the dilaton $\phi$ invariant. In the type IIA setting, the breaking of the continuous shifts of $\chi$ and $\tilde{\chi}$ are due to D2-brane instantons, while the breaking of the shift of $\psi$ is due to the combined effects of D2- and NS5-brane instantons. The factor $1 / 2$ appearing in front of $c$ is in agreement with the quantization condition on the NS5-brane instantons derived in [47]. The Heisenberg symmetry (1.9) should hold more generally for any value of the period matrix $\tau$.

2. The "electric-magnetic duality" $R$ which interchanges the $\mathrm{R}-\mathrm{R}$ scalars $\chi$ and $\tilde{\chi}[47]$ :

$$
R:(\chi, \tilde{\chi}) \longmapsto(-\tilde{\chi}, \chi) .
$$

This symmetry is only expected to hold for rigid Calabi-Yau compactifications with $\tau=i$. In that case it amounts to a change of symplectic basis for $H_{3}(\mathcal{X}, \mathbb{Z})$.

3 . We further assume that a discrete subgroup $S L(2, \mathbb{Z})$ of the fourdimensional $S$-duality (or, on the type IIB side, Ehlers symmetry), acting in the standard non-linear way on the complex parameter $\chi+i e^{-\phi}$ on the slice $\tilde{\chi}=\psi=0$, is left unbroken by quantum corrections. As in earlier endeavors [48-52], it is difficult to justify this assumption rigorously, but the fact, demonstrated herein, that it leads to physically sensible results can be taken as support for this assumption. ${ }^{8}$

${ }^{7}$ The fate of the continuous symmetry $S U(2,1)$ at the level of higher derivative corrections to the low energy effective action will be analyzed in a follow-up paper [46].

${ }^{8}$ On non-rigid Calabi-Yau manifolds one can use mirror symmetry to obtain S-duality transformations in IIA from the $S L(2, \mathbb{Z})$ symmetry of type IIB, as has been advocated in literature for example in [19, 53-55]. 
Based on these assumptions, it follows that, when $\tau=i, G(\mathbb{Z})$ must be the Picard modular group ${ }^{9} S U(2,1 ; \mathbb{Z}[i]$ ), defined as the intersection (see, e.g., $[56,57])$ :

$$
S U(2,1 ; \mathbb{Z}[i]):=S U(2,1) \cap S L(3, \mathbb{Z}[i])
$$

Indeed, the symmetries $1,2,3$ above reproduce the list of generators of the Picard modular group obtained in [56]. We find it remarkable that adjoining electric-magnetic duality and S-duality to the physically well-established Peccei-Quinn shift symmetries generates an interesting discrete subgroup of $S U(2,1)$.

We conclude this section by emphasizing that although the moduli $\{\phi, \chi, \tilde{\chi}, \psi\} \in \mathcal{M}_{\mathrm{UH}}$ occur universally for any Calabi-Yau threefold, the "universal hypermultiplet moduli space" (1.4) is not a universal subsector of the hypermultiplet moduli space $\mathcal{M}_{\mathrm{H}}(\mathcal{X})$ for non-rigid $\mathcal{X}[1]$. (This is is in contrast to the "extended universal hypermultiplet sector" introduced in [34].) However, in cases where $\mathcal{M}$ is a symmetric space, it can often be written as a fiber bundle over $\mathcal{M}_{\mathrm{UH}}$. One example is type II string theory compactified on $T^{7}$, where the moduli space can be written as the fiber bundle $[1,15]$

$$
\frac{E_{7(7)}}{S U(8) / \mathbb{Z}_{2}} \rightarrow \frac{S U(2,1)}{S U(2) \times U(1)} \times \frac{S L(2, \mathbb{R})}{S O(2)}
$$

In this context, it would be interesting to investigate which discrete subgroup of $S U(2,1)$ is singled out by the intersection with the U-duality group $E_{7(7)}(\mathbb{Z})[2]$. A similar decomposition as (1.12) also occurs for very special $\mathcal{N}=2$ supergravity theories, where the second factor on the r.h.s. is replaced by a non-compact version of the five-dimensional U-duality group [58,59]. Thus, it is plausible that the considerations in this paper may have bearing on the more general case of non-rigid Calabi-Yau threefolds.

\subsection{Automorphic forms for the Picard modular group}

Having identified (1.11) as a candidate symmetry group, we apply standard machinery to construct a simple automorphic form $\mathcal{E}_{s}(\mathcal{K})$ of $S U(2,1 ; \mathbb{Z}[i])$,

\footnotetext{
${ }^{9}$ The nomenclature "Picard group" is not unique, in fact our Picard group is a member of a family of similar groups $P S U(1, n+1 ; \mathbb{Z}[i])$ of which the case $n=0$, corresponding to $P S L(2, \mathbb{Z}[i])$ is also often called the Picard group. In this paper we will always mean $S U(2,1 ; \mathbb{Z}[i])$ when speaking of the Picard group.
} 
which satisfies

$$
\Delta_{\mathbb{C H}^{2}} \mathcal{E}_{s}(\phi, \lambda, \gamma)=s(s-2) \mathcal{E}_{s}(\phi, \lambda, \gamma),
$$

where $\Delta_{\mathbb{C H}^{2}}$ is the Laplace-Beltrami operator on $\mathbb{C H}^{2}$, given in (3.9) below. For the sake of completeness, and because they mutually enlighten each other, we shall present three equivalent constructions of the Eisenstein series for the Picard modular group:

- First, we generalize the construction of non-holomorphic Eisenstein series for real classical groups over the integers in [13] to unitary groups over the Gaussian integers, and consider the constrained lattice sum

$$
\mathcal{E}_{s}(\mathcal{K}):=\sum_{\substack{\vec{\omega} \in \mathbb{Z}[i]]^{3} \\ \vec{\omega}^{\dagger} \cdot \eta \cdot \vec{\omega}=0}}^{\prime}\left[\vec{\omega}^{\dagger} \cdot \mathcal{K} \cdot \vec{\omega}\right]^{-s}
$$

where $\mathcal{K}=\mathcal{V} \mathcal{V}^{\dagger}$ is a Hermitian matrix parametrizing the coset space $S U(2,1) /(S U(2) \times U(1))$, and the sum runs over non-zero triplets of Gaussian integers $\vec{\omega}$ subject to a certain quadratic constraint (3.3). The prime indicates that $\vec{\omega}=(0,0,0)$ is excluded from the sum. More generally, throughout this paper a prime on a summation symbol will indicate that the zero value is excluded from the sum.

- Secondly, we consider the $S U(2,1 ; \mathbb{Z}[i])$-invariant Poincaré series

$$
\mathcal{P}_{s}(\mathcal{Z}):=\sum_{\gamma \in N(\mathbb{Z}) \backslash S U(2,1 ; \mathbb{Z}[i])} \mathcal{F}(\gamma \cdot \mathcal{Z})^{s},
$$

where $\mathcal{F}(\mathcal{Z})$ is a function on $S U(2,1) /(S U(2) \times U(1))$, which is manifestly invariant under the Heisenberg subgroup $N(\mathbb{Z}) \subset S U(2,1 ; \mathbb{Z}[i])$. As shown in Section 3, this construction yields the same automorphic form as before up to an $s$-dependent factor,

$$
\mathcal{E}_{s}(\mathcal{K})=4 \zeta_{\mathbb{Q}(i)}(s) \mathcal{P}_{s}(\mathcal{Z}),
$$

where $\zeta_{\mathbb{Q}(i)}(s)$ is the Dedekind zeta function for the Gauss field $\mathbb{Q}(i)$, defined in (4.15).

- Thirdly, in Appendix B we show that the same automorphic form can be obtained by the general adelic method explained, e.g., in [60-62],

$$
\mathcal{E}_{s}(\mathcal{V})=\sum_{\substack{\left(C_{1}, C_{2}\right) \in P \mathbb{Q}(i)^{2} \\\left|C_{1}\right|^{2}-2 \Im\left(C_{2}\right)=0}}^{\prime} \rho(\mathcal{V}) \cdot \prod_{g \text { prime }} f_{g}\left(C_{1}, C_{2}\right)
$$


where the infinite product runs over all Gaussian prime numbers including $g=\infty$. Here, $\mathcal{V}$ is a coset representative of $S U(2,1) /(S U(2)$ $\times U(1)), P \mathbb{Q}(i)^{2}$ is the projective space $\mathbb{Q}(i)^{3} / \mathbb{Q}(i)^{*}, \rho$ is the principal continuous series representation of $S U(2,1)$ on functions on $P \mathbb{Q}(i)^{2}$, $f_{\infty}:=f_{K}$ is the $S U(2) \times U(1)$-invariant spherical vector from [63], and $f_{g}(g<\infty)$ its $p$-adic counterpart.

Generally, any function $f$ on $\mathbb{C H}^{2}$ which obeys the following three conditions:

1. $f$ satisfies the Laplace equation $\Delta_{\mathbb{C H}^{2}} f=s(s-2) f$,

2. $f$ is invariant under the Heisenberg group $N(\mathbb{Z})$ (see $(1.9)$ ),

3. $f$ has at most polynomial growth in $e^{\phi}$ as $e^{\phi} \rightarrow 0$,

admits a non-Abelian Fourier expansion of the form ${ }^{10}$

$$
\begin{aligned}
f(\phi, \chi, \tilde{\chi}, \psi)= & A(s) e^{-2 s \phi}+B(s) e^{-2(2-s) \phi} \\
& +e^{-2 \phi} \sum_{\left(\ell_{1}, \ell_{2}\right) \in \mathbb{Z}^{2}}^{\prime} C_{\ell_{1}, \ell_{2}}^{(\mathrm{A})}(s) K_{2 s-2}\left(2 \pi e^{-\phi} \sqrt{\ell_{1}^{2}+\ell_{2}^{2}}\right) e^{-2 \pi i\left(\ell_{1} \chi+\ell_{2} \tilde{\chi}\right)} \\
& +e^{-\phi} \sum_{k \in \mathbb{Z}}^{\prime} \sum_{\ell=0}^{4|k|-1} \sum_{n \in \mathbb{Z}+\frac{\ell}{4|k|}} \sum_{r=0}^{\infty} C_{r, k, \ell}^{(\mathrm{NA})}(s)|k|^{1 / 2-s} e^{-4 \pi|k|(\tilde{\chi}-n)^{2}} \\
& \times H_{r}(\sqrt{8 \pi|k|}(\tilde{\chi}-n)) \\
& \times W_{-r-\frac{1}{2}, s-1}\left(4 \pi|k| e^{-2 \phi}\right) e^{8 \pi i k n \chi-4 \pi i k(\psi+\chi \tilde{\chi})},
\end{aligned}
$$

where $K_{s}, H_{r}$ and $W_{-r-\frac{1}{2}, s-1}$ denote the modified Bessel function, Hermite polynomial and Whittaker function, respectively. The first line in (1.18) corresponds to the "constant terms", i.e., the leading terms in an expansion at the cusp $e^{\phi} \rightarrow 0$. The second line is the Abelian contribution, corresponding to an ordinary Fourier expansion with respect to the Abelianized Heisenberg group $N / Z$, where $N$ is the Heisenberg subgroup of upper triangular matrices in $S U(2,1)$ and $Z$ is the center of $N$ (in this particular case it coincides with the commutator subgroup $[N, N])$. Finally, the last two lines represent the non-Abelian contribution, i.e., the part of $f$ which transforms non-trivially under the action of the center $Z$ of the Heisenberg group. We have chosen to write them in the " $\tilde{\chi}$-polarization", where the $a$ and $c$ shifts

\footnotetext{
${ }^{10} \mathrm{~A}$ similar expansion was given in [64] but we disagree with the details in this reference. For instance, the second constant term appears to be missing in Theorem 5.3.1ii) of [64]. Such a term is required on general grounds [65].
} 
in (1.9) are diagonalized, but it is also possible to use the " $\chi$-polarization," where $b$ and $c$ shifts are diagonalized (see Section 4.1 for a detailed discussion of the choice of polarization). The constant terms $A(s), B(s)$ and the numerical Fourier coefficients $C_{\ell_{1}, \ell_{2}}^{(\mathrm{A})}(s)$ will be derived in Section 4 .

Our main mathematical results may now be summarized in the following propositions and conjecture:

Proposition 1.1 (Constant terms). The constant terms in the Fourier expansion of the Eisenstein series $\mathcal{E}_{s}(\phi, \chi, \tilde{\chi}, \psi)$ in Equation (1.18) are given by

$$
\mathcal{E}_{s}^{\text {(const) }}=4 \zeta_{\mathbb{Q}(i)}(s)\left\{\mathrm{e}^{-2 s \phi}+\frac{\mathfrak{Z}(2-s)}{\mathfrak{Z}(s)} \mathrm{e}^{-2(2-s) \phi}\right\} .
$$

Here we have defined the "Picard zeta function" $\mathfrak{Z}(s)$ through

$$
\mathfrak{Z}(s):=\zeta_{\mathbb{Q}(i) *}(s) \beta_{*}(2 s-1)
$$

where $\zeta_{\mathbb{Q}(i) *}(s)$ and $\beta_{*}(2 s-1)$ are the completed Dedekind zeta function (4.16) and Dirichlet beta function (4.22), respectively.

This proposition is proven in Sections 4.2 and 4.4 .

Proposition 1.2 (Abelian Fourier coefficients). The numerical Fourier coefficients $C_{\ell_{1}, \ell_{2}}^{(A)}(s)$ in the expansion of $\mathcal{E}_{s}(\phi, \chi, \tilde{\chi}, \psi)$ with respect to the abelianized Heisenberg group $N /[N, N]$ are given by

$$
C_{\ell_{1}, \ell_{2}}^{(A)}(s)=\frac{2 \zeta_{\mathbb{Q}(i)}(s)}{\mathfrak{Z}(s)}\left[\ell_{1}^{2}+\ell_{2}^{2}\right]^{s-1} \sum_{\omega^{\prime} \mid \Lambda}\left|\omega^{\prime}\right|^{2-2 s} \sum_{z \mid \frac{\Lambda}{\omega^{\prime}}}|z|^{4-4 s},
$$

where $\sum_{\omega^{\prime} \mid \Lambda}$ denotes a sum over Gaussian divisors of $\Lambda=\ell_{2}-i \ell_{1} \in \mathbb{Z}[i]$, and $\omega^{\prime}$ is a Gaussian integer such that $\operatorname{gcd}\left(\Re\left(\omega^{\prime}\right), \Im\left(\omega^{\prime}\right)\right)=1$.

This proposition is proven in Section 4.5 and Appendix C.

To have a complete understanding of the Fourier expansion one must also extract the non-Abelian numerical coefficients $C_{r, k, \ell}^{(\mathrm{NA})}(s)$, corresponding to the Fourier expansion of $\mathcal{E}_{s}$ with respect to the center $Z=[N, N]$ of the Heisenberg group $N$. Some preliminary results in this direction are presented in Section 4.6. 
Conjecture (Functional relation). The Eisenstein series $\mathcal{P}_{s}=$ $\left(4 \zeta_{\mathbb{Q}(i)}(s)\right)^{-1} \mathcal{E}_{s}$ satisfies the following functional relation:

$$
\mathfrak{Z}(s) \mathcal{P}_{s}=\mathfrak{Z}(2-s) \mathcal{P}_{2-s} .
$$

The map $s \mapsto 2-s$ corresponds to the action of the restricted Weyl group of $\mathfrak{s u}(2,1)$. The validity of $(1.22)$ at the level of the constant and Abelian terms holds true by the above propositions, but our incomplete understanding of the non-Abelian terms prevents us from stating it as a theorem. We note that a different functional relation for $\mathcal{P}_{s}$ was stated in [66] but it appears to be inconsistent with the constant terms (1.19).

\subsection{Eisenstein series and the exact universal hypermultiplet geometry}

As a test of the idea that the Picard modular group should control the quantum corrections to the universal hypermultiplet moduli space, we tentatively propose that the $S U(2,1 ; \mathbb{Z}[i])$-invariant Eisenstein series $\mathcal{E}_{s}(\phi, \chi, \tilde{\chi}, \psi)$, for the specific value $s=3 / 2$, gives a non-perturbative completion of the contact potential $e^{\Phi\left(x^{\mu}, z\right)}$ restricted to a certain holomorphic section $z=z\left(x^{\mu}\right)$ of the twistor space $\mathcal{Z}_{\mathcal{M}_{\text {UH }}}$ of the universal hypermultiplet moduli space. In this context, the constant terms of the Fourier expansion (1.18) represent the classical and one-loop contributions to the moduli space metric, respectively. Matching the power of the dilaton $e^{\phi}$ is what fixes the value of $s$ above. This proposal is tentative however, as it turns out that the numerical coefficient of the one-loop term predicted by (1.18) is inconsistent with its known value in string theory [17] (in particular, it has the wrong sign). We proceed nevertheless, since the structure of the non-Abelian Fourier expansion (1.18) is largely independent of the automorphic form under consideration.

In this scenario, the Abelian terms in (1.18) have the suitable form to describe the effects of D2-brane instantons wrapping supersymmetric 3 -cycles in the homology class $\ell_{1} \mathcal{A}+\ell_{2} \mathcal{B} \in H_{3}(\mathcal{X}, \mathbb{Z})$, where $\mathcal{A}$ and $\mathcal{B}$ provide a symplectic basis for $H_{3}(\mathcal{X}, \mathbb{Z})$. The Abelian Fourier coefficients $C_{\ell_{1}, \ell_{2}}^{(\mathrm{A})}(3 / 2)$, related to the instanton measure $\mu_{3 / 2}\left(\ell_{1}, \ell_{2}\right)$ via (4.69), should count supersymmetric cycles in the above holomogy class. The instanton measure $\mu_{3 / 2}$ $\left(\ell_{1}, \ell_{2}\right)$ generalizes the familiar $\mathrm{D}(-1)$ instanton measure $\mu_{3 / 2}(N)$ of $[5]$, which is also known to capture the effects of pure charge $N A$-type D2-brane instantons [31] (corresponding to $\ell_{2}=0$ ). On the other hand, non-Abelian terms have the suitable form to represent the effects of charge $k$ NS5-brane instantons, possibly bound to D2-branes. We take these encouraging facts 
as evidence that the Picard modular group should be a powerful principle in constraining the exact metric on the hypermultiplet moduli space in rigid Calabi-Yau compactifications, and in particular in determining the NS5-brane instantons which have remained elusive for generic Calabi-Yau compactifications. We comment on alternative choices of automorphic form for $S U(2,1 ; \mathbb{Z}[i])$, which may alleviate the shortcomings of the Eisenstein series $\mathcal{E}_{3 / 2}$ in Section 6 .

\subsection{Outline}

In Section 2 we give a detailed description of the group $S U(2,1)$, the symmetric space $S U(2,1) /(S U(2) \times U(1))$ and the Picard modular group $S U(2,1 ; \mathbb{Z}[i])$. In Section 3 we construct an $S U(2,1 ; \mathbb{Z}[i])$-invariant Eisenstein series $\mathcal{E}_{s}(\phi, \chi, \tilde{\chi}, \psi)$ in the principal continuous series of $S U(2,1)$ by two different methods. We proceed in Section 4 to compute the Fourier expansion of $\mathcal{E}_{s}$, extracting explicit forms for the constant terms as well as the Abelian and non-Abelian Fourier coefficients. Finally, in Section 5, we use the automorphic form $\mathcal{E}_{s}(\phi, \chi, \tilde{\chi}, \psi)$ at order $s=3 / 2$ to conjecture the exact form of the D2-brane and NS5-brane instanton corrections to the universal hypermultiplet moduli space $\mathcal{M}_{\mathrm{UH}}$. In Appendix A, we review some basic facts about Gaussian integers and Gaussian primes as well as a reminder of Dirichlet series. The third construction of the Eisenstein series $\mathcal{E}_{s}(\phi, \chi, \tilde{\chi}, \psi)$ using an adelic construction is given for completeness in Appendix B. This construction can be viewed as an extension of the analysis in Section 2 of [63] to the automorphic setting. In Appendix $\mathrm{C}$ we provide details on the derivation of the Abelian Fourier coefficients $C_{\ell_{1}, \ell_{2}}^{(\mathrm{A})}(s)$.

\section{On the Picard modular group $S U(2,1 ; \mathbb{Z}[i])$}

As indicated in the Introduction, a key character in this paper is the symmetric space $S U(2,1) /(S U(2) \times U(1))$. This space describes the tree-level moduli space of the universal hypermultiplet in type IIA string theory compactified on a rigid Calabi-Yau threefold. ${ }^{11}$ In this section, we set up notations for the group $S U(2,1)$, give two equivalent descriptions of the symmetric space $S U(2,1) /(S U(2) \times U(1))$ and introduce the Picard modular group $S U(2,1 ; \mathbb{Z}[i])$.

\footnotetext{
${ }^{11}$ We note that the coset space $S U(2,1) /(S U(2) \times U(1))$ also appears as the moduli space of the Einstein-Maxwell system when dimensionally reduced from $D=4$ to $D=3$ on a spacelike circle.
} 


\subsection{The group $S U(2,1)$ and its Lie algebra $\mathfrak{s u}(2,1)$}

The Lie group $S U(2,1)$ is defined as a subgroup of the group $G L(3, \mathbb{C})$ of invertible $(3 \times 3)$ complex matrices via

$$
S U(2,1)=\left\{g \in G L(3, \mathbb{C}): g^{\dagger} \eta g=\eta \text { and } \operatorname{det}(g)=1\right\} .
$$

Here, the defining metric $\eta$ is given by

$$
\eta=\left(\begin{array}{ccc}
0 & 0 & -i \\
0 & 1 & 0 \\
i & 0 & 0
\end{array}\right)
$$

and has signature $(++-)$. The condition $g^{\dagger} \eta g=\eta$ already implies $|\operatorname{det}(g)|$ $=1$ and so we can also think of $S U(2,1)$ as the set of unitary matrices $U(2,1)$ modulo a pure phase, $S U(2,1) \cong P U(2,1)$, with the projectivization $P$ referring to the equivalence relation $g \sim g e^{i \alpha}$ for $\alpha \in[0,2 \pi)$. The diagonal matrices $e^{i \alpha} \operatorname{diag}(1,1,1)$ form the center of the group $U(2,1)$.

The Lie group $S U(2,1)$ as defined in (2.1) has as Lie algebra of real dimension 8

$$
\mathfrak{s u}(2,1)=\left\{X \in \mathfrak{g l}(3, \mathbb{C}): X^{\dagger} \eta+\eta X=0 \text { and } \operatorname{tr}(X)=0\right\} .
$$

It consists of four compact and four non-compact generators, and the maximal real torus is one dimensional. We define the non-compact and compact Cartan generators

$$
H=\left(\begin{array}{ccc}
1 & 0 & 0 \\
0 & 0 & 0 \\
0 & 0 & -1
\end{array}\right), \quad J=\left(\begin{array}{ccc}
i & 0 & 0 \\
0 & -2 i & 0 \\
0 & 0 & i
\end{array}\right),
$$

the positive step operators

$$
\begin{aligned}
X_{1} & =\left(\begin{array}{ccc}
0 & -1+i & 0 \\
0 & 0 & 1-i \\
0 & 0 & 0
\end{array}\right), \quad \tilde{X}_{1}=\left(\begin{array}{ccc}
0 & 1+i & 0 \\
0 & 0 & 1+i \\
0 & 0 & 0
\end{array}\right), \\
X_{2} & =\left(\begin{array}{lll}
0 & 0 & 1 \\
0 & 0 & 0 \\
0 & 0 & 0
\end{array}\right),
\end{aligned}
$$


and the negative step operators

$$
\begin{aligned}
Y_{-1} & =\left(\begin{array}{ccc}
0 & 0 & 0 \\
1+i & 0 & 0 \\
0 & -1-i & 0
\end{array}\right), \quad \tilde{Y}_{-1}=\left(\begin{array}{ccc}
0 & 0 & 0 \\
-1+i & 0 & 0 \\
0 & -1+i & 0
\end{array}\right) \\
Y_{-2} & =\left(\begin{array}{ccc}
0 & 0 & 0 \\
0 & 0 & 0 \\
-1 & 0 & 0
\end{array}\right) .
\end{aligned}
$$

The subscript refers to the eigenvalue under the adjoint action of the noncompact Cartan generator $H$, e.g., $\left[H, X_{1}\right]=X_{1}$; the adjoint action of the compact Cartan generator $J$ is not diagonalizable over the real numbers. Furthermore, the generators satisfy

$$
\left[X_{1}, \tilde{X}_{1}\right]=-4 X_{2}
$$

such that the positive step operators form a Heisenberg algebra. Furthermore, the negative step operators $Y$ are minus the Hermitian conjugate of the positive step operators $X$.

The Lie algebra $\mathfrak{s u}(2,1)$ has a natural five grading by the generator $H$ as a direct sum of vector spaces

$$
\mathfrak{s u}(2,1)=\mathfrak{g}_{-2} \oplus \mathfrak{g}_{-1} \oplus \mathfrak{g}_{0} \oplus \mathfrak{g}_{1} \oplus \mathfrak{g}_{2},
$$

with

$$
\begin{aligned}
& \mathfrak{g}_{-2}=\mathbb{R} Y_{-2}, \quad \mathfrak{g}_{-1}=\mathbb{R} Y_{-1} \oplus \mathbb{R} \tilde{Y}_{-1}, \quad \mathfrak{g}_{0}=\mathbb{R} H \oplus \mathbb{R} J \\
& \mathfrak{g}_{1}=\mathbb{R} X_{1} \oplus \mathbb{R} \tilde{X}_{1}, \quad \mathfrak{g}_{2}=\mathbb{R} X_{2} .
\end{aligned}
$$

One sees that the $H$-eigenspaces with eigenvalue \pm 1 are degenerate. This is a characteristic feature of the reduced root system $B C_{1}$ underlying the real form $\mathfrak{s u}(2,1)$ of $\mathfrak{s l}(3, \mathbb{C})$. There is a single simple root $\alpha$ since the real rank of $\mathfrak{s u}(2,1)$ is one, and there are non-trivial root spaces $\mathfrak{g}_{1}$ and $\mathfrak{g}_{2}$ corresponding to $\alpha$ and $2 \alpha$, respectively. ${ }^{12}$ The $\mathfrak{s l}(2, \mathbb{R})$ subalgebra associated with the $2 \alpha$ root space is canonically normalized and can be given a standard basis for example with $H, E=X_{2}$ and $F=-Y_{-2}$, so that $[E, F]=H$. The corresponding $S L(2, \mathbb{R})$ subgroup of $S U(2,1)$ is given by matrices of

\footnotetext{
${ }^{12} \mathrm{~A}$ discussion of the restricted root system can, for example, be found in [67].
} 
the form

$$
\left\{\left(\begin{array}{lll}
a & 0 & b \\
0 & 1 & 0 \\
c & 0 & d
\end{array}\right): a, b, c, d \in \mathbb{R} \text { and } a d-b c=1\right\} \subset S U(2,1)
$$

Under this embedding, the fundamental representation of $S U(2,1)$ decomposes as $3=2 \oplus 1$. There exists a second, non-regular embedding of $S L(2, \mathbb{R})$ inside $S U(2,1)$, consisting of matrices of the form

$S L(2, \mathbb{R})$

$$
=\left\{\left(\begin{array}{ccc}
a^{2} & (-1+i) a b & i b^{2} \\
(-1-i) a c & a d+b c & (1-i) b d \\
-i c^{2} & (1+i) c d & d^{2}
\end{array}\right): a, b, c, d \in \mathbb{R} \text { and } a d-b c=1\right\} .
$$

Under this embedding, the fundamental representation of $S U(2,1)$ remains irreducible. The two subgroups (2.10) and (2.11) together generate the whole of $S U(2,1)$.

The Iwasawa decomposition of the Lie algebra $\mathfrak{s u}(2,1)$ reads

$$
\mathfrak{s u}(2,1)=\mathfrak{n}_{+} \oplus \mathfrak{a} \oplus \mathfrak{k},
$$

where the non-compact (Abelian) Cartan subalgebra is defined as $\mathfrak{a}=\mathbb{R} H$, while the nilpotent subspace $\mathfrak{n}_{+}=\mathbb{R} X_{1} \oplus \mathbb{R} \tilde{X}_{1} \oplus \mathbb{R} X_{2}$ is spanned by the positive step operators. The compact subalgebra of $\mathfrak{s u}(2,1)$ is $\mathfrak{k}=\mathfrak{s u}(2) \oplus$ $\mathfrak{u}(1)$ as a direct sum of Lie algebras. ${ }^{13}$ The generators of $\mathfrak{s u}(2)$ and $\mathfrak{u}(1)$ are given explicitly by the anti-Hermitian matrices

$$
\begin{aligned}
\hat{K}_{1} & =\frac{1}{4}\left(X_{1}+Y_{-1}\right), \quad \hat{K}_{2}=\frac{1}{4}\left(\tilde{X}_{1}+\tilde{Y}_{-1}\right), \quad \hat{K}_{3}=\frac{1}{4}\left(X_{2}+Y_{-2}+J\right), \\
\hat{J} & =\frac{3}{4}\left(X_{2}+Y_{-2}\right)-\frac{1}{4} J .
\end{aligned}
$$

\footnotetext{
${ }^{13}$ By contrast, the Iwasawa decomposition (2.12) is only a direct sum of vector spaces and not of Lie algebras.
} 
These satisfy $\left[\hat{J}, \hat{K}_{i}\right]=0$ and $\left[\hat{K}_{i}, \hat{K}_{j}\right]=-\epsilon_{i j k} \hat{K}_{k}$. The Weyl group of the reduced root system $B C_{1}$ is

$$
\mathcal{W}(\mathfrak{s u}(2,1))=\mathcal{W}\left(B C_{1}\right) \cong \mathbb{Z}_{2},
$$

corresponding to the Weyl reflection with respect to $\alpha$.

\subsection{Complex hyperbolic space}

The group $S U(2,1)$ acts transitively and isometrically on the complex twodimensional space ${ }^{14}$

$$
\mathbb{C H}^{2}=\left\{\mathcal{Z}=\left(z_{1}, z_{2}\right) \in \mathbb{C}^{2}: \mathcal{F}(\mathcal{Z})>0\right\},
$$

equipped with the Kähler metric

$$
d s^{2}=\frac{1}{4} \mathcal{F}^{-2}\left[d z_{1} d \bar{z}_{1}+i z_{2} d z_{1} d \bar{z}_{2}-i \bar{z}_{2} d z_{2} d \bar{z}_{1}+2 \Im\left(z_{1}\right) d z_{2} d \bar{z}_{2}\right] .
$$

The "height function" $\mathcal{F}: \mathbb{C}^{2} \rightarrow \mathbb{R}$ is defined by

$$
\mathcal{F}(\mathcal{Z}):=\Im\left(z_{1}\right)-\frac{1}{2}\left|z_{2}\right|^{2},
$$

and provides a Kähler potential for the metric (2.16),

$$
K_{\mathbb{C H}^{2}}(\mathcal{Z})=-\log \mathcal{F}(\mathcal{Z}) .
$$

The action of $S U(2,1)$ on $\mathcal{Z} \in \mathbb{C} \mathbb{H}^{2}$ is via fractional linear transformations

$$
g \cdot \mathcal{Z}=\frac{A \mathcal{Z}+B}{C \mathcal{Z}+D} \quad \text { for } \quad g=\left(\begin{array}{cc}
A & B \\
C & D
\end{array}\right),
$$

where the blocks $A, B, C$ and $D$ have the sizes $(2 \times 2),(2 \times 1),(1 \times 2)$ and $(1 \times 1)$, respectively, so that the denominator is a complex number. Since the height function transforms as

$$
\mathcal{F}(g \cdot \mathcal{Z})=\frac{\mathcal{F}(\mathcal{Z})}{|C \mathcal{Z}+D|^{2}}
$$

the condition $\mathcal{F}(\mathcal{Z})>0$ is preserved and the action is isometric. In fact, when verifying (2.20) one only requires the condition $g^{\dagger} \eta g=\eta$ so that (2.19)

\footnotetext{
${ }^{14}$ This is referred to as the "unbounded hyperquadric model" in [57].
} 
defines an action of all of $U(2,1)$ on complex hyperbolic two-space. Since elements from the center act trivially, one can restrict to $P U(2,1) \cong S U(2,1)$. We will refer to the space $\mathbb{C H}^{2}$ defined in (2.15) as the complex hyperbolic space, or the complex upper half plane. The slice $z_{2}=0, \Im\left(z_{1}\right)>0$ inside $\mathbb{C H}^{2}$ is preserved by the action of the $S L(2, \mathbb{R})$ subgroup in $(2.10)$, and gives an embedding of the standard Poincaré upper half plane inside $\mathbb{C H}^{2}$.

\subsection{Relation to the scalar coset manifold $S U(2,1) /(S U(2) \times U(1))$}

The complex hyperbolic upper half plane is isomorphic to the Hermitian symmetric space

$$
\mathbb{C} \mathbb{H}^{2} \cong S U(2,1) /(S U(2) \times U(1))
$$

where the right-hand side should properly be restricted to the connected component of the identity. This space can be parametrized by four real variables $\{\phi, \chi, \tilde{\chi}, \psi\}$, using the coset representative in the Iwasawa gauge (2.12),

$$
\begin{aligned}
\mathcal{V} & =e^{\chi X_{1}+\tilde{\chi} \tilde{X}_{1}+2 \psi X_{2}} e^{-\phi H} \\
& =\left(\begin{array}{ccc}
e^{-\phi} & \tilde{\chi}-\chi+i(\chi+\tilde{\chi}) & e^{\phi}\left(2 \psi+i\left(\chi^{2}+\tilde{\chi}^{2}\right)\right) \\
0 & 1 & e^{\phi}(\chi+\tilde{\chi}+i(\tilde{\chi}-\chi)) \\
0 & 0 & e^{\phi}
\end{array}\right) .
\end{aligned}
$$

The symmetric space is a right coset in our conventions, the coset element $\mathcal{V}$ transforming as $\mathcal{V} \rightarrow g \mathcal{V} k^{-1}$ with $g \in S U(2,1)$ and $k \in S U(2) \times U(1)$. The four scalar fields can take arbitrary real values.

It is convenient to define the Hermitian matrix

$$
\mathcal{K}=\mathcal{V} \mathcal{V}^{\dagger}
$$

that transforms as $\mathcal{K} \rightarrow g \mathcal{K} g^{\dagger}$ under the action of $g \in S U(2,1)$. Explicitly, this matrix reads

$$
\mathcal{K}=\left(\begin{array}{ccc}
e^{-2 \phi}+|\lambda|^{2}+e^{2 \phi}|\gamma|^{2} & i \bar{\lambda}+e^{2 \phi} \bar{\lambda} \gamma & e^{2 \phi} \gamma \\
-i \lambda+e^{2 \phi} \lambda \bar{\gamma} & 1+e^{2 \phi}|\lambda|^{2} & e^{2 \phi} \lambda \\
e^{2 \phi} \bar{\gamma} & e^{2 \phi} \bar{\lambda} & e^{2 \phi}
\end{array}\right)
$$

where, for later convenience, we have defined the complex variables

$$
\lambda:=\chi+\tilde{\chi}+i(\tilde{\chi}-\chi), \quad \gamma:=2 \psi+\frac{i}{2}|\lambda|^{2} .
$$


From $\mathcal{K}$ one can obtain the metric on the symmetric space via

$$
d s^{2}=-\frac{1}{8} \operatorname{tr}\left(d \mathcal{K} d\left(\mathcal{K}^{-1}\right)\right)=\frac{1}{8} \operatorname{tr}\left(\mathcal{V}^{-1} d \mathcal{V}+\left(\mathcal{V}^{-1} d \mathcal{V}\right)^{\dagger}\right)^{2}
$$

Working this out for the coset element (2.22) one finds the following $S U(2,1)$ invariant metric

$$
d s^{2}=d \phi^{2}+e^{2 \phi}\left(d \chi^{2}+d \tilde{\chi}^{2}\right)+e^{4 \phi}(d \psi+\chi d \tilde{\chi}-\tilde{\chi} d \chi)^{2} .
$$

Comparing (2.27) with (2.16) leads to the identification

$$
\begin{aligned}
& z_{1}=2 \psi+i\left(e^{-2 \phi}+\frac{1}{2}\left|z_{2}\right|^{2}\right)=2 \psi+i\left(e^{-2 \phi}+\chi^{2}+\tilde{\chi}^{2}\right), \\
& z_{2}=\chi+\tilde{\chi}+i(\tilde{\chi}-\chi) .
\end{aligned}
$$

Note that $z_{1}=\gamma+i e^{-2 \phi}, z_{2}=\lambda$, and the condition $0<\mathcal{F}(\mathcal{Z})=e^{-2 \phi}$ is automatically satisfied. Note that in terms of the real variables $\{\phi, \chi, \tilde{\chi}, \psi\}$ the Kähler potential (2.18) simply becomes $K_{\mathbb{C H}^{2}}=2 \phi$.

In the variables $\mathcal{Z}=\left(z_{1}, z_{2}\right)$ given by $(2.28)$, the matrix $\mathcal{K}$ of $(2.23)$ takes the simple form

$$
\mathcal{K}=\tilde{\mathcal{K}}+\eta,
$$

where $\eta$ is the defining matrix of $S U(2,1)$ given in $(2.2)$ and

$$
\tilde{\mathcal{K}}=e^{2 \phi}\left(\begin{array}{ccc}
\left|z_{1}\right|^{2} & z_{1} \bar{z}_{2} & z_{1} \\
\bar{z}_{1} z_{2} & \left|z_{2}\right|^{2} & z_{2} \\
\bar{z}_{1} & \bar{z}_{2} & 1
\end{array}\right)
$$

where one should bear in mind that $e^{2 \phi}=1 / \mathcal{F}(\mathcal{Z})$. The relations $(2.28)$ together with (2.19) allow one to determine the action of an element of $S U(2,1)$ in the real coordinates $\phi, \chi, \tilde{\chi}, \psi$. In particular, one may check that on the slice $\tilde{\chi}=\psi=0$, the $S L(2, \mathbb{R})$ subgroup $(2.11)$ acts by fractional linear transformations on the complex modulus $\chi+i e^{-\phi}$. This action may be considered as a remnant of the $S L(2, \mathbb{R})$ S-duality in 10-dimensional type IIB string theory. Similarly, on the slice $z_{2}=0$ the $S L(2, \mathbb{R})$ subgroup (2.10) acts by fractional linear transformations on the complex modulus $2 \psi+i e^{-2 \phi}$, which realizes four-dimensional S-duality. 


\subsection{Coset transformations and subgroups of $S U(2,1)$}

We now study the effect of some particular elements of $S U(2,1)$ on complex hyperbolic two-space, which have an immediate physical interpretation.

2.4.1. Heisenberg translations. Let $N$ denote the exponential of the nilpotent algebra of positive step operators $\mathfrak{n}_{+}$. We define the following elements of $N$ :

$$
T_{1}=\left(\begin{array}{ccc}
1 & -1+i & i \\
0 & 1 & 1-i \\
0 & 0 & 1
\end{array}\right), \quad \tilde{T}_{1}=\left(\begin{array}{ccc}
1 & 1+i & i \\
0 & 1 & 1+i \\
0 & 0 & 1
\end{array}\right), \quad T_{2}=\left(\begin{array}{ccc}
1 & 0 & 1 \\
0 & 1 & 0 \\
0 & 0 & 1
\end{array}\right)
$$

These are defined such that $T_{1}=\exp \left(X_{1}\right)$, etc. Any element $n \in N$ can be written as

$$
\begin{aligned}
n & =\left(T_{1}\right)^{a}\left(\tilde{T}_{1}\right)^{b}\left(T_{2}\right)^{c+2 a b}=e^{a X_{1}+b \tilde{X}_{1}+c X_{2}} \\
& =\left(\begin{array}{ccc}
1 & a(-1+i)+b(1+i) & c+i\left(a^{2}+b^{2}\right) \\
0 & 1 & a(1-i)+b(1+i) \\
0 & 0 & 1
\end{array}\right)
\end{aligned}
$$

for $a, b, c \in \mathbb{R}$. The effect of this transformation on $\mathcal{Z}=\left(z_{1}, z_{2}\right)$ is

$$
\begin{aligned}
& z_{1} \longmapsto z_{1}+[a(-1+i)+b(1+i)] z_{2}+c+i\left(a^{2}+b^{2}\right), \\
& z_{2} \longmapsto z_{2}+a(1-i)+b(1+i)
\end{aligned}
$$

or in terms of the four scalars fields of (2.22)

$$
\begin{aligned}
& \phi \longmapsto \phi, \\
& \chi \longmapsto \chi+a, \\
& \tilde{\chi} \longmapsto \tilde{\chi}+b, \\
& \psi \longmapsto \psi+\frac{1}{2} c-a \tilde{\chi}+b \chi .
\end{aligned}
$$

The appearance of the shift parameters $a$ and $b$ in the transformation of $\psi$ is due to the non-Abelian structure of $\mathfrak{n}_{+}$given by the Heisenberg algebra (2.7). This effect is also evident in the first line of expression (2.32) for the general element of $N$. From the point of view of the coset, the Heisenberg 
translations do not require any compensating transformation as they preserve the Iwasawa gauge.

2.4.2. Rotations. Rotations are generated by the compact Cartan element $J$ of $\mathfrak{s u}(2,1)$ given in $(2.4)$. Let

$$
R=\exp (\pi J / 2)=\left(\begin{array}{ccc}
i & 0 & 0 \\
0 & -1 & 0 \\
0 & 0 & i
\end{array}\right)
$$

then the most general transformation of this type is given by $R^{\sigma}:=e^{\sigma \pi J / 2}$, for $\sigma=0,1,2,3$, and acts on $\mathcal{Z}=\left(z_{1}, z_{2}\right)$ via

$$
z_{1} \rightarrow z_{1}, \quad z_{2} \rightarrow e^{i \pi \sigma / 2} z_{2} .
$$

In terms of the four scalar fields this transformation reads

$$
\begin{aligned}
& \phi \longmapsto \phi, \\
& \chi \longmapsto \cos (\pi \sigma / 2) \chi-\sin (\pi \sigma / 2) \tilde{\chi}, \\
& \tilde{\chi} \longmapsto \sin (\pi \sigma / 2) \chi+\cos (\pi \sigma / 2) \tilde{\chi}, \\
& \psi \longmapsto \psi
\end{aligned}
$$

and so rotates the two scalars $\chi$ and $\tilde{\chi}$ among each other while leaving the other two invariant. The compensating transformation to restore the Iwasawa gauge for the coset element (2.22) is $k=R^{\sigma}$.

2.4.3. Involution. The last transformation of interest is the involution

$$
S=\left(\begin{array}{ccc}
0 & 0 & i \\
0 & -1 & 0 \\
-i & 0 & 0
\end{array}\right)
$$

which acts on $\mathcal{Z}=\left(z_{1}, z_{2}\right)$ according to

$$
z_{1} \mapsto-\frac{1}{z_{1}}, \quad z_{2} \mapsto-i \frac{z_{2}}{z_{1}},
$$


corresponding to the non-trivial generator in the Weyl group (2.14). For the real scalars themselves we find the following transformation:

$$
\begin{aligned}
& \phi \longmapsto-\frac{1}{2} \ln \left[\frac{e^{-2 \phi}}{4 \psi^{2}+\left[e^{-2 \phi}+\chi^{2}+\tilde{\chi}^{2}\right]^{2}}\right], \\
& \chi \longmapsto \frac{2 \psi \tilde{\chi}-\left(e^{-2 \phi}+\chi^{2}+\tilde{\chi}^{2}\right) \chi}{4 \psi^{2}+\left[e^{-2 \phi}+\chi^{2}+\tilde{\chi}^{2}\right]^{2}}, \\
& \tilde{\chi} \longmapsto \frac{2 \psi \chi+\left(e^{-2 \phi}+\chi^{2}+\tilde{\chi}^{2}\right) \tilde{\chi}}{4 \psi^{2}+\left[e^{-2 \phi}+\chi^{2}+\tilde{\chi}^{2}\right]^{2}}, \\
& \psi \longmapsto-\frac{\psi}{4 \psi^{2}+\left[e^{-2 \phi}+\chi^{2}+\tilde{\chi}^{2}\right]^{2}} .
\end{aligned}
$$

It is straightforward to check that the required compensating transformation in this case indeed belongs to the maximal compact subgroup $S U(2) \times U(1)$.

\subsection{The Picard modular group}

We finally discuss the Picard modular group $S U(2,1 ; \mathbb{Z}[i])$. This group can be defined as the intersection [57]

$$
S U(2,1 ; \mathbb{Z}[i]):=S U(2,1) \cap S L(3, \mathbb{Z}[i]),
$$

where $\mathbb{Z}[i]$ denotes the Gaussian integers

$$
\mathbb{Z}[i]=\{z \in \mathbb{C}: \Re(z), \Im(z) \in \mathbb{Z}\}=\left\{m_{1}+i m_{2}: m_{1}, m_{2} \in \mathbb{Z}\right\}
$$

This definition implies that any element $g \in S U(2,1)$ which has only Gaussian integer matrix entries belongs to $S U(2,1 ; \mathbb{Z}[i])$. In view of the discussion of $P U(2,1) \cong S U(2,1)$ the Picard modular group can also be called $P U(2,1 ; \mathbb{Z}[i])$.

Let us now examine the particular $S U(2,1)$-transformations of the previous subsection to check whether they belong to the Picard group. The Heisenberg group $N \subset S U(2,1)$ contains a subgroup $N(\mathbb{Z}):=N \cap$ $S U(2,1 ; \mathbb{Z}[i])$. By inspection of Equation $(2.32)$ we see that $N(\mathbb{Z})$ must be of the form

$$
N(\mathbb{Z})=\left\{e^{a X_{1}+b \tilde{X}_{1}+c X_{2}}: a, b, c \in \mathbb{Z}\right\}
$$

In view of $(2.32)$, a natural set of generators for $N(\mathbb{Z})$ is given by the three matrices in (2.31) $T_{1}, \tilde{T}_{1}$ and $T_{2}$. The action of these discrete shifts are then 
as given in (2.34) with parameters $a, b, c \in \mathbb{Z}$. The translations (2.31) are of infinite order in the Picard modular group.

The rotation $R$ defined in (2.35) is an element of order 4 in the Picard modular group, and $R^{\sigma}$ belongs to $S U(2,1 ; \mathbb{Z}[i])$ for the discrete values of the exponent $\sigma=0,1,2,3$. The action of $R$ on the scalar fields is

$$
R: \quad(\chi, \tilde{\chi}) \longmapsto(-\tilde{\chi}, \chi),
$$

while leaving $\phi$ and $\psi$ invariant. Physically speaking, this corresponds to electric-magnetic duality, which is expected to be preserved in the quantum theory [47].

Finally, we will examine the involution $S$ in Equation (2.38). Clearly, this involution is an element (of order 2) in the Picard modular group. As already noted above, the involution (2.38) corresponds to the Weyl reflection of the restricted root system $B C_{1}$ of the non-split real form $\mathfrak{s u}(2,1)$. This reflection is associated with the long root $2 \alpha$. In this context, we can also give an interpretation of the rotation $R$. This is a transformation that rotates within the degenerate, two-dimensional $\alpha$ root space, spanned by the generators $X_{1}$ and $\tilde{X}_{1}$.

The Picard modular group acts discontinuously on the complex hyperbolic space $\mathbb{C H}^{2}$. A fundamental domain for its action has been given by Francsics and Lax in [57]. Recently, together with Falbel and Parker, they have also proven that the Picard modular group $S U(2,1 ; \mathbb{Z}[i])$ is generated by the translations $T_{1}$ and $T_{2}$, together with the rotation $R$ and the involution $S[56]^{15}$

Since the two translations $T_{1}$ and $\tilde{T}_{1}$ are related through "electricmagnetic duality" by $\tilde{T}_{1}=R T_{1} R^{-1}$, one may equivalently choose either of the translations $T_{1}$ or $\tilde{T}_{1}$ associated with the $\alpha$ root space in the theorem. Since all three translations $T_{1}, \tilde{T}_{1}$ and $T_{2}$ will turn out to have a clear physical interpretation, we present the Picard modular group as generated (non-minimally) by the following five elements:

$$
T_{1}=\left(\begin{array}{ccc}
1 & -1+i & i \\
0 & 1 & 1-i \\
0 & 0 & 1
\end{array}\right), \quad \tilde{T}_{1}=\left(\begin{array}{ccc}
1 & 1+i & i \\
0 & 1 & 1+i \\
0 & 0 & 1
\end{array}\right), \quad T_{2}=\left(\begin{array}{ccc}
1 & 0 & 1 \\
0 & 1 & 0 \\
0 & 0 & 1
\end{array}\right)
$$

$$
R=\left(\begin{array}{ccc}
i & 0 & 0 \\
0 & -1 & 0 \\
0 & 0 & i
\end{array}\right), \quad S=\left(\begin{array}{ccc}
0 & 0 & i \\
0 & -1 & 0 \\
-i & 0 & 0
\end{array}\right)
$$

\footnotetext{
${ }^{15}$ We are very grateful to G. Francsics and P. Lax for communicating this result to us prior to publication.
} 


\section{Eisenstein series for the Picard modular group}

In this section we shall construct Eisenstein series for the Picard modular group in the principal continuous series representation of $S U(2,1)$. We shall give three different constructions, which, despite being equivalent, mutually enlighten each other. In Section 3.1 we construct a manifestly $S U(2,1 ; \mathbb{Z}[i])$-invariant function on $S U(2,1) /(S U(2) \times U(1))$ by summing over points in the three-dimensional Gaussian lattice $\mathbb{Z}[i]^{3}$. This produces a non-holomorphic Eisenstein series $\mathcal{E}_{s}$, parametrized by $s$, which will be the central object of study in the remainder of this paper. In Section 3.2, we use the isomorphism between the coset space $S U(2,1) /(S U(2) \times U(1))$ and the complex upper half plane $\mathbb{C} \mathbb{H}^{2}$ to construct a Poincaré series $\mathcal{P}_{s}$ on $\mathbb{C H}^{2}$. This turns out to be identical to $\mathcal{E}_{s}$ up to an $s$-dependent Dedekind zeta function factor. For completeness, in Appendix B we give a third construction using standard adelic techniques, which illuminates the representation-theoretic nature of $\mathcal{E}_{s}$.

\subsection{Lattice construction and quadratic constraint}

Following [13], a non-holomorphic function on the double quotient

$$
S U(2,1 ; \mathbb{Z}[i]) \backslash S U(2,1) /(S U(2) \times U(1))
$$

can be constructed as the Eisenstein series ${ }^{16}$

$$
\begin{aligned}
& \mathcal{E}_{s}(\mathcal{K}):=\sum_{\substack{\vec{\omega} \in\left[i[]^{3} \\
\vec{\omega} \dagger \cdot \eta \vec{\omega}=0\right.}}^{\prime}\left[\vec{\omega}^{\dagger} \cdot \mathcal{K} \cdot \vec{\omega}\right]^{-s} \\
& (3.2)=\sum_{\substack{\vec{\omega} \in \mathbb{Z}[i]]^{3} \\
\tilde{\omega}^{\dagger} \cdot \eta \cdot \vec{\omega}=0}}^{\prime} e^{-2 s \phi}\left[\left|\omega_{1}+\omega_{2} \lambda+\omega_{3} \gamma\right|^{2}+e^{-2 \phi}\left|\omega_{2}+i \omega_{3} \bar{\lambda}\right|^{2}+e^{-4 \phi}\left|\omega_{3}\right|^{2}\right]^{-s},
\end{aligned}
$$

where $\mathcal{K}=\mathcal{V} \mathcal{V}^{\dagger}$ is the "generalized metric" (2.24), and the variables $\lambda$ and $\gamma$ were defined as functions of $\mathcal{Z}=\left(z_{1}, z_{2}\right)$ in (2.25). In (3.2) the sum runs over 3 -vectors of Gaussian integers $\vec{\omega} \neq(0,0,0)$ subject to the quadratic constraint

$$
\vec{\omega}^{\dagger} \cdot \eta \cdot \vec{\omega}=\left|\omega_{2}\right|^{2}-2 \Im\left(\omega_{1} \bar{\omega}_{3}\right)=0, \quad \vec{\omega}:=\left(\begin{array}{c}
\bar{\omega}_{3} \\
\bar{\omega}_{2} \\
\bar{\omega}_{1}
\end{array}\right) .
$$

${ }^{16}$ We note that the same summand and constraint appear in the analysis of [68]. 
Setting

$$
\omega_{1}=m_{1}+i m_{2}, \quad \omega_{2}=n_{1}+i n_{2}, \quad \omega_{3}=p_{1}+i p_{2},
$$

this may be rewritten as a sum over six integers $m_{i}, n_{i}, p_{i}$, not all vanishing, subject to the constraint

$$
\vec{\omega}^{\dagger} \cdot \eta \cdot \vec{\omega}=n_{1}^{2}+n_{2}^{2}+2 m_{1} p_{2}-2 m_{2} p_{1}=0
$$

The Eisenstein series defined in (3.2) converges absolutely for $\Re(s)>2$.

To explain the role of the quadratic constraint (3.3), it is convenient to utilize the isomorphism between the coset space $S U(2,1) /(S U(2) \times U(1))$ and the complex hyperbolic space $\mathbb{C H}^{2}$, as discussed in Section 2.3. We recall from (2.30) that in terms of the variable $\mathcal{Z}=\left(z_{1}, z_{2}\right) \in \mathbb{C H}^{2}$, the matrix $\mathcal{K}$ reads

$$
\mathcal{K}=\tilde{\mathcal{K}}+\eta
$$

where $\eta$ is the $S U(2,1)$-invariant metric, Equation $(2.2)$, and the matrix $\tilde{\mathcal{K}}$ is given by

$$
\tilde{\mathcal{K}}=e^{2 \phi}\left(\begin{array}{ccc}
\left|z_{1}\right|^{2} & z_{1} \bar{z}_{2} & z_{1} \\
\bar{z}_{1} z_{2} & \left|z_{2}\right|^{2} & z_{2} \\
\bar{z}_{1} & \bar{z}_{2} & 1
\end{array}\right)=\tilde{\mathcal{V}} \tilde{\mathcal{V}}^{\dagger} \quad \text { for } \quad \tilde{\mathcal{V}}=e^{\phi}\left(\begin{array}{ccc}
0 & 0 & z_{1} \\
0 & 0 & z_{2} \\
0 & 0 & 1
\end{array}\right) \text {. }
$$

In this new parametrization, the Eisenstein series becomes

$$
\begin{aligned}
& \mathcal{E}_{s}(\mathcal{Z})=\sum_{\substack{\vec{\omega} \in \mathbb{Z}[i]^{3} \\
\vec{\omega}^{\dagger} \cdot \eta \cdot \vec{\omega}=0}}^{\prime}\left[\vec{\omega}^{\dagger} \cdot \tilde{\mathcal{K}} \cdot \vec{\omega}+\vec{\omega}^{\dagger} \cdot \eta \cdot \vec{\omega}\right]^{-s} \\
& =\sum_{\substack{\omega \in \mathbb{Z}[i]^{3} \\
\omega^{\dagger} \cdot \eta \cdot \vec{\omega}=0}}^{\prime} e^{-2 s \phi}\left|\omega_{1}+\omega_{2} z_{2}+\omega_{3} z_{1}\right|^{-2 s} .
\end{aligned}
$$

The constraint (3.3) can now be motivated as follows [13]. Since the coset representative $\mathcal{V} \in S U(2,1) /(S U(2) \times U(1))$ transforms in the fundamental representation $\mathcal{R}$ of $S U(2,1)$, the generalized metric $\mathcal{K}=\mathcal{V} \mathcal{V}^{\dagger}$ transforms in the symmetric tensor product $\mathcal{R} \otimes_{s} \mathcal{R}$. As reflected in (3.6), this tensor product is not irreducible. In order for $\mathcal{E}_{s}$ to be an eigenfunction of the Laplacian on $\mathbb{C H}^{2}$, it is necessary to project out the singlet component in (3.6), hence to enforce the constraint (3.3) in the sum. To be specific, the 
Laplacian on the coset space $\mathbb{C H}^{2}$, written in terms of the real variables $\left\{y=e^{-2 \phi}, \chi, \tilde{\chi}, \psi\right\}$, is given by

$$
\begin{aligned}
\Delta_{\mathbb{C H}^{2}}= & \frac{1}{4} y\left(\partial_{\chi}^{2}+\partial_{\tilde{\chi}}^{2}\right)+\frac{1}{4}\left(y^{2}+y\left(\chi^{2}+\tilde{\chi}^{2}\right)\right) \partial_{\psi}^{2} \\
& +\frac{1}{2} y\left(\tilde{\chi} \partial_{\chi}-\chi \partial_{\tilde{\chi}}\right) \partial_{\psi}+y^{2} \partial_{y}^{2}-y \partial_{y} .
\end{aligned}
$$

Taking into account the quadratic constraint (3.3), it is straightforward to check that $\mathcal{E}_{s}$ is an eigenvector of the Laplacian with eigenvalue $s(s-2)$, as stated in (1.13) above.

Since $S U(2,1)$ admits two Casimir operators of degree 2 and 3 , and since $\Delta_{\mathbb{C H}^{2}}$ represents the action of the quadratic Casimir on the space of (squareintegrable) functions on $S U(2,1) /(S U(2) \times U(1))$, one may ask whether $\mathcal{E}_{s}$ is also an eigenvector of an invariant differential operator of degree 3 . It turns out however, as already noticed in [63], that the principal representation of the cubic Casimir in the space of functions on $S U(2,1) /(S U(2) \times U(1))$ vanishes identically. In terms of the parametrization of the Casimir eigenvalues by the complex variables $(p, q)$ used in $[63,69]$, the Eisenstein series $\mathcal{E}_{s}$ is attached to the principal spherical representation with $p=q=s-2$ (see Appendix B for some details on the principal series of $S U(2,1))$.

Let us also comment on the functional dimension of the representation associated to $\mathcal{E}_{s}$. The summation ranges over six (real) integers coordinating the lattice $\mathbb{Z}[i]^{3} \sim \mathbb{Z}^{6}$. Since both the summand and the constraint are homogeneous in $\vec{\omega}$ one can factor out an overall common Gaussian integer. Among the remaining four real integers the (real) quadratic constraint $\left|\omega_{2}\right|^{2}-2 \Im\left(\omega_{1} \bar{\omega}_{3}\right)=0$ eliminates one of the summation variables, leaving effectively a sum over three integers only. This is consistent with the functional dimension 3 of the principal continuous series representation of $S U(2,1)$ and the number of expected different instanton contributions.

\subsection{Poincaré series on the complex upper half plane}

In the mathematical literature, a standard way of constructing non-holomorphic Eisenstein series on a symmetric space $G / K$ is in terms of Poincaré series. For the case of the coset space $S L(2, \mathbb{R}) / S O(2)$, parametrized by a complex coordinate $\tau$, such a Poincaré series is obtained by summing the function $\Im(\gamma \cdot \tau)^{s}$ over the orbit $\gamma \in \Gamma_{\infty} \backslash S L(2, \mathbb{Z})$, where $\Gamma_{\infty}$ is generated by $T: \tau \mapsto \tau+1$. This indeed produces a non-holomorphic Eisenstein series on the double quotient $S L(2, \mathbb{Z}) \backslash S L(2, \mathbb{R}) / S O(2)$ with eigenvalue $s(s-1)$ under the Laplacian on $S L(2, \mathbb{R}) / S O(2)$ (for a very nice treatment, see [70]). 
Here we generalize this construction to the case of the complex upper half plane $\mathbb{C H} \mathbb{H}^{2}$, parametrized by the variable $\mathcal{Z}=\left(z_{1}, z_{2}\right)$. The generalization of $\Im(\tau)$ is then given by the $N(\mathbb{Z})$-invariant function $\mathcal{F}(\mathcal{Z})$, constructed in (2.17) [71]. ${ }^{17}$ The invariance of $\mathcal{F}(\mathcal{Z})$ under $N(\mathbb{Z})$ can be checked by direct substitution of the Heisenberg translations in Equation (2.33). As we have seen in Section 2, the Picard modular group $S U(2,1 ; \mathbb{Z}[i])$ acts by fractional transformations on $\mathcal{Z} \in \mathbb{C H}^{2}$ such that the function $\mathcal{F}(\mathcal{Z})$ transforms as

$$
\mathcal{F}(\gamma \cdot \mathcal{Z})=\frac{\mathcal{F}(\mathcal{Z})}{|C \mathcal{Z}+D|^{2}}, \quad \gamma=\left(\begin{array}{cc}
A & B \\
C & D
\end{array}\right) \in S U(2,1 ; \mathbb{Z}[i]) .
$$

A Poincaré series for the Picard group may now be constructed as follows:

$$
\mathcal{P}_{s}(\mathcal{Z}):=\sum_{\gamma \in N(\mathbb{Z}) \backslash S U(2,1 ; \mathbb{Z}[i])} \mathcal{F}(\gamma \cdot \mathcal{Z})^{s}=\sum_{\gamma \in N(\mathbb{Z}) \backslash S U(2,1 ; \mathbb{Z}[i])}\left(\frac{\mathcal{F}(\mathcal{Z})}{|C \mathcal{Z}+D|^{2}}\right)^{s} .
$$

Taking $C:=\left(\omega_{3}, \omega_{2}\right) \in \mathbb{Z}[i]^{2}$ and $D:=\omega_{1} \in \mathbb{Z}[i]$, and recalling that $\mathcal{F}(\mathcal{Z})=$ $e^{-2 \phi}$, then reproduces the same form of the Eisenstein series as in Equation (3.8), i.e.,

$$
\mathcal{P}_{s}(\mathcal{Z})=\sum_{\gamma \in N(\mathbb{Z}) \backslash S U(2,1 ; \mathbb{Z}[i])} e^{-2 s \phi}\left|\omega_{1}+\omega_{2} z_{2}+\omega_{3} z_{1}\right|^{-2 s} .
$$

The sum over orbits in $N(\mathbb{Z}) \backslash S U(2,1 ; \mathbb{Z}[i])$ is equivalent to the sum over the Gaussian lattice $\mathbb{Z}[i]^{3}$ modulo the constraint $\vec{\omega}^{\dagger} \cdot \eta \cdot \vec{\omega}=0$, together with a coprime condition on the summation variables $\vec{\omega}[66]$ :

$$
\mathcal{P}_{s}(\mathcal{Z})=\sum_{\substack{\vec{\omega} \in \mathbb{Z}[i]^{3}, \operatorname{gcd}\left(\omega_{1}^{\prime}, \omega_{2}^{\prime}, \omega_{3}^{\prime}\right)=1 \\ \vec{\omega}^{\prime \dagger} \cdot \vec{\omega}^{\prime}=0}}^{\prime} e^{-2 s \phi}\left|\omega_{1}^{\prime}+\omega_{2}^{\prime} z_{2}+\omega_{3}^{\prime} z_{1}\right|^{-2 s} .
$$

Defining $\vec{\omega}=\vec{\omega}^{\prime} \beta$ with $\beta=\operatorname{gcd}\left(\omega_{1}, \omega_{2}, \omega_{3}\right) \in \mathbb{Z}[i]$ and inserting this into (3.2) we then have the relation

$$
\mathcal{E}_{s}(\phi, \lambda, \gamma)=4 \zeta_{\mathbb{Q}(i)}(s) \mathcal{P}_{s}(\mathcal{Z}),
$$

where $\zeta_{\mathbb{Q}(i)}(s)$ is the Dedekind zeta function for the quadratic extension $\mathbb{Q}(i)$ of the rational numbers, and the overall factor of 4 originates from the four units in $\mathbb{Z}[i]$. This will be discussed in more detail in Section 4.2 (see Equation (4.15)).

\footnotetext{
${ }^{17}$ We are grateful to Genkai Zhang for helpful discussions on this construction.
} 


\section{Fourier expansion of $\mathcal{E}_{s}(\phi, \chi, \tilde{\chi}, \psi)$}

In this section we compute the Fourier expansion of the Eisenstein series (3.2). We begin by recalling the general decomposition with respect to the action of the Heisenberg subgroup $N \subset S U(2,1)$.

\subsection{General structure of the non-Abelian Fourier expansion}

The main complication of the Fourier expansion stems from the non-Abelian nature of the nilpotent group $N \subset S U(2,1) . N$ is isomorphic to a threedimensional Heisenberg group, where the center $Z=[N, N]$ is parametrized by $\psi$. The Fourier expansion therefore splits into an Abelian part and a nonAbelian part. The Abelian term corresponds to an expansion with respect to the abelianized group $N / Z$, while the non-Abelian terms represent the expansion with respect to the center $Z$. This general structure of the Fourier expansion of automorphic forms for the Picard modular group is discussed in detail by Ishikawa [64], to which we refer the interested reader. A similar discussion may also be found in the mathematics [72,73] and physics [34] literature for the case of automorphic forms on $S L(3, \mathbb{R}) / S O(3)$.

We have seen in Section 2 that the action of an arbitrary Heisenberg shift $\mathcal{U}_{a, b ; c} \in N(\mathbb{Z})=N \cap S U(2,1 ; \mathbb{Z}[i])$ on $\chi, \tilde{\chi}$ and $\psi$ is given by

$$
\begin{aligned}
\mathcal{U}_{a, b ; c}: \chi & \longmapsto \chi+a \\
\tilde{\chi} & \longmapsto \tilde{\chi}+b \\
\psi & \longmapsto \psi+\frac{1}{2} c-a \tilde{\chi}+b \chi
\end{aligned}
$$

for $a, b, c \in \mathbb{Z}$. Since the Eisenstein series (3.2) is in particular invariant under $N(\mathbb{Z})$ we can organize the Fourier expansion by diagonalizing different subgroups of the non-Abelian Heisenberg group $N(\mathbb{Z})$.

Explicitly, we write the general form of the Fourier expansion as

$$
\mathcal{E}_{s}(\phi, \chi, \tilde{\chi}, \psi)=\mathcal{E}_{s}^{(\mathrm{const})}(\phi)+\mathcal{E}_{s}^{(\mathrm{A})}(\phi, \chi, \tilde{\chi})+\mathcal{E}_{s}^{(\mathrm{NA})}(\phi, \chi, \tilde{\chi}, \psi),
$$

where $\mathcal{E}_{s}^{(\text {const })}(\phi)$ is the constant term and

$$
\begin{aligned}
\mathcal{E}_{s}^{(\mathrm{A})}(\phi, \chi, \tilde{\chi}) & =\sum_{\left(\ell_{1}, \ell_{2}\right) \in \mathbb{Z}^{2}}^{\prime} \mathfrak{C}_{\ell_{1}, \ell_{2}}^{(\mathrm{A})}(\phi ; s) e^{-2 \pi i\left(\ell_{1} \chi+\ell_{2} \tilde{\chi}\right)}, \\
\mathcal{E}_{s}^{(\mathrm{NA})}(\phi, \chi, \tilde{\chi}, \psi) & =\sum_{k \in \mathbb{Z}}^{\prime} \mathfrak{C}_{k}^{(\mathrm{NA})}(\phi, \chi, \tilde{\chi} ; s) e^{-4 \pi i k \psi}
\end{aligned}
$$


are called the Abelian and non-Abelian terms, respectively. Following [34, 64 ], we proceed to extract an additional phase factor in the non-Abelian term, which accounts for the shifts of $\psi$ along the non-central directions. This yields the following structure of the non-Abelian term:

$$
\mathcal{E}_{s}^{(\mathrm{NA})}(\phi, \chi, \tilde{\chi}, \psi)=\sum_{k \in \mathbb{Z}}^{\prime} \sum_{\ell=0}^{4|k|-1} \sum_{n \in \mathbb{Z}+\frac{\ell}{4|k|}} \mathfrak{C}_{k, \ell}^{(\mathrm{NA})}(\phi, \tilde{\chi}-n ; s) e^{8 \pi i k n \chi-4 \pi i k(\psi+\chi \tilde{\chi})} .
$$

The Abelian term is manifestly invariant under shifts of the form $\mathcal{U}_{a, b ; 0} \in$ $N(\mathbb{Z}) / Z$. For the non-Abelian term, invariance under

$$
\begin{aligned}
\mathcal{U}_{1,0 ; 0}: \chi & \longmapsto \chi+1 \\
: \psi & \longmapsto \psi-\tilde{\chi}
\end{aligned}
$$

is manifest since $4 k n \in \mathbb{Z}$. On the other hand, the transformation

$$
\begin{aligned}
\mathcal{U}_{0,1 ; 0}: \tilde{\chi} & \longmapsto \tilde{\chi}+1 \\
: \psi & \longmapsto \psi+\chi
\end{aligned}
$$

requires a compensating shift $n \mapsto n+1$ on the summation, under which the variation of the total phase cancels. Note also the restricted dependence on $\tilde{\chi}$ in the Fourier coefficient; upon shifting $\tilde{\chi} \mapsto \tilde{\chi}+1$ and compensating $n \mapsto n+1$ the coefficient is indeed invariant. Finally, invariance under $\mathcal{U}_{0,0 ; 1}$ is manifest since this gives an overall phase $e^{-4 \pi i k / 2}=1$.

Note that in writing the non-Abelian term (4.4) we have made an explicit choice of polarization, in the sense that we have manifestly diagonalized the action of Heisenberg shifts of the restricted form $\mathcal{U}_{a, 0 ; c}$. We could have chosen the opposite polarization in which we instead diagonalize the action of $\mathcal{U}_{0, b ; c}$. In this case, the non-Abelian term reads

$$
\mathcal{E}_{s}^{(\mathrm{NA})}(\phi, \chi, \tilde{\chi}, \psi)=\sum_{k \in \mathbb{Z}}^{\prime} \sum_{\ell^{\prime}=0}^{4|k|-1} \sum_{\tilde{n} \in \mathbb{Z}+\frac{\ell^{\prime}}{4|k|}} \tilde{\mathfrak{C}}_{k, \ell^{\prime}}^{(\mathrm{NA})}(\phi, \chi-\tilde{n} ; s) e^{-8 \pi i k \tilde{n} \tilde{\chi}-4 \pi i k(\psi-\chi \tilde{\chi})}
$$

The Fourier coefficients $\mathfrak{C}_{k, \ell}^{(\mathrm{NA})}$ and $\tilde{\mathfrak{C}}_{k, \ell^{\prime}}^{(\mathrm{NA})}$ in the two different polarizations are related via a Fourier transform (see [34]). In the sequel we work for definiteness with the first polarization defined by (4.4). 
Besides invariance under the Heisenberg group we can also use invariance under the electric-magnetic duality transformation $R:(\chi, \tilde{\chi}) \mapsto(-\tilde{\chi}, \chi)$ of (2.44). On the Abelian term this implies that the coefficient $\left.\mathfrak{C}_{\ell_{1}, \ell_{2}} \mathrm{~A}\right)$ is invariant under $\pi / 2$ rotations of $\left(\ell_{1}, \ell_{2}\right)$. On the non-Abelian term (4.4) application of $R$ leads to

$$
\mathcal{E}_{s}^{(\mathrm{NA})}(\phi, \chi, \tilde{\chi}, \psi)=\sum_{k \in \mathbb{Z}}^{\prime} \sum_{\ell=0}^{4|k|-1} \sum_{n \in \mathbb{Z}+\frac{\ell}{4|k|}} \mathfrak{C}_{k, \ell}^{(\mathrm{NA})}(\phi, \chi-n ; s) e^{-8 \pi i k n \tilde{\chi}-4 \pi i k(\psi-\chi \tilde{\chi})}
$$

and hence we have $\mathfrak{C}_{k, \ell}^{(\mathrm{NA})}=\tilde{\mathfrak{C}}_{k, \ell}^{(\mathrm{NA})}$, relating the two choices of polarization as to be expected from electric-magnetic duality. Applying $R$ again leads to relations among the coefficients $\mathfrak{C}_{k, \ell}^{(\mathrm{NA})}$ and $\mathfrak{C}_{k, \ell^{\prime}}^{(\mathrm{NA})}$ for different $\ell$ and $\ell^{\prime}$.

Finally, we can use the Laplacian condition on the Eisenstein series $\mathcal{E}_{s}$ (see Equation (1.13)) to further constrain the Fourier coefficients $\mathfrak{C}_{\ell_{1}, \ell_{2}}^{(\mathrm{A})}$ and $\mathfrak{C}_{k, \ell}^{(\mathrm{NA})}$ and determine their functional dependence on the moduli. In all cases, we require normalizability of the solution, which physically means a wellbehaved "weak-coupling" limit $e^{\phi} \rightarrow 0$. Plugging in the Abelian term $\mathcal{E}_{s}^{(\mathrm{A})}$ into the eigenvalue equation (1.13) yields an equation for the $\phi$-dependence of the coefficients, which is solved by a modified Bessel function. More precisely, we find that the Abelian term in the expansion takes the form

$$
\begin{aligned}
\mathcal{E}_{s}^{(\mathrm{A})}(\phi, \chi, \tilde{\chi}, \psi)= & e^{-2 \phi} \sum_{\left(\ell_{1}, \ell_{2}\right) \in \mathbb{Z}^{2}}^{\prime} C_{\ell_{1}, \ell_{2}}^{(\mathrm{A})}(s) \\
& \times K_{2 s-2}\left(2 \pi e^{-\phi} \sqrt{\ell_{1}^{2}+\ell_{2}^{2}}\right) e^{-2 \pi i\left(\ell_{1} \chi+\ell_{2} \tilde{\chi}\right)},
\end{aligned}
$$

where the remaining coefficients $C_{\ell_{1}, \ell_{2}}^{(\mathrm{A})}(s)$ are now independent of $\phi$ and encode the arithmetic information of the group $S U(2,1 ; \mathbb{Z}[i])$. The precise form of these numerical coefficients will be computed in Section 4.5 below.

Turning to the non-Abelian term (4.4), the Laplacian condition on the coefficient separates into a harmonic oscillator equation in the variable $x=$ $\tilde{\chi}-n$, with solution given by a Hermite polynomial $H$, as well as a hypergeometric equation in the variable $y=e^{-2 \phi}$ whose solution can be written in terms of a Whittaker function $W$. The separation of variables induces a sum over the eigenvalues of the harmonic oscillator, leading to the following 
structure for the non-Abelian term:

$$
\begin{aligned}
\mathcal{E}_{s}^{(\mathrm{NA})}(\phi, \chi, \tilde{\chi}, \psi)= & e^{-\phi} \sum_{k \in \mathbb{Z}}^{\prime} \sum_{\ell=0}^{4|k|-1} \sum_{n \in \mathbb{Z}+\frac{\ell}{4|k|}} \sum_{r=0}^{\infty} C_{r, k, \ell}^{(\mathrm{NA})}(s)|k|^{1 / 2-s} e^{-4 \pi|k|(\tilde{\chi}-n)^{2}} \\
& \times H_{r}(\sqrt{8 \pi|k|}(\tilde{\chi}-n)) W_{-r-\frac{1}{2}, s-1}\left(4 \pi|k| e^{-2 \phi}\right) \\
& \times e^{8 \pi i k n \chi-4 \pi i k(\psi+\chi \tilde{\chi})},
\end{aligned}
$$

where the numerical coefficients $C_{r, k, \ell}^{(\mathrm{NA})}(s)$ will be further discussed in Section 4.6 .

We shall now proceed to compute the explicit form of the Fourier expansion; that is, determine the constant term $\mathcal{E}_{s}^{\text {(const) }}$ as well as the Abelian and non-Abelian numerical Fourier coefficients $C_{\ell_{1}, \ell_{2}}^{(\mathrm{A})}(s)$ and $C_{r, k, \ell}^{(\mathrm{NA})}(s)$.

\subsection{First constant term}

The constant term ${ }^{18}$ is defined generally as

$$
\mathcal{E}_{s}^{\text {(const) }}(\phi)=\int_{0}^{1} d \chi \int_{0}^{1} d \tilde{\chi} \int_{0}^{1 / 2} d \psi \mathcal{E}_{s}(\phi, \chi, \tilde{\chi}, \psi),
$$

where the integral over the coordinate $\psi$ (physically, the NS-axion modulus) runs from 0 to $1 / 2$ because of the extra factor of 2 in front of $\psi$ in our parametrization of $N$ in Equation (2.22). Since the Cartan subgroup $A$ appearing in the Iwasawa decomposition of $S U(2,1)$ is one-dimensional, the constant term only depends on the dilatonic scalar $\phi$. Moreover, recall from the discussion in Section 2.4 that the Weyl group of $\mathfrak{s u}(2,1)$ is the Weyl group of the restricted root system $B C_{1}$, which is isomorphic with $\mathbb{Z}_{2}$. Hence, the constant term $\mathcal{E}_{s}^{(\text {const })}(\phi)$ consists of two contributions, $\mathcal{E}_{s}^{(0)}$ and $\mathcal{E}_{s}^{(1)}$, which are permuted by $\mathbb{Z}_{2}[65] .{ }^{19}$

The powers of $e^{\phi}$ in $\mathcal{E}_{s}^{\text {(const) }}(\phi)$ may be determined by the Laplacian condition on $\mathcal{E}_{s}$. In Section 3.2 we have seen that the Eisenstein series is an eigenfunction of the Laplacian $\Delta_{\mathbb{C H}}{ }^{2}$ with eigenvalue $s(s-2)$. This implies that all the constant terms must individually be eigenfunctions of $\Delta_{\mathbb{C H}}$ with

\footnotetext{
${ }^{18}$ The terminology constant term is derived from holomorphic Eisenstein series where these terms are truly constant and independent of the scalar fields. For nonholomorphic Eisenstein series, as the one studied here, the constant terms retain a dependence on the fields corresponding to Cartan generators.

${ }^{19}$ We are grateful to Pierre Vanhove for helpful discussions on the constant terms.
} 
the same eigenvalue. It turns out that there is a unique solution to this, and we find that $\mathcal{E}_{s}^{(0)}$ must be of the form

$$
\mathcal{E}_{s}^{(\text {const })}(\phi)=\mathcal{E}_{s}^{(0)}+\mathcal{E}_{s}^{(1)}=A(s) e^{-2 s \phi}+B(s) e^{-2(2-s) \phi} .
$$

Below we will compute the coefficients $A(s)$ and $B(s)$. The first constant term $\mathcal{E}_{s}^{(0)}$ corresponds to the leading order term in an expansion at the cusp $e^{\phi} \rightarrow 0$, which physically corresponds to the regime of weak coupling.

Our strategy for performing the Fourier expansion is as follows: we first consider the term $\omega_{3}=0$, which by virtue of the constraint (3.5) also requires $\omega_{2}=0$. The remaining sum over $\omega_{1} \neq 0$ yields the first constant term $\mathcal{E}_{s}^{(0)}$. We then consider the case $\omega_{3} \neq 0$ and solve the constraint (3.5) explicitly using the Euclidean algorithm, which reduces the remaining sum to one over three integers. On these integers we will perform Poisson resummations to uncover the second constant term, as well the Abelian and non-Abelian Fourier coefficients.

Accordingly, we start by extracting the $\omega_{3}=0$ (implying $\omega_{2}=0$ ) part of the sum in the Eisenstein series, $\mathcal{E}_{s}^{(0)}$, leaving a remainder $\mathcal{A}^{(s)}$

$$
\mathcal{E}_{s}(\phi, \lambda, \gamma)=\mathcal{E}_{s}^{(0)}+\mathcal{A}^{(s)}
$$

The first term $\mathcal{E}_{s}^{(0)}$ is the leading order contribution in the limit $e^{\phi} \rightarrow 0$ and corresponds to a sum over $\omega_{1}=m_{1}+i m_{2}$

$$
\mathcal{E}_{s}^{(0)}=e^{-2 s \phi} \sum_{\left(m_{1}, m_{2}\right) \in \mathbb{Z}^{2}}^{\prime} \frac{1}{\left(m_{1}^{2}+m_{2}^{2}\right)^{s}}=4 \zeta_{\mathbb{Q}(i)}(s) e^{-2 s \phi},
$$

where $\zeta_{\mathbb{Q}(i)}(s)$ is the Dedekind zeta function over the Gaussian integers

$$
\zeta_{\mathbb{Q}(i)}(s)=\frac{1}{4} \sum_{\omega \in \mathbb{Z}[i]}^{\prime}|\omega|^{-2 s}=\frac{1}{4} \sum_{(m, n) \in \mathbb{Z}^{2}}^{\prime} \frac{1}{\left(m^{2}+n^{2}\right)^{s}} .
$$

The factor of 4 is related to the units of the Gaussian integers (see Appendix A).

The Dedekind zeta function $\zeta_{\mathbb{Q}(i)}(s)$ satisfies a functional equation, which is most conveniently written in terms of the "completed Dedekind zeta function"

$$
\zeta_{\mathbb{Q}(i) *}(s):=\pi^{-s} \Gamma(s) \zeta_{\mathbb{Q}(i)}(s),
$$

in terms of which one has

$$
\zeta_{\mathbb{Q}(i) *}(1-s)=\zeta_{\mathbb{Q}(i) *}(s) .
$$


It is known that the Dedekind function over a quadratic number field can be written as a Dirichlet L-function times the standard Riemann zeta function. In our case this reads (see, e.g., [74] for a proof)

$$
\zeta_{\mathbb{Q}(i)}(s)=\beta(s) \zeta(s),
$$

where the standard Riemann zeta function is defined as

$$
\zeta(s):=\sum_{n=1}^{\infty} n^{-s}=\prod_{p \text { prime }} \frac{1}{1-p^{-s}} \quad \text { for } \Re(s)>1
$$

and $\beta(s)$ is the Dirichlet beta function, ${ }^{20}$

$$
\beta(s):=\sum_{n=0}^{\infty}(-1)^{n}(2 n+1)^{-s} \text { for } \Re(s)>0 .
$$

We also note that $\beta(s)$ has an Euler product representation of the form

$$
\beta(s)=\prod_{p: p=1 \bmod 4} \frac{1}{1-p^{-s}} \prod_{p: p=3 \bmod 4} \frac{1}{1+p^{-s}},
$$

which together with the Euler product form of the Riemann zeta function $\zeta(s)$ above will be useful later. The functional relation for $\beta(s)$ is again best stated using its completion

$$
\beta_{*}(s):=\left(\frac{\pi}{4}\right)^{-\frac{s+1}{2}} \Gamma\left(\frac{s+1}{2}\right) \beta(s),
$$

for which the functional relation takes the simple form

$$
\beta_{*}(s)=\beta_{*}(1-s) .
$$

In conclusion, we have found that the first coefficient $A(s)$ in (4.12) is given by the Dedekind function $\zeta_{\mathbb{Q}(i)}(s)$ and that it is related to the term $\omega_{3}=0$ in the sum over the Gaussian integers. We will now proceed to evaluate the terms with $\omega_{3} \neq 0$, contained in $\mathcal{A}^{(s)}$ of (4.13). We emphasize that the term with $\omega_{3}=0$ and $\omega_{2} \neq 0$ vanishes identically because of the quadratic constraint (3.5). Thus, $\mathcal{A}^{(s)}$ only contains terms for which $\omega_{3} \neq 0$.

\footnotetext{
${ }^{20}$ The Dirichlet beta function is also known as $L\left(\chi_{-4}, s\right)$, i.e., it is the $L$-function associated with the alternating character modulo 4 .
} 


\subsection{Solution of constraint and Poisson resummation}

To solve the constraint (3.5) we shall make use of the Euclidean algorithm, which implies that for integers $p_{1}$ and $p_{2}$ the equation

$$
q_{1} p_{2}-q_{2} p_{1}=d
$$

has integer solutions for $q_{1}$ and $q_{2}$ if and only if $d$ divides $\operatorname{gcd}\left(p_{1}, p_{2}\right)$. The most general solution is the sum of a particular solution $\left(q_{1}, q_{2}\right)$ plus an integer times $\left(p_{1}, p_{2}\right)$. More precisely, in the case of our constraint (3.5) we find that for $\omega_{3}=p_{1}+i p_{2} \neq 0$ there are solutions in $\mathbb{Z}[i]^{3}$ if and only if

$$
\frac{\left|\omega_{2}\right|^{2}}{2 d} \in \mathbb{Z}, \quad \text { where } d=\operatorname{gcd}\left(p_{1}, p_{2}\right)
$$

and the most general solution for $\omega_{1}=m_{1}+i m_{2}$ is then

$$
\begin{aligned}
& m_{1}=-\frac{\left|\omega_{2}\right|^{2}}{2 d} q_{1}+m \frac{p_{1}}{d} \\
& m_{2}=-\frac{\left|\omega_{2}\right|^{2}}{2 d} q_{2}+m \frac{p_{2}}{d}
\end{aligned}
$$

Here, $q_{1}$ and $q_{2}$ is any particular solution of $q_{1} p_{2}-q_{2} p_{1}=d$ and $m \in \mathbb{Z}$ is an unconstrained integer. Therefore, we can rewrite the constrained sum as

$$
\sum_{\omega_{3} \neq 0} \sum_{\substack{\left.\omega_{2} \in \mathbb{Z}[i] \\ 2 d|| \omega_{2}\right|^{2}}} \sum_{m \in \mathbb{Z}}(\cdots)
$$

where in the summand, $\omega_{1}=m_{1}+i m_{2}$ has to be replaced by the expression from (4.26).

Let us implement this procedure on our Eisenstein series. After solving the constraint, the first term in the bracket of (3.2) becomes

$$
\begin{aligned}
\left|\omega_{1}+\omega_{2} \lambda+\omega_{3} \gamma\right|^{2}= & \frac{\left|\omega_{3}\right|^{2}}{d^{2}}\left[\left(m-\frac{\left|\omega_{2}\right|^{2}}{2\left|\omega_{3}\right|^{2}}\left(q_{1} p_{1}+q_{2} p_{2}\right)+\tilde{\ell}_{1} \chi+\tilde{\ell}_{2} \tilde{\chi}+2 d \psi\right)^{2}\right. \\
& \left.+\frac{1}{16 d^{2}}\left(\left(\tilde{\ell}_{1}+2 d \tilde{\chi}\right)^{2}+\left(\tilde{\ell}_{2}-2 d \chi\right)^{2}\right)^{2}\right]
\end{aligned}
$$


where we defined

$$
\begin{aligned}
& \tilde{\ell}_{1}:=\frac{d}{\left|\omega_{3}\right|^{2}}\left[\left(p_{1}-p_{2}\right) n_{1}+\left(p_{1}+p_{2}\right) n_{2}\right] \\
& \tilde{\ell}_{2}:=\frac{d}{\left|\omega_{3}\right|^{2}}\left[\left(p_{1}+p_{2}\right) n_{1}-\left(p_{1}-p_{2}\right) n_{2}\right] .
\end{aligned}
$$

Extracting an overall factor of $\left|\omega_{3}\right|^{2} / d^{2}$, the total summand may be written as

$$
\begin{aligned}
\frac{d^{2}}{\left|\omega_{3}\right|^{2}} \omega^{\dagger} \cdot \mathcal{K} \cdot \omega= & {\left[m-\frac{\left|\omega_{2}\right|^{2}}{2\left|\omega_{3}\right|^{2}}\left(q_{1} p_{1}+q_{2} p_{2}\right)+\tilde{\ell}_{1} \chi+\tilde{\ell}_{2} \tilde{\chi}+2 d \psi\right]^{2} } \\
& +\frac{e^{-4 \phi}}{d^{2}}\left[d^{2}+\frac{e^{2 \phi}}{4}\left(\left(\tilde{\ell}_{1}+2 d \tilde{\chi}\right)^{2}+\left(\tilde{\ell}_{2}-2 d \chi\right)^{2}\right)\right]^{2}
\end{aligned}
$$

Using an integral representation for the summand in the remainder $\mathcal{A}^{(s)}$ defined in (4.13),

$$
\left[\vec{\omega}^{\dagger} \cdot \mathcal{K} \cdot \vec{\omega}\right]^{-s}=\frac{\pi^{s}}{\Gamma(s)} \int \frac{d t}{t^{s+1}} e^{-\frac{\pi}{t} \vec{\omega}^{\dagger} \cdot \mathcal{K} \cdot \vec{\omega}}
$$

and performing a Poisson resummation on $m$ using the standard formula

$$
\sum_{m \in \mathbb{Z}} e^{-\pi x(m+a)^{2}+2 \pi i m b}=\frac{1}{\sqrt{x}} \sum_{\tilde{m} \in \mathbb{Z}} e^{-\frac{\pi}{x}(\tilde{m}+b)^{2}-2 \pi i(\tilde{m}+b) a},
$$

we obtain

$$
\begin{aligned}
\mathcal{A}^{(s)}= & \frac{\pi^{s}}{\Gamma(s)} e^{-2 s \phi} \sum_{\tilde{m} \in \mathbb{Z}\left(p_{1}, p_{2}\right) \in \mathbb{Z}^{2}} \sum_{\substack{\left(n_{1}, n_{2}\right) \in \mathbb{Z}^{2} \\
2 d \mid n_{1}^{2}+n_{2}^{2}}}^{\prime} \\
& \times \frac{d}{\left|\omega_{3}\right|} e^{-2 \pi i \tilde{m}\left(-\frac{\left|\omega_{2}\right|^{2}}{2\left|\omega_{3}\right|^{2}}\left(q_{1} p_{1}+q_{2} p_{2}\right)+\tilde{\ell}_{1} \chi+\tilde{\ell}_{2} \tilde{\chi}+2 d \psi\right)} \\
& \times \int_{0}^{\infty} \frac{d t}{t^{s+1 / 2}} e^{-\pi t \frac{d^{2}}{\left|\omega_{3}\right|^{2}} \tilde{m}^{2}-\frac{\pi}{t} \frac{\left|\omega_{3}\right|^{2}}{d^{4}} e^{-4 \phi}\left[d^{2}+\frac{e^{2 \phi}}{4}\left(\left(\tilde{\ell}_{1}+2 d \tilde{\chi}\right)^{2}+\left(\tilde{\ell}_{2}-2 d \chi\right)^{2}\right)\right]^{2}},
\end{aligned}
$$

where we have indicated explicitly the constraint from (4.25) that $2 d$ must divide $\left|\omega_{2}\right|^{2}$.

As we will see in Section 5, the Abelian terms in the Fourier expansion correspond physically to instantons with zero NS5-brane charge, independent of the NS-NS scalar $\psi$. We therefore split off the Abelian contribution 
with $\tilde{m}=0$ :

$$
\mathcal{A}^{(s)}=\mathcal{D}^{(s)}+\mathcal{E}_{s}^{(\mathrm{NA})},
$$

where $\mathcal{E}_{s}^{(\mathrm{NA})}$ denotes the non-Abelian term with $\tilde{m} \neq 0$, to be considered later. From $\mathcal{D}^{(s)}$ we will be able to extract the second constant term $\mathcal{E}_{s}^{(1)}$ as well as the Abelian Fourier coefficients $\mathfrak{C}_{\ell_{1}, \ell_{2}}^{(\mathrm{A})}(s)$. Explicitly, we have

$$
\begin{aligned}
\mathcal{D}^{(s)}= & \frac{\pi^{s}}{\Gamma(s)} e^{-2 s \phi} \sum_{\substack {\left(p_{1}, p_{2}\right) \in \mathbb{Z}^{2} \\
\begin{subarray}{c}{\left(n_{1}, n_{2}\right) \in \mathbb{Z}^{2} \\
2 d \mid n_{1}^{2}+n_{2}^{2}{ ( p _ { 1 } , p _ { 2 } ) \in \mathbb { Z } ^ { 2 } \\
\begin{subarray} { c } { ( n _ { 1 } , n _ { 2 } ) \in \mathbb { Z } ^ { 2 } \\
2 d | n _ { 1 } ^ { 2 } + n _ { 2 } ^ { 2 } } }\end{subarray}} \frac{d}{\left|\omega_{3}\right|} \\
& \times \int_{0}^{\infty} \frac{d t}{t^{s+1 / 2}} e^{-\frac{\pi}{t} \frac{e^{-4 \phi}\left|\omega_{3}\right|^{2}}{d^{4}}\left[d^{2}+\frac{e^{2 \phi}}{4}\left(\left(\tilde{\ell}_{1}+2 d \tilde{\chi}\right)^{2}+\left(\tilde{\ell}_{2}-2 d \chi\right)^{2}\right)\right]^{2}} .
\end{aligned}
$$

To get rid of the square in the exponent, we shall perform the integration over $t$ and then choose a new integral representation of the summand. The current form of the exponent will be convenient for the evaluation of the non-Abelian terms in Section 4.6, but for our present purposes we shall rewrite it in the following way:

$$
\begin{aligned}
& \frac{e^{-4 \phi}\left|\omega_{3}\right|^{2}}{d^{4}}\left[d^{2}+\frac{e^{2 \phi}}{4}\left(\left(\tilde{\ell}_{1}+2 d \tilde{\chi}\right)^{2}+\left(\tilde{\ell}_{2}-2 d \chi\right)^{2}\right)\right]^{2} \\
& =\frac{1}{4\left|\omega_{3}\right|^{2}}\left[|\mathcal{Y}|^{2}+2 e^{-2 \phi}\left|\omega_{3}\right|^{2}\right]^{2}
\end{aligned}
$$

where we defined the new variable $\mathcal{Y}=\mathcal{Y}_{1}+i \mathcal{Y}_{2}$, with

$$
\begin{aligned}
& \mathcal{Y}_{1}:=n_{1}+\left(p_{1}-p_{2}\right) \tilde{\chi}-\left(p_{1}+p_{2}\right) \chi \\
& \mathcal{Y}_{2}:=n_{2}+\left(p_{1}+p_{2}\right) \tilde{\chi}+\left(p_{1}-p_{2}\right) \chi
\end{aligned}
$$

Evaluating the integral over $t$ then yields

$$
\begin{aligned}
\mathcal{D}^{(s)}= & \frac{2^{2 s-1} \sqrt{\pi} \Gamma(s-1 / 2)}{\Gamma(s)} e^{-2 s \phi} \sum_{\left(p_{1}, p_{2}\right) \in \mathbb{Z}^{2}}^{\prime} \sum_{\substack{\left(n_{1}, n_{2}\right) \in \mathbb{Z}^{2} \\
2 d \mid n_{1}^{2}+n_{2}}} \\
& \times \frac{d}{\left|\omega_{3}\right|^{2-2 s}}\left\{\left[|\mathcal{Y}|^{2}+2 e^{-2 \phi}\left|\omega_{3}\right|^{2}\right]\right\}^{1-2 s} .
\end{aligned}
$$


After replacing the term within brackets by its integral representation, we obtain

$$
\begin{aligned}
\mathcal{D}^{(s)}= & \frac{(2 \pi)^{2 s-1} \sqrt{\pi} \Gamma(s-1 / 2)}{\Gamma(s) \Gamma(2 s-1)} e^{-2 s \phi} \sum_{\left(p_{1}, p_{2}\right) \in \mathbb{Z}^{2}}^{\prime} \sum_{\substack{\left(n_{1}, n_{2}\right) \in \mathbb{Z}^{2} \\
2 d \mid n_{1}^{2}+n_{2}}} \frac{d}{\left|\omega_{3}\right|^{2-2 s}} \\
& \times \int_{0}^{\infty} \frac{d t}{t^{2 s}} e^{-\frac{\pi}{t}\left[|\mathcal{Y}|^{2}+2 e^{-2 \phi}\left|\omega_{3}\right|^{2}\right]} .
\end{aligned}
$$

Since all values of $n_{1}$ and $n_{2}$ are almost degenerate we shall perform a further Poisson resummation on these variables. Here we must take into account the remaining constraint that $2 d$ divides $n_{1}^{2}+n_{2}^{2}$. The set of solutions to this constraint can be written as

$$
n_{1}=n_{1}^{0}+\delta n_{1}, \quad n_{2}=n_{2}^{0}+\delta n_{2},
$$

where $\delta n_{1}+i \delta n_{2}$ runs over the lattice $L$

$$
L=\left\{d\left[\left(k_{1}+k_{2}\right)+i\left(k_{1}-k_{2}\right)\right]:\left(k_{1}, k_{2}\right) \in \mathbb{Z}^{2}\right\}
$$

and $\left(n_{1}^{0}, n_{2}^{0}\right)$ runs over all solutions of the quadratic equation $n_{1}^{2}+n_{2}^{2}=$ 0 mod $2 d$ in a fundamental domain of $\mathbb{Z}[i] / L$, which we take to be $0 \leq n_{1}^{0}<d$ and $0 \leq n_{2}^{0}<2 d$, with area $2 d^{2}$. We denote the set of such solutions as

$$
\mathcal{F}(d):=\left\{n_{1}^{0}+i n_{2}^{0}: n_{1}^{2}+n_{2}^{2}=0 \bmod 2 d, 0 \leq n_{1}^{0}<d, 0 \leq n_{2}^{0}<2 d\right\},
$$

and its cardinality by

$$
N(d):=\sharp \mathcal{F}(d)
$$

As we shall discuss below and in Appendix C, the series $N(d)$ is multiplicative though not completely multiplicative (see [75] for the first few values).

After inserting (4.40) into (4.39) and performing a Poisson resummation on $\delta n_{1}$ and $\delta n_{2}$, we obtain

$$
\begin{aligned}
\mathcal{D}^{(s)}= & \frac{(2 \pi)^{2 s-1} \sqrt{\pi} \Gamma(s-1 / 2)}{2 \Gamma(s) \Gamma(2 s-1)} e^{-2 s \phi} \sum_{\left(p_{1}, p_{2}\right) \in \mathbb{Z}^{2}}^{\prime} \sum_{\tilde{\omega}_{2} \in L^{*}} \sum_{f \in \mathcal{F}(d)} \frac{1}{d\left|\omega_{3}\right|^{2-2 s}} \\
& \times e^{2 \pi i \Re\left(\tilde{\omega}_{2} f\right)} \int_{0}^{\infty} \frac{d t}{t^{2 s-1}} e^{-\pi t\left(\tilde{n}_{1}^{2}+\tilde{n}_{2}^{2}\right)-\frac{2 \pi}{t} e^{-2 \phi}\left|\omega_{3}\right|^{2}+2 \pi i\left(\ell_{1} \chi+\ell_{2} \tilde{\chi}\right)}
\end{aligned}
$$


where $L^{*}$ is the lattice dual to $L$,

$$
L^{*}=\left\{\tilde{\omega}_{2}=\tilde{n}_{1}+i \tilde{n}_{2}=\frac{1}{2 d}\left[\left(\tilde{k}_{1}+\tilde{k}_{2}\right)+i\left(\tilde{k}_{1}-\tilde{k}_{2}\right)\right]:\left(\tilde{k}_{1}, \tilde{k}_{2}\right) \in \mathbb{Z}^{2}\right\},
$$

and we defined the new charges

$$
\begin{aligned}
\ell_{1} & :=\tilde{n}_{1}\left(p_{1}+p_{2}\right)-\tilde{n}_{2}\left(p_{1}-p_{2}\right), \\
\ell_{2} & :=\tilde{n}_{1}\left(p_{2}-p_{1}\right)-\tilde{n}_{2}\left(p_{1}+p_{2}\right) .
\end{aligned}
$$

\subsection{Second constant term}

We may now extract the second constant term from the $\ell_{1}=\ell_{2}=0$ part of the sum, and accordingly we split $\mathcal{D}^{(s)}$ as

$$
\mathcal{D}^{(s)}=\mathcal{E}_{s}^{(1)}+\mathcal{E}_{s}^{(\mathrm{A})}
$$

where $\mathcal{E}_{s}^{(\mathrm{A})}$ is the Abelian term in the Fourier expansion to be considered in the next subsection. The $\ell_{1}=\ell_{2}=0$ part arises from the $\tilde{\omega}_{2}=0$ term, which reads

$$
\begin{aligned}
\mathcal{E}_{s}^{(1)}= & \frac{(2 \pi)^{2 s-1} \Gamma(s-1 / 2)}{2 \sqrt{\pi} \Gamma(s) \Gamma(2 s-1)} e^{-2 s \phi} \sum_{\left(p_{1}, p_{2}\right) \in \mathbb{Z}^{2}}^{\prime} \sum_{f \in \mathcal{F}(d)} \frac{1}{d\left|\omega_{3}\right|^{2-2 s}} \\
& \times \int_{0}^{\infty} \frac{d t}{t^{2 s-1}} e^{-\frac{2 \pi}{t} e^{-2 \phi}\left|\omega_{3}\right|^{2}} .
\end{aligned}
$$

The sum over $f \in \mathcal{F}(d)$ produces the multiplicative function $N(d)$ in (4.43). The integral can be explicitly evaluated with the result

$$
\mathcal{E}_{s}^{(1)}=\frac{\pi^{3 / 2} \Gamma(s-1 / 2) \Gamma(2 s-2)}{\Gamma(s) \Gamma(2 s-1)} e^{-2(2-s) \phi} \sum_{\left(p_{1}, p_{2}\right) \in \mathbb{Z}^{2}}^{\prime} N(d) \frac{1}{d\left|\omega_{3}\right|^{2 s-2}}
$$

The sum can now be expressed in terms of the Riemann zeta function and Dedekind zeta function (4.15) as follows. Extract the greatest common divisor of $p_{1}$ and $p_{2}$, defining $p_{1}=d p_{1}^{\prime}$ and $p_{2}=d p_{2}^{\prime}$, with $d=\operatorname{gcd}\left(p_{1}, p_{2}\right)$ and $\operatorname{gcd}\left(p_{1}^{\prime}, p_{2}^{\prime}\right)=1$. This yields a sum over $d$ and coprime $\left(p_{1}^{\prime}, p_{2}^{\prime}\right)$

$$
\sum_{\left(p_{1}, p_{2}\right) \in \mathbb{Z}^{2}}^{\prime} N(d) d^{-1}|p|^{2-2 s}=\left(\sum_{d>0} N(d) d^{1-2 s}\right)\left(\sum_{\left(p_{1}^{\prime}, p_{2}^{\prime}\right)=1} \frac{1}{\left(p_{1}^{\prime 2}+p_{2}^{\prime 2}\right)^{s-1}}\right) .
$$


The second sum may be rewritten as a ratio of Riemann and Dedekind zeta functions as follows (see Section 4.2):

$$
\sum_{\left(p_{1}^{\prime}, p_{2}^{\prime}\right)=1} \frac{1}{\left(p_{1}^{\prime 2}+p_{2}^{\prime 2}\right)^{s-1}}=\frac{4 \zeta_{\mathbb{Q}(i)}(s-1)}{\zeta(2 s-2)} .
$$

Let us now consider the first sum on the right-hand side of (4.50), which involves the combinatorial function $N(d)$ defined in (4.43) (see also [75]). Given $N(d)$ we may construct the Dirichlet series

$$
L(N, s):=\sum_{d=1}^{\infty} N(d) d^{-s}
$$

that converges for $\Re(s)>2$. Since $N(d)$ is multiplicative, we may evaluate $L(N, s)$ using Euler products. To this end we note that the multiplicative series exhibits the following properties (mentioned in [75], and derived in greater generality in Appendix C):

$$
N\left(2^{m}\right)=2^{m}, \quad N\left(p^{m}\right)=\left\{\begin{array}{cl}
(m(p-1)+p) p^{m-1}, & p=1 \bmod 4 \\
p^{2\lfloor m / 2\rfloor}, & p=3 \bmod 4 .
\end{array}\right.
$$

Therefore, the Dirichlet series (4.52) has an Euler product representation given by (see Appendix A for the derivation)

$$
L(N, s)=\frac{1}{1-2^{1-s}} \prod_{p: p=1 \bmod 4} \frac{1-p^{-s}}{\left(1-p^{1-s}\right)^{2}} \prod_{p: p=3 \bmod 4} \frac{1+p^{-s}}{\left(1-p^{1-s}\right)\left(1+p^{1-s}\right)},
$$

where the product runs over all primes $p>2$. Comparing with (4.19) and (4.21), we deduce that

$$
L(N, s)=\frac{\beta(s-1) \zeta(s-1)}{\beta(s)} .
$$

Putting everything together we then find the following expression for the constant term:

$$
\mathcal{E}_{s}^{(1)}=4 \frac{\pi^{3 / 2} \Gamma(s-1 / 2) \Gamma(2 s-2)}{\Gamma(s) \Gamma(2 s-1)} \frac{L(N, 2 s-1)}{\zeta(2 s-2)} \zeta_{\mathbb{Q}(i)}(s-1) e^{-2(2-s) \phi}
$$

Referring back to the completed Dedekind zeta function (4.16) and Dirichlet beta function (4.22), we define a completed "Picard Zeta function" by

$$
\mathfrak{Z}(s):=\zeta_{\mathbb{Q}(i) *}(s) \beta_{*}(2 s-1),
$$


in terms of which the two constant terms can be neatly summarized by

$$
\mathcal{E}_{s}^{(\text {const })}=\mathcal{E}_{s}^{(0)}+\mathcal{E}_{s}^{(1)}=4 \zeta_{\mathbb{Q}(i)}(s)\left\{e^{-2 s \phi}+\frac{\mathfrak{Z}(2-s)}{\mathfrak{Z}(s)} e^{-2(2-s) \phi}\right\}
$$

Equation (4.58) can be viewed as an extension of Langlands's constant term formula [65] for Eisenstein series associated to special linear groups to the case of the unitary group $S U(2,1)$. The completed Picard zeta function $\mathfrak{Z}(s)$ plays the same role as the completed Riemann zeta function $\xi(s)=$ $\pi^{-s / 2} \Gamma(s / 2) \zeta(s)$ in Langlands' formula.

\subsection{Abelian Fourier coefficients}

We now turn to the Abelian Fourier coefficients, corresponding to the terms $\left(\tilde{n}_{1}, \tilde{n}_{2}\right) \neq 0$ in (4.44). The integral over $t$ leads to a modified Bessel function,

$$
\begin{aligned}
\mathcal{E}_{s}^{(\mathrm{A})}= & \frac{2 \pi^{2 s-1 / 2} \Gamma(s-1 / 2)}{\Gamma(s) \Gamma(2 s-1)} e^{-2 \phi} \sum_{\left(p_{1}, p_{2}\right) \in \mathbb{Z}^{2}}^{\prime} \sum_{\left(\tilde{k}_{1}, \tilde{k}_{2}\right) \in \mathbb{Z}^{2}}^{\prime} \sum_{f \in \mathcal{F}(d)} \frac{1}{d^{2 s-1}}|u|^{2 s-2} \\
& \times e^{\frac{\pi i}{d} \Re[u f(1-i)]} K_{2 s-2}\left(2 \pi e^{-\phi}|\Lambda|\right) e^{2 \pi i\left(\ell_{1} \chi+\ell_{2} \tilde{\chi}\right)}
\end{aligned}
$$

where we have introduced the following additional notation:

$$
u=\tilde{k}_{1}+i \tilde{k}_{2}, \quad \Lambda=\ell_{2}-i \ell_{1}
$$

for

$$
\ell_{1}=\frac{1}{d}\left(\tilde{k}_{1} p_{2}+\tilde{k}_{2} p_{1}\right), \quad \ell_{2}=\frac{1}{d}\left(\tilde{k}_{2} p_{2}-\tilde{k}_{1} p_{1}\right)
$$

These charges are manifestly integral since $d$ divides $p_{1}$ and $p_{2}$. This last relation can also be written as

$$
\Lambda=\frac{u \omega_{3}}{d}=u \omega_{3}^{\prime}
$$

where $\omega_{3}^{\prime}=\omega_{3} / d$ is a primitive Gaussian number (i.e., a Gaussian number whose real and imaginary parts are coprime). To extract the Abelian Fourier coefficients $\mathfrak{C}_{\ell_{1}, \ell_{2}}^{(\mathrm{A})}(\phi)$ we therefore replace the sum over $\omega_{3}$ and $u$ by a sum over 
$d, \Lambda$ and $\omega_{3}^{\prime}$ where the primitive Gaussian integer $\omega_{3}^{\prime}$ has to be a Gaussian divisor of $\Lambda$, to wit

$$
\begin{aligned}
& \mathcal{E}_{s}^{(\mathrm{A})}=C_{s}^{(\mathrm{A})} e^{-2 \phi} \sum_{\Lambda \in \mathbb{Z}[i]}^{\prime}\left\{\sum_{\omega_{3}^{\prime} \mid \Lambda}\left|\frac{\Lambda}{\omega_{3}^{\prime}}\right|^{2 s-2}\left(\sum_{d>0} \frac{1}{d^{2 s-1}} \sum_{f \in \mathcal{F}(d)} e^{\frac{\pi i}{d} \Re\left[\frac{\Lambda}{\omega_{3}^{\prime}} f(1-i)\right]}\right)\right\} \\
& (4.63) \quad \times K_{2 s-2}\left(2 \pi e^{-\phi}|\Lambda|\right) e^{2 \pi i\left(\ell_{1} \chi+\ell_{2} \tilde{\chi}\right)},
\end{aligned}
$$

where the coefficient is given by

$$
C_{s}^{(\mathrm{A})}=\frac{2 \pi^{2 s-1 / 2} \Gamma(s-1 / 2)}{\Gamma(s) \Gamma(2 s-1)}=\frac{8 \zeta_{\mathbb{Q}(i)}(s) \beta(2 s-1)}{\mathfrak{Z}(s)} .
$$

To make contact with the general discussion of Section 4.1, we rewrite this result as a sum over the real variables $\ell_{1}$ and $\ell_{2}$ :

$$
\begin{aligned}
\mathcal{E}_{s}^{(\mathrm{A})}= & 2 \zeta_{\mathbb{Q}(i)}(s) \frac{e^{-2 \phi}}{\mathfrak{Z}(s)} \sum_{\left(\ell_{1}, \ell_{2}\right) \in \mathbb{Z}^{2}}^{\prime} \mu_{s}\left(\ell_{1}, \ell_{2}\right)\left[\ell_{1}^{2}+\ell_{2}^{2}\right]^{s-1} \\
& \times K_{2 s-2}\left(2 \pi e^{-\phi} \sqrt{\ell_{1}^{2}+\ell_{2}^{2}}\right) e^{2 \pi i\left(\ell_{1} \chi+\ell_{2} \tilde{\chi}\right)},
\end{aligned}
$$

where we defined the summation measure

$$
\mu_{s}\left(\ell_{1}, \ell_{2}\right):=4 \beta(2 s-1) \sum_{\omega_{3}^{\prime} \mid \Lambda}\left|\omega_{3}^{\prime}\right|^{2-2 s}\left(\sum_{d>0} d^{1-2 s} \sum_{f \in \mathcal{F}(d)} e^{\frac{\pi i}{d} \Re\left[\frac{\Lambda}{\omega_{3}^{\prime}} f(1-i)\right]}\right),
$$

containing the sum over primitive Gaussian divisors of $\Lambda=\ell_{2}-i \ell_{1}$. The sum over $d$ in the parenthesis may be carried out for fixed $\Lambda$ and $\omega_{3}^{\prime}$ to give the Gaussian divisor function (see Appendix $\mathrm{C}$ for the derivation)

$$
\sum_{d>0} d^{1-2 s} \sum_{f \in \mathcal{F}(d)} e^{\frac{\pi i}{d} \Re\left[\frac{\Lambda}{\omega_{3}^{\prime}} f(1-i)\right]}=\frac{1}{4 \beta(2 s-1)} \sum_{z \mid \frac{\Lambda}{\omega_{3}^{\prime}}}|z|^{4-4 s},
$$

whence the instanton measure (4.66) simplifies to

$$
\mu_{s}\left(\ell_{1}, \ell_{2}\right)=\sum_{\omega_{3}^{\prime} \mid \Lambda}\left|\omega_{3}^{\prime}\right|^{2-2 s} \sum_{z \mid \frac{\Lambda}{\omega_{3}^{\prime}}}|z|^{4-4 s} .
$$

Thus, the Abelian summation measure (4.68) involves both a sum over primitive divisors of $\Lambda$ and a sum over all divisors of $\Lambda / \omega_{3}^{\prime}$. By comparing (4.65) 
with (4.9) we may now extract the numerical Abelian Fourier coefficients:

$$
C_{\ell_{1}, \ell_{2}}^{(\mathrm{A})}(s)=\frac{2 \zeta_{\mathbb{Q}(i)}(s)}{\mathfrak{Z}(s)} \mu_{s}\left(\ell_{1}, \ell_{2}\right)\left[\ell_{1}^{2}+\ell_{2}^{2}\right]^{s-1} .
$$

We note that the Abelian instanton measure (4.68) is multiplicative in a restricted sense: The relation

$$
\mu_{s}\left(\Lambda_{1}\right) \mu_{s}\left(\Lambda_{2}\right)=\mu_{s}\left(\Lambda_{1} \Lambda_{2}\right)
$$

holds if (and only if) the Gaussian integers $\Lambda_{1}$ and $\Lambda_{2}$ admit no common prime factor up to complex conjugation (this caveat is relevant for split primes, see Appendix A).

\subsection{Non-Abelian Fourier coefficients}

Finally, we consider the non-Abelian term $\mathcal{E}_{s}^{(\mathrm{NA})}$ in (4.34). This term reads

$$
\begin{aligned}
& \mathcal{E}_{s}^{(\mathrm{NA})}=\frac{\pi^{s}}{\Gamma(s)} e^{-2 s \phi} \sum_{\tilde{m} \in \mathbb{Z}}^{\prime} \sum_{\left(p_{1}, p_{2}\right) \in \mathbb{Z}^{2}}^{\prime} \sum_{\substack{\left.n_{1}, n_{2}\right) \in \mathbb{Z}^{2} \\
2 d \mid n_{1}^{2}+n_{2}^{2}}} \\
& \times \frac{d}{\left|\omega_{3}\right|} e^{-2 \pi i \tilde{m}\left(-\frac{\left|\omega_{2}\right|^{2}}{2\left|\omega_{3}\right|^{2}}\left(q_{1} p_{1}+q_{2} p_{2}\right)+\tilde{\ell}_{1} \chi+\tilde{\ell}_{2} \tilde{\chi}+2 d \psi\right)}
\end{aligned}
$$

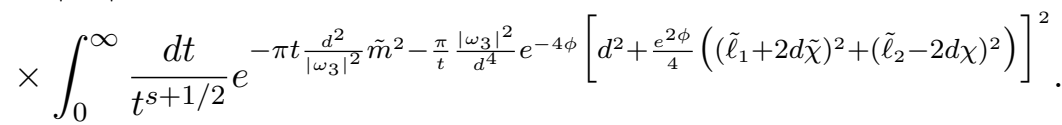

The integral is of Bessel type and yields

$$
\begin{aligned}
\mathcal{E}_{s}^{(\mathrm{NA})}= & \frac{2 \pi^{s}}{\Gamma(s)} e^{-2 s \phi} \sum_{\tilde{m} \in \mathbb{Z}}^{\prime} \sum_{\left(p_{1}, p_{2}\right) \in \mathbb{Z}^{2}}^{\prime} \sum_{\substack{\left(n_{1}, n_{2}\right) \in \mathbb{Z}^{2} \\
2 d \mid n_{1}^{2}+n_{2}^{2}}}\left[\frac{d}{\left|\omega_{3}\right|}\right]^{s+1 / 2}\left[\frac{|\tilde{m}|^{2}}{\Re\left(S_{\ell_{1}, \ell_{2}, k}\right)}\right]^{s-1 / 2} \\
& \times K_{s-1 / 2}\left(2 \pi \Re\left(S_{\ell_{1}, \ell_{2}, k}\right)\right) e^{-2 \pi i \Im\left(S_{\ell_{1}, \ell_{2}, k}\right)} e^{-\frac{\pi i}{2 k d}\left(\ell_{1}^{2}+\ell_{2}^{2}\right)\left(q_{1} p_{1}+q_{2} p_{2}\right)},
\end{aligned}
$$

where the real and imaginary parts of $S_{\ell_{1}, \ell_{2}, k}$ are given by

$$
\begin{aligned}
& \Re\left(S_{\ell_{1}, \ell_{2}, k}\right)=|k| e^{-2 \phi}+\frac{1}{4|k|}\left[\left(\ell_{1}+2 k \tilde{\chi}\right)^{2}+\left(\ell_{2}-2 k \chi\right)^{2}\right], \\
& \Im\left(S_{\ell_{1}, \ell_{2}, k}\right)=\ell_{1} \chi+\ell_{2} \tilde{\chi}+2 k \psi,
\end{aligned}
$$


and we also defined ${ }^{21}$

$$
\begin{aligned}
k & :=\tilde{m} d \\
\ell_{1} & :=\frac{k}{\left|\omega_{3}\right|^{2}}\left[\left(p_{1}-p_{2}\right) n_{1}+\left(p_{1}+p_{2}\right) n_{2}\right] \\
\ell_{2} & :=\frac{k}{\left|\omega_{3}\right|^{2}}\left[\left(p_{1}+p_{2}\right) n_{1}-\left(p_{1}-p_{2}\right) n_{2}\right] .
\end{aligned}
$$

In Gaussian notation, $\Lambda=\ell_{2}-i \ell_{1}$, the last two relations amount to

$$
\bar{\Lambda}=\frac{(1+i) k \omega_{2}}{\omega_{3}} .
$$

Comparing the expression (4.72) with the general form of the non-Abelian term (4.10), we see that the former involves a sum of nearly Gaussian wavefunctions peaked around $\left(\ell_{2},-\ell_{1}\right) /(2 k)$ in the $(\chi, \tilde{\chi})$ plane, while the latter is written in terms of a basis of Landau-type wave functions which are eigenmodes of $\partial_{\chi}$ and $\partial_{\psi+\chi}$, with quantized charges $4 k n$ and $k$. To extract the non-Abelian Fourier coefficients $C_{r, k, \ell}^{(\mathrm{NA})}(s)$ we must therefore transform (4.72) into the correct basis. This can be achieved via Fourier transform along the variable $\chi$ (or $\tilde{\chi}$ in the other polarization).

To perform the Fourier transform we go back to the integral representation in (4.71). The integrand is quartic in $\chi$ and therefore inconvenient for Fourier transform. To remedy this we make the following change of integration variables:

$$
t=\frac{t^{\prime}\left|\omega_{3}\right|^{2} A}{k^{2}}
$$

where

$$
A(y, \chi, \tilde{\chi})=k\left[y+\left(\tilde{\chi}+\frac{\ell_{1}}{2 k}\right)^{2}+\left(\chi-\frac{\ell_{2}}{2 k}\right)^{2}\right]
$$

and we recall that $y=e^{-2 \phi}$. Implementing this in (4.71), and denoting $\tilde{\ell}_{i}=$

${ }^{21}$ The non-Abelian charges $\ell_{i}$ defined in (??) should not be confused with the Abelian charges $\ell_{i}$ in (4.46). 
$\ell_{i} / \tilde{m}$, we obtain

$$
\begin{aligned}
\mathcal{E}_{s}^{(\mathrm{NA})}= & \frac{\pi^{s}}{\Gamma(s)} y^{s} \sum_{\tilde{m} \in \mathbb{Z}}^{\prime} \sum_{\left(p_{1}, p_{2}\right) \in \mathbb{Z}^{2}}^{\prime} \sum_{\substack{\left(n_{1}, n_{2}\right) \in \mathbb{Z}^{2} \\
2 d \mid n_{1}^{2}+n_{2}^{2}}} \frac{d|k|^{2 s-1}}{\left|\omega_{3}\right|^{2 s}} \\
& \times e^{-2 \pi i \tilde{m}\left(-\frac{\left|\omega_{2}\right|^{2}}{2\left|\omega_{3}\right|^{2}}\left(q_{1} p_{1}+q_{2} p_{2}\right)+\tilde{\ell}_{1} \chi+\tilde{\ell}_{2} \tilde{\chi}+2 d \psi\right)} \\
& \times \int_{0}^{\infty} \frac{d t^{\prime}}{t^{\prime s+1 / 2}} A^{1 / 2-s} e^{-\pi\left(t^{\prime}+\frac{1}{t^{\prime}}\right) A}
\end{aligned}
$$

where the exponent is now quadratic in both $\chi$ and $\tilde{\chi}$. Using an integral representation for the factor $A^{1 / 2-s}$, and dropping the prime on $t^{\prime}$, we may rewrite this expression as follows:

$$
\begin{aligned}
& \mathcal{E}_{s}^{(\mathrm{NA})}=\frac{\pi^{2 s-1 / 2}}{\Gamma(s) \Gamma(s-1 / 2)} y^{s} \sum_{\tilde{m} \in \mathbb{Z}}^{\prime} \sum_{\left(p_{1}, p_{2}\right) \in \mathbb{Z}^{2}}^{\prime} \sum_{\substack{\left(n_{1}, n_{2}\right) \in \mathbb{Z}^{2} \\
2 d \mid n_{1}^{2}+n_{2}^{2}}} \frac{d|k|^{2 s-1}}{\left|\omega_{3}\right|^{2 s}} e^{\frac{\pi i \tilde{m}\left|\omega_{2}\right|^{2}}{2\left|\omega_{3}\right|^{2}}\left(q_{1} p_{1}+q_{2} p_{2}\right)} \\
& \times e^{-4 \pi i k \psi-2 \pi i \ell_{2} \tilde{\chi}} \int_{0}^{\infty} \frac{d t d u}{t^{s+1 / 2} u^{3 / 2-s}} \\
& \times e^{-\pi k\left(u+t+\frac{1}{t}\right)\left[y+\left(\tilde{\chi}+\frac{\ell_{1}}{2 k}\right)^{2}\right]} f(y, \chi, \tilde{\chi} ; t, u),
\end{aligned}
$$

where all the $\chi$-dependence is contained in the function

$$
f(y, \chi ; t, u)=e^{-\pi k\left(u+t+\frac{1}{t}\right)\left(\chi-\frac{\ell_{2}}{2 k}\right)^{2}-2 \pi i \ell_{1} \chi} .
$$

The Fourier transform over $\chi$ is now implemented by substituting

$$
f(y, \chi ; t, u)=e^{-4 \pi i k \chi \tilde{\chi}} \int d n \hat{f}(y, n, \tilde{\chi} ; t, u) e^{8 \pi i k n \chi},
$$

with

$$
\begin{aligned}
\hat{f}(y, n, \tilde{\chi} ; t, u) & =4|k| \int d \xi e^{-8 \pi i k n \xi+4 \pi i k \xi \tilde{\chi}} f(y, \xi, \tilde{\chi} ; t, u) \\
& =4|k| e^{-\frac{\pi}{k} \frac{\left(\ell_{1}-2 k \tilde{\chi}+8 \pi k n\right)^{2}}{u+t+1 / t}}
\end{aligned}
$$


After Fourier transform, the non-Abelian term thus becomes

$$
\begin{aligned}
\mathcal{E}_{s}^{(\mathrm{NA})}= & \frac{4 \pi^{2 s-1 / 2}}{\Gamma(s) \Gamma(s-1 / 2)} y^{s} \sum_{\tilde{m} \in \mathbb{Z}}^{\prime} \sum_{\left(p_{1}, p_{2}\right) \in \mathbb{Z}^{2}}^{\prime} \sum_{\substack{\left(n_{1}, n_{2}\right) \in \mathbb{Z}^{2} \\
2 d \mid n_{1}^{2}+n_{2}^{2}}} \frac{d|k|^{2 s}}{\left|\omega_{3}\right|^{2 s}} e^{\frac{\pi i \tilde{m}\left|\omega_{2}\right|^{2}}{2\left|\omega_{3}\right|^{2}}\left(q_{1} p_{1}+q_{2} p_{2}\right)} \\
& \times \int \frac{d t d u}{t^{s+1 / 2} u^{3 / 2-s}} e^{-\pi k\left(u+t+\frac{1}{t}\right)\left[y+\left(\tilde{\chi}+\frac{\ell_{1}}{2 k}\right)^{2}\right]} \\
& \times \int d n e^{-\frac{\pi i}{k} \ell_{2}\left(\ell_{1}-4 k n\right)} e^{-\frac{\pi}{k} \frac{\left(\ell_{1}-2 k \tilde{\chi}+8 \pi k n\right)^{2}}{u+t+1 / t}} e^{8 \pi i k n \chi-4 \pi i k(\psi+\chi \tilde{\chi})} .
\end{aligned}
$$

Let us now comment on the structure of Equation (4.83). After Fourier transforming we see that the non-Abelian term indeed corresponds to an expansion in terms of the invariant wavefunctions on the twisted torus as in Equation (4.10). However, we have not been able to further manipulate Equation (4.83) into the form displayed in (4.10) and therefore we cannot extract the numerical Fourier coefficients $C_{k, \ell}^{(\mathrm{NA})}(s)$ in as compact a form as the Abelian coefficients (4.69). Nevertheless, as a consistency check we shall show that the leading order exponential behavior of (4.83) near the cusp $y \rightarrow \infty$ coincides with that of Equation (4.10). To this end we may take the saddle point approximation for the integrals over $t$ and $u$ in (4.83) for which the saddle points are located at $t=1$ and $u=0$. We thus find that the leading exponential dependence of (4.83) at the saddle point is given by $e^{-S}$ with

$$
\Re(S)=2 \pi|k|\left[y+\left(\tilde{\chi}+\frac{\ell_{1}}{2|k|}\right)^{2}\right]+\frac{\pi}{2|k|}\left(\ell_{1}-2|k| \tilde{\chi}+4|k| n\right)^{2} .
$$

Rearranging terms, this can be written as

$$
\Re(S)=2 \pi|k| y+4 \pi|k|(\tilde{\chi}-n)^{2}+4 \pi|k|\left(n+\frac{\ell_{1}}{2|k|}\right)^{2} .
$$

Using the asymptotic behavior of the Whittaker function $W_{k, m}(x) \sim e^{-x / 2}$ one may indeed verify that the first two terms in (4.85) exactly coincide with the leading behavior of the general expression (4.10) in the limit $y \rightarrow \infty$. We further expect that the summation over $\ell_{1}$ and $\ell_{2}$ (or, more precisely, over $\omega_{2}$ and $\left.\omega_{3}\right)$ will restrict the integral over $n$ such that it localizes on the points in $\mathbb{Z}+\ell /(4|k|)$ as is expected from the general expression (4.10). We stress that the result (4.85) is valid in the polarization (4.4) we have chosen. There is an analogous result for the other polarization.

Besides the representation of the non-Abelian coefficients in the form (4.83) one could also try to extract the coefficients by other means. One 
possibility would be to manipulate the expression (4.72) by expanding out the Bessel function and binomially expanding the resulting power series in $A(y, \chi, \tilde{\chi})$ to make contact with the power series expansions of the Hermite polynomials and Whittaker functions of (4.10). Alternatively, one could try to compute the coefficients by going to a suitably chosen point in moduli space (e.g., the cusp $y=\infty$ ) or by using $S U(2,1 ; \mathbb{Z}[i])$ symmetry or Hecke operators to relate the non-Abelian coefficients to the Abelian ones. We hope to present a complete investigation of the non-Abelian coefficients in a future publication.

\subsection{Functional relation}

Expression (4.58) for the constant terms of the Eisentein series $\mathcal{E}_{s}$ is suggestive of a functional relation most conveniently written in terms of the Poincaré series (3.11) and the Picard Zeta function (4.57),

$$
\mathfrak{Z}(s) \mathcal{P}_{s}=\mathfrak{Z}(2-s) \mathcal{P}_{2-s} .
$$

Indeed, it is easily checked that both the constant terms (4.58) and the Abelian Fourier coefficients (4.65), (4.68) satisfy this relation, taking into account the symmetry of the modified Bessel function $K_{2 s-2}(x)=K_{2-2 s}(x)$. Unfortunately, due to the unwieldy form of the non-Abelian terms we are unable to present a full proof of (4.86), which would constitute an analog of the familiar functional relation for Eisenstein series associated to special linear groups [65]. We note that a different functional relation for $\mathcal{P}_{s}$ has been proposed in [66] but this appears to contradict the constant term formula (4.58).

\section{Instanton corrections to the universal hypermultiplet}

In this section we propose that the Eisenstein series for the Picard modular group constructed in Section 3, and further analyzed in Section 4, controls the exact metric on the universal hypermultiplet moduli space $\mathcal{M}_{\mathrm{UH}}$, including the D2- and NS5-brane instanton corrections. We start by recalling some aspects of quantum corrections to hypermultiplet moduli spaces in type II Calabi-Yau compactifications, with particular emphasis on recent developments involving twistor techniques. 


\subsection{Twistor techniques for quaternionic-Kähler spaces}

Quantum corrections to the hypermultiplet moduli space are most conveniently described using twistor techniques $[22,24,28,29]$. Given a quaternionKähler space $\mathcal{M}$, one may construct its twistor space $\mathcal{Z}_{\mathcal{M}}$, a $\mathbb{C} P^{1}$ bundle over $\mathcal{M}$ which admits a canonical complex structure $\mathcal{J}$, a complex contact structure $\mathcal{C}$, a compatible real structure $\tau$ and a Kähler-Einstein metric $d s_{\mathcal{Z}_{\mathcal{M}}}^{2}$ with Kähler potential $K_{\mathcal{Z}_{\mathcal{M}}}$. The contact one-form $\mathcal{C}^{[i]}$ is proportional to the (1,0)-form $D z:=d z+p_{+}-i p_{3} z+p_{-} z^{2}$, where $z$ is a complex coordinate on the $\mathbb{C} P^{1}$ fiber and $\left(p_{3}, p_{+}, p_{-}\right)$is the $S U(2)$ part of the Levi-Civita connection on $\mathcal{M}$. The metric on $\mathcal{M}$ can be recovered from the Kähler-Einstein metric on $\mathcal{Z}_{\mathcal{M}}$ using

$$
d s_{\mathcal{Z}_{\mathcal{M}}}^{2}=\frac{1}{4}\left(e^{-2 K_{\mathcal{Z}_{\mathcal{M}}}}|\mathcal{C}|^{2}+\nu d s_{\mathcal{M}}^{2}\right)
$$

where $\nu$ is a numerical constant related to the curvature of the base manifold $\mathcal{M}$. Locally, any contact structure is trivial, so there exists an open covering $U_{i}$ of $\mathcal{Z}_{\mathcal{M}}$ and a local complex coordinate system $\left(\xi_{[i]}^{\Lambda}, \tilde{\xi}_{\Lambda}^{[i]}, \alpha_{[i]}\right)$ on $U_{i}$ such that the complex contact structure takes the Darboux form

$$
\mathcal{C}^{[i]}=d \alpha_{[i]}+\xi_{[i]}^{\Lambda} d \tilde{\xi}_{\Lambda}^{[i]}:=2 e^{\Phi_{[i]}} \frac{D z}{z} .
$$

The second equality defines the "contact potential" $\Phi_{[i]}$ in the patch $U_{i}$, a complex function on $\mathcal{Z}_{\mathcal{M}}$ holomorphic along the fiber. The contact potential in the patch $U_{i}$ is related to the Kähler potential $K_{\mathcal{Z}_{\mathcal{M}}}$ in the same patch via

$$
\mathcal{K}_{\mathcal{Z}_{\mathcal{M}}}^{[i]}=\log \frac{1+z \bar{z}}{|z|}+\Re\left[\Phi_{[i]}\left(x^{\mu}, z\right)\right] .
$$

Globally, the complex contact structure on $\mathcal{Z}_{\mathcal{M}}$ is determined by the complex contact transformations $S^{[i j]}$ between the Darboux coordinate system on the overlap $U_{i} \cap U_{j}$. These can be described, e.g., by providing holomorphic generating functions $S_{i j}\left(\xi_{[i]}^{\Lambda}, \tilde{\xi}_{\Lambda}^{[j]}, \alpha_{[j]}\right)$, subject to compatibility conditions on triple overlaps $U_{i} \cap U_{j} \cap U_{k}$, equivalence under local contact transformations on $U_{i}$ and $U_{j}$, and reality constraints. The quaternion-Kähler metric on $\mathcal{M}$ can then be extracted from these holomorphic data, by determining the contact twistor lines, i.e., expressing $\left(\xi_{\Lambda}, \tilde{\xi}^{\Lambda}, \alpha, \Phi\right)$ in some patch $U$ in terms of the coordinates $x^{\mu} \in \mathcal{M}$ on the base manifold and the complex coordinate $z \in \mathbb{C} P^{1}$ on the fiber. Plugging the solution into (5.2) allows one to extract 
the $S U(2)$ connection $p_{ \pm}, p_{3}$, the quaternionic 2 -forms and finally the metric on $\mathcal{M}$. More details on this construction can be found in [28,29]. It should be noted that these twistor techniques for quaternion-Kähler manifolds are related to the more standard twistor techniques for hyperkähler manifolds by the superconformal quotient construction $[23,26,27]$.

\subsubsection{On the twistor space of the tree-level universal hypermul-} tiplet. We now illustrate this construction in the case of the tree-level universal hypermultiplet moduli space. The twistor space $\mathcal{Z}_{\mathcal{M}_{\mathrm{UH}}}$ of the classical moduli space $\mathcal{M}_{\mathrm{UH}}$ can be nicely described group-theoretically as follows. Viewing the $\mathbb{C} P^{1}$ twistor fiber as $S^{2}=S U(2) / U(1)$, the fibration of $S U(2) / U(1)$ over $\mathcal{M}_{\mathrm{UH}}$ is such that the $S U(2)$ cancels $[63,76]$ :

$$
\mathcal{Z}_{\mathcal{M}_{\mathrm{UH}}}=\frac{S U(2)}{U(1)} \ltimes \frac{S U(2,1)}{S U(2) \times U(1)}=\frac{S U(2,1)}{U(1) \times U(1)} .
$$

The twistor space $\mathcal{Z}_{\mathcal{M}_{\mathrm{UH}}}$ is a complex three-dimensional contact manifold, with local coordinates $(\xi, \tilde{\xi}, \alpha)$. These coordinates parametrize the complexified Heisenberg group $N_{\mathbb{C}}$, or, equivalently, coordinates on the complex coset space $P_{\mathbb{C}} \backslash S L(3, \mathbb{C})$, where $P_{\mathbb{C}}$ is the complexification of the parabolic subgroup $P \subset S U(2,1)$ discussed in Appendix B and $S L(3, \mathbb{C})$ is the complexification of $S U(2,1)$. In terms of the coordinates $(\xi, \tilde{\xi}, \alpha)$ on $P_{\mathbb{C}} \backslash S L(3, \mathbb{C})$ the Kähler potential of $\mathcal{Z}_{\mathcal{M}_{\mathrm{UH}}}$ takes the following form [63]:

$$
K_{\mathcal{Z}_{\mathcal{M}_{\mathrm{UH}}}}=\frac{1}{2} \log \left[\left((\xi-\bar{\xi})^{2}+(\tilde{\xi}-\overline{\tilde{\xi}})^{2}\right)^{2}+4(\alpha-\bar{\alpha}+\bar{\xi} \tilde{\xi}-\xi \overline{\tilde{\xi}})^{2}\right]
$$

As mentioned above, the contact twistor lines for the unperturbed twistor space correspond to the change of variables that relate the coordinates $(\xi, \tilde{\xi}, \alpha)$ on $\mathcal{Z}_{\mathcal{M}_{\mathrm{UH}}}$ to the coordinates $x^{\mu}=\left\{e^{\phi}, \chi, \tilde{\chi}, \psi\right\}$ on the base $\mathcal{M}_{\mathrm{UH}}$ and the coordinate $z$ on the fiber $\mathbb{C} P^{1}=S U(2) / U(1)$. These twistor lines were obtained in [63]. In our notations they read (away from the north pole $z=0$ and south pole $z=\infty$ ) as

$$
\begin{aligned}
\xi & =-\sqrt{2} \chi+\frac{1}{\sqrt{2}} e^{-\phi}\left(z-z^{-1}\right), \\
\tilde{\xi} & =-\sqrt{2} \tilde{\chi}-\frac{i}{\sqrt{2}} e^{-\phi}\left(z+z^{-1}\right), \\
\alpha & =2 \psi-e^{-\phi}\left[z(\tilde{\chi}+i \chi)-z^{-1}(\tilde{\chi}-i \chi)\right] .
\end{aligned}
$$


Plugging these into (5.2) and (5.5), we find that the contact potential in this patch is simply

$$
e^{\Phi\left(x^{\mu}, z\right)}=e^{-2 \phi}
$$

in particular independent of $z$, and verify that (5.3) is satisfied. We further note that under an action of $S U(2,1)$, the contact potential and contact one-form transform as

$$
e^{\Phi} \longmapsto|C+D \mathcal{Z}|^{-2} e^{\Phi}, \quad \mathcal{C} \longmapsto(C+D \mathcal{Z})^{2} \mathcal{C}
$$

which ensure that the Kähler potential $K_{\mathcal{Z}_{\mathcal{M}_{\mathrm{UH}}}}$ transforms by a Kähler transformation, and that $S U(2,1)$ acts isometrically on both $\mathcal{Z}_{\mathcal{M}_{\mathrm{UH}}}$ and $\mathcal{M}_{\mathrm{UH}}$ itself.

\subsection{Quantum corrected hypermultiplet moduli spaces in type IIA}

Using these and related techniques, much progress has been achieved recently in understanding the hypermultiplet moduli space in type IIA string compactifications on a Calabi-Yau manifold $\mathcal{X}$. At the perturbative level, the metric on $\mathcal{M}_{\mathrm{H}}$ is believed to receive a one-loop correction, but no higher loop corrections $[17-20,77,78]$. For the universal hypermultiplet this was rigorously proven in [77]. The general form of the perturbative corrections can be inferred from compactifications of higher derivative couplings in 10 dimensions [17], or via an explicit string theory calculation in $D=4$ [20]. As a consequence, the contact potential on the twistor space must reduce at large volume, small coupling to

$$
e^{\Phi}=\frac{\tau_{2}^{2} V_{\mathcal{X}}}{2}+\frac{\chi_{E}}{192 \pi}+\ldots,
$$

where $\tau_{2}=1 / g_{s}$ is the 10-dimensional string coupling, $\chi_{\mathrm{E}}$ is the Euler number of $\mathcal{X}$ and $V_{\mathcal{X}}$ is the volume of $\mathcal{X}$ in string units. The complete perturbatively corrected metric corresponding to the contact potential (5.9) can be found in $[28,78]$. We note that in the corresponding expression in the type IIB hypermultiplet sector, there are additional contributions arising from $\alpha^{\prime}$-corrections and worldsheet instantons. However, due to the fact that the metric on the complex structure moduli space of $\mathcal{X}$ is insensitive to $\alpha^{\prime}$-effects, these corrections are absent in the type IIA expression (5.9). The corrections to the contact potential which are non-perturbative in $g_{s}$ are 
due to D2-brane and NS5-brane instantons [16]. Using S-duality and mirror symmetry, the form of the D2-brane instanton corrections was obtained in a series of works [27-31,79]. To first order away from the one-loop corrected metric, their contribution to the contact potential reads

$$
e^{\Phi_{(\mathrm{D} 2)}}=\frac{1}{4 \pi^{2}} \sum_{\gamma} n_{\gamma} \sum_{m>0} \frac{e^{-\phi}\left|Z_{\gamma}\right|}{m} K_{1}\left(8 \pi m e^{-\phi}\left|Z_{\gamma}\right|\right) e^{2 \pi i m \int_{\gamma} C_{(3)}}
$$

where $\gamma$ runs over the homology classes in $H^{3}(\mathcal{X}, \mathbb{Z}), Z_{\gamma}$ is the central charge associated to the cycle $\gamma, e^{\phi}$ is the $4 \mathrm{D}$ string coupling such that $e^{-2 \phi}=$ $\tau_{2}^{2} V_{\mathcal{X}} / 2, C_{(3)}$ is the Ramond-Ramond 3 -form and $n_{\gamma}$ is a numerical factor, which counts the number of BPS states in the homology class $\gamma$. NS5-brane contributions have been discussed in [34,79], but remain largely mysterious in general.

\subsection{On the contact potential and the Picard-Eisenstein series}

We now restrict to the case of type IIA string theory compactified on a rigid Calabi-Yau threefold, and propose that the Picard-Eisenstein series $\mathcal{E}_{s}(\phi, \chi, \tilde{\chi}, \psi)$, for a suitable value of the parameter $s$, controls the exact, quantum corrected metric on the universal hypermultiplet moduli space. As in [34] (see in particular Section 3.1), we shall restrict our attention to the contact potential $\Phi\left(x^{\mu}, z\right)$ on a certain holomorphic section ${ }^{22} z\left(x^{\mu}\right)$ of the twistor space $\mathcal{Z}_{\mathcal{M}_{\mathrm{UH}}}$. We also choose variables such that the action of $S U(2,1)$ on $\mathcal{M}_{\text {UH }}$ is the tree-level action (though it is no longer isometric in general), and look for a completion of $\Phi\left(x^{\mu}, z\left(x^{\mu}\right)\right)$, which reproduces the expected perturbative contributions. Determining the specific holomorphic section $z\left(x^{\mu}\right)$ and the exact twistor lines and hypermultiplet metric are important open problems which lie outside the scope of this work.

\footnotetext{
${ }^{22}$ In the presence of NS5-brane corrections, the contact potential is no longer constant along the fiber. The quaternion-Kähler metric on $\mathcal{M}=\mathcal{M}_{\mathrm{UH}}$ can nevertheless be described, in many different ways, in terms of a single real function $h\left(x^{\mu}\right)$ on $\mathcal{M}$ subject to a non-linear partial differential equation $[79,80]$. The latter can be identified with the Kähler potential $K_{\mathcal{Z}_{\mathcal{M}}}$ on any holomorphic section $z\left(x^{\mu}\right)$ of $\mathcal{Z}_{\mathcal{M}}$ [81]. Our proposal refers to a specific choice of holomorphic section, which we are not able to specify at this stage. We stress that this technical point plays no role at the level of our present analysis.
} 
Matching the powers of the dilaton, we then propose that, on the holomorphic section $z\left(x^{\mu}\right)$ introduced above, the contact potential for the quantum corrected metric on $\mathcal{M}_{\mathrm{UH}}$ is given by ${ }^{23}$

$$
e^{\Phi_{\text {exact }}\left(x^{\mu}, z\left(x^{\mu}\right)\right)}=\kappa e^{\phi} \mathcal{E}_{3 / 2}(\phi, \chi, \tilde{\chi}, \psi),
$$

where $\mathcal{E}_{s}$ is the Picard-Eisenstein series (3.2), and $\kappa$ is an adjustable numerical constant. Using the Fourier expansion (1.18), we see that (5.11) predicts

$$
e^{\Phi_{\text {exact }}}=4 \zeta_{\mathbb{Q}(i)}(3 / 2) \kappa\left(e^{-2 \phi}+\frac{\mathfrak{Z}(1 / 2)}{\mathfrak{Z}(3 / 2)}\right)+e^{\Phi_{(\mathrm{A})}}+e^{\Phi_{(\mathrm{NA})}},
$$

where the last two terms correspond to the Abelian and non-Abelian parts of the Fourier expansion, respectively. The two constant terms have the same dependence on the dilaton $e^{\phi}$ as the two perturbative contributions in (5.9). We thus want to identify the second constant term at $s=3 / 2$ with the one-loop coefficient $\chi_{\mathrm{E}} / 192 \pi$ in (5.9). Here we run into a problem since for $s=3 / 2$ we find

$$
\frac{\mathfrak{Z}(1 / 2)}{\mathfrak{Z}(3 / 2)} \approx-2.32607
$$

implying that matching with the physical one-loop term requires $\chi_{\mathrm{E}} \sim$ -1403.05 , a negative, non-integer number. This contradicts the fact that $\chi_{\mathrm{E}}=2 h_{1,1} \in 2 \mathbb{N}$ for a rigid Calabi-Yau threefold. Hence, the value of the one-loop coefficient predicted by the second constant term in the Eisenstein series is not physically viable. While this invalidates the proposal that the principal Eisenstein series $\mathcal{E}_{s}$ describes the exact universal hypermultiplet metric, it does not necessarily ruin the idea that the Picard modular group controls that metric. In the concluding Section 6 , we speculate that automorphic forms attached to the quaternionic discrete series of $S U(2,1, \mathbb{Z}[i])$ may be relevant. We proceed with our current proposal however, as the form of the non-Abelian Fourier expansion is largely independent of the details of the automorphic form under consideration. In particular, we show next that the form of the Abelian and non-Abelian contributions to the Fourier expansion of $\mathcal{E}_{3 / 2}(\phi, \chi, \tilde{\chi}, \psi)$ agrees with the expected form of D2-brane and NS5-brane instanton contributions, respectively.

\footnotetext{
${ }^{23}$ Due to the different power of $e^{\phi}$, the contact potential $\Phi_{\text {exact }}$ appears to transform differently from its tree-level counterpart (5.8); this is not a fatal flaw however, since the locus $z\left(x^{\mu}\right)$ is in general not fixed by the action of the Picard modular group.
} 
5.3.1. D2-brane instantons. The Abelian contribution (4.65) at $s=$ $3 / 2$ becomes

$$
\begin{aligned}
e^{\Phi_{(\mathrm{A})}}= & \frac{2 \kappa \zeta_{\mathbb{Q}(i)}(3 / 2) e^{-\phi}}{\mathfrak{Z}(3 / 2)} \sum_{\left(\ell_{1}, \ell_{2}\right) \in \mathbb{Z}^{2}}^{\prime} \mu_{3 / 2}\left(\ell_{1}, \ell_{2}\right)\left[\ell_{1}^{2}+\ell_{2}^{2}\right]^{1 / 2} \\
& \times K_{1}\left(2 \pi e^{-\phi} \sqrt{\ell_{1}^{2}+\ell_{2}^{2}}\right) e^{-2 \pi i\left(\ell_{1} \chi+\ell_{2} \tilde{\chi}\right)}
\end{aligned}
$$

where the summation measure $\mu_{3 / 2}\left(\ell_{1}, \ell_{2}\right)$ is given in (4.68). In the weakcoupling limit $e^{\phi} \rightarrow 0$ we may use the asymptotic expansion of the modified Bessel function at large $x$,

$$
K_{t}(x) \sim \sqrt{\frac{\pi}{2 x}} e^{-x} \sum_{n \geq 0} \frac{\Gamma\left(t+n+\frac{1}{2}\right)}{\Gamma(n+1) \Gamma\left(t-n+\frac{1}{2}\right)}(2 x)^{-n}
$$

to approximate

$$
e^{\Phi_{(\mathrm{A})}} \sim \frac{\kappa \zeta_{\mathbb{Q}(i)}(3 / 2)}{\mathfrak{Z}(3 / 2)} e^{-\phi / 2} \sum_{\left(\ell_{1}, \ell_{2}\right) \in \mathbb{Z}^{2}}^{\prime} \mu_{3 / 2}\left(\ell_{1}, \ell_{2}\right)\left(\ell_{1}^{2}+\ell_{2}^{2}\right)^{1 / 4} e^{-2 \pi S_{\ell_{1}, \ell_{2}}}\left[1+\mathcal{O}\left(e^{\phi}\right)\right] .
$$

We thus find that $e^{\Phi_{(\mathrm{A})}}$ exhibits exponentially suppressed corrections in the limit $e^{\phi} \rightarrow 0$, weighted by the instanton action

$$
S_{\ell_{1}, \ell_{2}}=e^{-\phi} \sqrt{\ell_{1}^{2}+\ell_{2}^{2}}+i\left(\ell_{1} \chi+\ell_{2} \tilde{\chi}\right) .
$$

This is recognized as the action for Euclidean D2-branes wrapping special Lagrangian 3 -cycles in the homology class $\ell_{1} \mathcal{A}+\ell_{2} \mathcal{B} \in H_{3}(\mathcal{X}, \mathbb{Z})$, where $(\mathcal{A}, \mathcal{B})$ provides an integral symplectic basis of $H_{3}(\mathcal{X}, \mathbb{Z})$. To see this, we note that generally the instanton action for D2-branes wrapping a special Lagrangian submanifold in the homology class $\gamma \in H_{3}(X, \mathbb{Z})$ inside a CalabiYau threefold $\mathcal{X}$ is given by

$$
S_{\gamma}=\frac{1}{g_{s}}\left|\int_{\gamma} \Omega\right|+i \int_{\gamma} C_{(3)}
$$

where $g_{s}$ is the 10-dimensional string coupling, $\Omega \in H_{3,0}(X)$ is the holomorphic three-form and $C_{(3)} \in H^{3}(X, \mathbb{R}) / H^{3}(X, \mathbb{Z})$ is the RR three-form. The real part of the action can further be written in terms of the central charge $Z_{\gamma}=e^{K / 2} \int_{\gamma} \Omega$ as $\Re\left(S_{\gamma}\right)=e^{-K / 2}\left|Z_{\gamma}\right| / g_{s}$, where $K=-\log \int_{X} \Omega \wedge \bar{\Omega}$ 
is the Kähler potential of the complex structure moduli space. Noting that $K=-\log V_{\mathcal{X}}$ we then find

$$
S_{\gamma}=e^{-\phi}\left|Z_{\gamma}\right|+i \int_{\gamma} C_{(3)},
$$

where we defined the four-dimensional dilaton by $e^{\phi}:=V_{\mathcal{X}}^{-1 / 2} g_{s}$. Restricting to a rigid Calabi-Yau threefold $\mathcal{X}$, we recall from Section 1 that the prepotential is $F=\tau X / 2$ with $\tau$ being the period "matrix" $\int_{\mathcal{B}} \Omega / \int_{\mathcal{A}} \Omega$. In this case the D2-brane wraps a three-cycle in the homology class $\gamma=$ $\ell_{1} \mathcal{A}+\ell_{2} \mathcal{B} \in H_{3}(\mathcal{X}, \mathbb{Z})$, which gives $Z_{\gamma}=\left(\ell_{1}+\tau \ell_{2}\right) / \sqrt{\Im \tau}$, so the instanton action reduces to

$$
S_{\ell_{1}, \ell_{2}}(\tau)=e^{-\phi} \frac{\left|\ell_{1}+\tau \ell_{2}\right|}{\sqrt{\Im \tau}}+i \int_{\ell_{1} \mathcal{A}+\ell_{2} \mathcal{B}} C_{(3)}
$$

Further setting $\tau=i$, which is the relevant value for our analysis, and using Equation (1.5) for the periods of the Ramond-Ramond three-form $C_{(3)}$, this action indeed coincides with the instanton action (5.17) predicted from $S U(2,1 ; \mathbb{Z}[i])$-invariance. Thus, we may conclude that the Abelian term (5.14) in the Fourier expansion agrees with the general form of D-instanton corrections in (5.10) upon restricting to a rigid Calabi-Yau threefold, which admits complex multiplication by $\mathbb{Z}[i]$.

The infinite series within the brackets in (5.16) should, in the spirit of [5], arise from perturbative contributions around the instanton background. The summation measure is given by specifying (4.68) to $s=3 / 2$,

$$
\mu_{3 / 2}\left(\ell_{1}, \ell_{2}\right)=\sum_{\omega_{3}^{\prime} \mid \Lambda}\left|\omega_{3}^{\prime}\right|^{-1} \sum_{z \mid \frac{\Lambda}{\omega_{3}^{\prime}}}|z|^{-2},
$$

where we recall that $\Lambda=\ell_{2}-i \ell_{1}$ is a complex combination of the electric and magnetic charges $\left(\ell_{1}, \ell_{2}\right)$. The instanton measure $\mu_{3 / 2}\left(\ell_{1}, \ell_{2}\right)$ should count the degeneracy of Euclidean D2-branes in the homology class $\ell_{1} \mathcal{A}+\ell_{2} \mathcal{B} \in$ $H_{3}(\mathcal{X}, \mathbb{Z})$. For D2-instantons with $A$-type charge only, i.e., $\ell_{2}=0$, and such that $\ell:=\ell_{1}$ is a product of inert primes (those of the form $p=4 n+3$, see Appendix A), the first sum collapses to $\omega_{3}^{\prime}=1$ and the instanton measure reduces to

$$
\mu_{3 / 2}(\ell, 0)=\sum_{z \mid \ell}|z|^{-2}
$$


This reproduces the instanton measure found on the basis of $S L(2, \mathbb{Z})$ invariance in [29-31,82], which by analogy with $[5,9,10]$ should count ways of splitting a marginal bound state into smaller constituents. However, it is possible that $\ell$ be prime over the integers but factorizable over the Gaussian integers, e.g., $2=-i(1+i)^{2}$ or $5=(2+i)(2-i)$, in which case the measure (5.21) involves additional contributions compared to [29-31,82]. This novel feature of compactifications on rigid Calabi-Yau manifolds is an interesting prediction of $S U(2,1 ; \mathbb{Z}[i])$-invariance which deserves further investigation.

5.3.2. NS5-brane instantons. As mentioned above, the non-Abelian term $e^{\Phi_{(\mathrm{NA})}}$ may be interpreted as NS5-brane instanton contributions. Although we have not been able to extract the coefficients $C_{r, k, \ell}^{(\mathrm{NA})}(s)$ in the non-Abelian Fourier expansion (4.10), we can still extract the instanton action by taking the semiclassical limit. This corresponds to the asymptotic behavior of (4.10) in the limit $y \rightarrow \infty$, or, equivalently, to the saddle point approximation of the $t$-integral in (4.83) as analyzed in Section 4.6. Expanding the Whittaker function around $x=\infty$ yields

$$
\begin{aligned}
& W_{k, m}(x) \sim e^{-x / 2} x^{k} \sum_{n \geq 0} \frac{\Gamma\left(m-k+n+\frac{1}{2}\right) \Gamma\left(m+k+\frac{1}{2}\right)}{\Gamma(n+1) \Gamma\left(m-k+\frac{1}{2}\right) \Gamma\left(m+k-n+\frac{1}{2}\right)} x^{-n} \\
& \quad \sim e^{-x / 2} x^{k}[1+\mathcal{O}(1 / x)] .
\end{aligned}
$$

Implementing this in (4.10) and extracting the leading $r=0$ term, we deduce that the leading order contribution to $e^{\Phi_{(\mathrm{NA})}}$ is given by

$$
e^{\Phi_{(\mathrm{NA})}} \sim e^{\phi} \sum_{k \in \mathbb{Z}}^{\prime} \sum_{\ell=0}^{4|k|-1} \sum_{n \in \mathbb{Z}+\frac{\ell}{4|k|}} C_{r, k, \ell}|k|^{-s} e^{-2 \pi S_{k, q}}\left[1+\mathcal{O}\left(e^{2 \phi}\right)\right]
$$

where we have defined

$$
S_{k, q}=|k| e^{-2 \phi}+2|k|(\tilde{\chi}-n)^{2}-i q \chi+2 i k(\psi+\chi \tilde{\chi}) .
$$

This reproduces the Euclidean action of $k$ NS5-branes bound to $q:=4 n k$ D2branes. Note that even in the absence of D2-brane instanton contributions, $q=0$, the real part of the action receives a contribution from the background Ramond-Ramond flux $\tilde{\chi}$, as found previously in [79]. For vanishing $\tilde{\chi}$, this 
reduces to the pure NS5-brane instanton action of [16]:

$$
S_{k}=|k| e^{-2 \phi}+2 i k \psi
$$

It should be emphasized that the result (5.24) displays the contribution from $A$-type D2-brane instantons only. The $B$-type D2-branes could be exposed by choosing the alternative polarization displayed in (4.7), but then the $A$-type D2-brane effects are not visible. This is in contrast to the situation in [34], where the appearance of an extra summation in the non-Abelian term made it possible to expose the $\mathrm{D}(-1)$, D5 and NS5-brane effects simultaneously. ${ }^{24}$

Finally, we observe that the asymptotic expansion of the Whittaker function predicts an infinite series of perturbative corrections around the NS5-brane instanton background. This is in marked contrast to the case of type IIA Euclidean NS5-branes wrapping $K 3 \times T^{2}$, where the perturbative corrections around the instanton background truncate at one loop [84].

\section{Conclusions}

In this work we postulated that quantum corrections to the hypermultiplet moduli space in type IIA string theory compactified on a rigid CalabiYau threefold with complex multiplication by $\mathbb{Z}[i]$ are controlled by the Picard modular group $S U(2,1 ; \mathbb{Z}[i])$. We investigated the consequences of this assumption for the simplest automorphic form, the Eisenstein series (1.14). Despite a serious discrepancy with the sign of the one-loop term, the fact that we were led to D2- and NS5-brane instanton corrections with the correct classical action provides support for our postulate. In the case of D2-brane instantons, the prediction of the Eisenstein series (5.14) is in fact in full agreement with the general form predicted in $[29,31,82]$, though one could argue that it is largely a consequence of the Laplace equation (1.13). The instanton measure (5.22) is also similar to the dilogarithm sum found in [29], with additional refinements when the charges include non-inert prime factors. It would be interesting to compare the instanton summation measure with the generalized Donaldson-Thomas invariants of rigid Calabi-Yau manifolds.

\footnotetext{
${ }^{24}$ We note that the presence of an extra "theta-angle" in the NS5-brane instanton action of [34], compared to our result (5.25), is related to the fact that the spherical vector $f_{K}$ in the principal series of $S L(3, \mathbb{R})$ displays a cubic phase factor [83] which is absent in the corresponding spherical vector for $S U(2,1)$ [63].
} 
While the sign of the one-loop term invalidates our proposal that the Eisenstein series (1.14) governs the exact metric on the hypermultiplet moduli space, and so forbids us to expect a detailed agreement between our summation measure and the generalized Donaldson-Thomas invariants, we do not think that it ruins the basic postulate that the Picard modular group $S U(2,1 ; \mathbb{Z}[i])$ should act isometrically on the exact universal hypermultiplet moduli space. Rather, we take it as an incentive to construct a more sophisticated automorphic form, which would produce the correct one-loop term, as well as produce a non-trivial dependence on the coordinate $z$ on the twistor fiber $\mathbb{C} P^{1}$, which is generally expected when all isometries are broken. In fact, since the twistor space is known to be described by holomorphic contact transformations, it is natural to expect that automorphic forms attached to the quaternionic discrete series of $S U(2,1)$ should be relevant. Indeed, these forms can be lifted to sections of a certain complex line bundle on the twistor space $\mathcal{Z}_{\mathcal{M}_{\mathrm{UH}}}=S U(2,1) /(U(1) \times U(1))[63,85]$. It is challenging to construct such automorphic forms explicitly, and adapt the analysis in $[33]$ to produce a manifestly $S U(2,1, \mathbb{Z}[i])$-invariant description of the twistor space. We anticipate, however, that the resulting instanton corrections will be qualitatively similar to the ones considered here, although the summation measure will certainly be quite different.

In this work we have concentrated exclusively on rigid Calabi-Yau threefolds whose intermediate Jacobian $J(\mathcal{X})$ admits complex multiplication by $\mathbb{Z}[i]$. It is interesting to ask how our construction may generalize to other values of the period matrix $\tau$. When $J(\mathcal{X})$ admits complex multiplication by the ring of integers $\mathcal{O}_{d}$ in the imaginary quadratic number field $\mathbb{Q}(\sqrt{-d}), d>0$, it is natural to conjecture that the relevant arithmetic subgroup of $S U(2,1)$ would be $S U\left(2,1 ; \mathcal{O}_{d}\right)$. For example, choosing $\tau=$ $(1+i \sqrt{3}) / 2:=\omega$ should correspond to the "Picard-Eisenstein" modular group $S U(2,1 ; \mathbb{Z}[\omega])$, where $\mathbb{Z}[\omega]$ are the Eisenstein integers, corresponding to the ring of integers $\mathcal{O}_{3}=\mathbb{Z}[\omega]$ in $\mathbb{Q}(\sqrt{-3})[86]$. In contrast to the $\tau=i$ case, it is interesting to note that $S U(2,1 ; \mathbb{Z}[\omega])$ does not contain the "rotation" generator $R$ in (1.10) [87]. Indeed, one does not generally expect the full electric-magnetic duality group to be a quantum symmetry, but rather its subgroup generated by monodromies in the moduli space of complex structures (which is non-existent in the case of rigid Calabi-Yau threefolds).

From a purely mathematical point of view, we have provided several explicit constructions of an automorphic form for the Picard modular group attached to the principal continuous series of $S U(2,1)$. In addition, we analyzed its Abelian and non-Abelian Fourier expansion in detail and found 
evidence for its functional equation. Sums over Gaussian divisors and Dirichlet $L$-series for the Gauss field play central roles in the analysis. We expect that our results will be useful in subsequent investigations of automorphic forms for various types of Picard groups.

\section{Acknowledgments}

We are grateful to Sergei Alexandrov, Guillaume Bossard, Claudia Colonnello, Gabor Francsics, Ulf Gran, Nick Halmagyi, Peter D. Lax, Jakob Palmkvist, Ulf Persson, Christoffer Petersson, Per Salberger, Frank Saueressig, Jan Stienstra, Stefan Theisen, Stefan Vandoren, Pierre Vanhove, Niclas Wyllard, Don Zagier and Genkai Zhang for helpful discussions and correspondence. We also thank Jakob Palmkvist and Christoffer Petersson for many useful comments on an early version of this manuscript.

A.K. is a Research Associate of the Fonds de la Recherche ScientifiqueFNRS, Belgium. This work has been supported in part by IISN-Belgium (conventions 4.4511.06, 4.4505.86 and 4.4514.08) and by the Belgian Federal Science Policy Office through the Interuniversity Attraction Pole P6/11.

\section{Appendix A. Dirichlet series and Gaussian integers}

In this appendix, we collect for the reader's convenience some standard facts about Dirichlet series and Gaussian integers.

\section{A.1. Euler products and Dirichlet series}

A series $a(n)$ for $n \in \mathbb{N}$ is called multiplicative if and only if $a\left(n_{1} n_{2}\right)=$ $a\left(n_{1}\right) a\left(n_{2}\right)$ whenever $n_{1}$ and $n_{2}$ are coprime [88]. The associated Dirichlet series

$$
L(a, s)=\sum_{n>0} a(n) n^{-s}
$$

constructed from a multiplicative $a(n)$ can be recast as an Euler product over the primes $(p>1)$

$$
L(a, s)=\prod_{p \text { prime }} P(p, s),
$$


where

$$
P(p, s)=\sum_{k \geq 0} a\left(p^{k}\right) p^{-k s}
$$

As an example consider the multiplicative series (4.43). One finds

$$
P(2, s)=\sum_{k \geq 0}\left(2^{1-s}\right)^{k}=\frac{1}{1-2^{1-s}}
$$

and for Pythagorean primes $p=1 \bmod 4$

$$
P(p, s)=\sum_{k \geq 0}\left(p^{1-s}\right)^{k}+\frac{p-1}{1-s} \partial_{p} \sum_{k \geq 0}\left(p^{1-s}\right)^{k}=\frac{1-p^{-s}}{\left(1-p^{1-s}\right)^{2}}
$$

For primes of the form $p=3 \bmod 4$ one has

$$
\text { (A.6) } P(p, s)=\sum_{k \geq 0}\left(p^{2-2 s}\right)^{k}+p^{-s} \sum_{k \geq 0}\left(p^{2-2 s}\right)^{k}=\frac{1+p^{-s}}{\left(1-p^{1-s}\right)\left(1+p^{1-s}\right)} \text {, }
$$

whence one recovers (4.54).

\section{A.2. Structure of Gaussian primes}

The ring of Gaussian integers $\mathbb{Z}[i]$ forms a principal ideal domain $[74,89]$, i.e., every element admits a unique prime factorization up to migration of the four units $\pm 1, \pm i$. We will use the notation $g$ for Gaussian primes and $p$ for standard (rational) primes. Gaussian prime numbers $g=a+i b \in \mathbb{Z}[i]$ fall under three different cases, called ramified, inert and split.

(i) The first case consists solely of $g=1+i$. Since $2=-i(1+i)^{2}$, this implies that $p=2$, despite being prime in $\mathbb{Z}$, is no longer prime in $\mathbb{Z}[i]$. The rational prime $p=2$ is said to be ramified over the Gaussian integers.

(ii) Inert primes are of the form $g=4 n+3$ for some $n \in \mathbb{N}$ such that $4 n+3$ is prime in $\mathbb{Z}$. The name inert indicates that such integers are prime both over $\mathbb{Z}$ and $\mathbb{Z}[i]$.

(iii) Split primes come in complex conjugate pairs $g$ and $\bar{g}$. Such $g=a+$ $i b, b \neq 0$ are prime if and only if $p:=g \bar{g}=a^{2}+b^{2}$ is a standard prime in $\mathbb{Z}$ and $p>2$. By Fermat's theorem on the sums of squares $p$ must be of the form $p=4 n+1$ for some $n \in \mathbb{N}$, i.e., a Pythagorean prime. 


\section{Appendix B. Spherical vector and $p$-adic Eisenstein series}

Automorphic forms can be constructed quite generally using adelic methods, as explained for the layman, e.g., in [60-62]. In this appendix, we apply this method to recover the Eisenstein series $\mathcal{E}_{s}(\phi, \lambda, \gamma)$ for the Picard modular group. This alternative approach also sheds light on the relation between the quadratic constraint (3.3) and the representation-theoretic structure underlying the Eisenstein series. This appendix may be viewed as an automorphic extension of the results in Section 2 of [63].

\section{B.1. Formal construction}

In general, to construct an automorphic form $\Psi$ on $G / K$, invariant under a discrete subgroup $G(\mathbb{Z}) \subset G$, we require three ingredients: (1) a $K$-invariant spherical vector $f_{K} \in \mathcal{H}$ ( $\mathcal{H}$ being a Hilbert space of square integrable functions), (2) a linear representation $\rho$ of $G$ acting on $\mathcal{H}$, and (3) a $G(\mathbb{Z})$ invariant distribution $f_{\mathbb{Z}} \in \mathcal{H}^{\star}$ in the dual space of $\mathcal{H}$. Using the natural pairing $\langle$,$\rangle between \mathcal{H}$ and $\mathcal{H}^{\star}$, the automorphic form $\Psi$ can then be defined formally as

$$
\Psi(g):=\left\langle f_{\mathbb{Z}}, \rho(g) \cdot f_{K}\right\rangle,
$$

with $g \in G$. By virtue of the Iwasawa decomposition,

$$
G=N A K,
$$

an arbitrary group element $g \in G$ splits as $g=n a k:=\mathcal{V} k$, and, since $f_{K}$ is $K$-invariant, $\Psi$ simplifies to

$$
\Psi(\mathcal{V})=\left\langle f_{\mathbb{Z}}, \rho(\mathcal{V}) \cdot f_{K}\right\rangle .
$$

The coset representative $\mathcal{V} \in G / K$ transforms by $k^{-1} \in K$ from the right and $\gamma \in G(\mathbb{Z})$ from the left,

$$
\mathcal{V} \longmapsto \gamma \mathcal{V} k^{-1} .
$$

From the point of view of $\Psi(\mathcal{V})$ the right action by $k^{-1}$ on $\rho(\mathcal{V})$ becomes a left action on $f_{K}$, which is invariant by definition, and the left action of $\gamma$ becomes a right action on $f_{\mathbb{Z}}$, which is also invariant. Hence, $\Psi(\mathcal{V})$ is by construction a function on the double quotient $G(\mathbb{Z}) \backslash G / K$ as desired. 
Although very appealing, this method is often unpractical due to the difficulty of obtaining the invariant distribution. Adelic methods offer a powerful way to obtain $f_{\mathbb{Z}}$, by reducing this problem to that of finding the $p$-adic spherical vector $f_{p}$ for all primes $p$ (see $[90,91]$ for an introduction to $p$-adic numbers, and [60-62] for illustrations of the adelic method). The distribution $f_{\mathbb{Z}}(x)$ is then obtained as the product of $f_{p}(x)$ over all prime numbers of the given number field. For our purposes, $p$ runs over the Gaussian primes and we will denote it by $g$ in accordance with Appendix A. Nevertheless, we will refer to the approach as the $p$-adic approach. The function $\Psi$ then can be rewritten formally as

$$
\Psi(\mathcal{V})=\sum_{\vec{x} \in \mathbb{Q}(i)^{n}}^{\prime} \rho(\mathcal{V}) \cdot\left[\prod_{g \text { prime }} f_{g}(\vec{x})\right]
$$

where $\vec{x}$ is a vector of rational Gaussian numbers in $\mathbb{Q}(i)^{n}$, the product runs over all Gaussian prime numbers $g$ including the "place at infinity" $g=\infty$, and we defined $f_{\infty}=f_{K}$. Note that we restrict to the case where $\mathcal{V} \in G(\mathbb{R})$, such that $\rho(\mathcal{V})$ acts only on $f_{K}$, but it is natural to extend (B.5) to the case where $\mathcal{V}$ is an element of the adele group $G(\mathbb{A})$, in which case $\rho(\mathcal{V})$ acts on all the $f_{g}$ 's as well. We shall now see that the Eisenstein series $\mathcal{E}_{s}(\phi, \lambda, \gamma)$, constructed in Section 3.1, can indeed be obtained from this adelic point of view.

\section{B.2. Real and $p$-Adic spherical vector}

To reproduce the Picard-Eisenstein series (3.2) by this method, we consider the principal continuous series representation of $S U(2,1)$, induced from the Heisenberg parabolic $P$ whose Lie algebra consists of the non-positive grade part of the 5-grading (2.8):

$$
\mathfrak{p}=\mathfrak{g}_{-2} \oplus \mathfrak{g}_{-1} \oplus \mathfrak{g}_{0} \subset \mathfrak{s u}(2,1)
$$

The parabolic group $P$ thus corresponds to the subgroup of lower-triangular matrices,

$$
P=\left\{\left(\begin{array}{ccc}
t_{1} & & \\
* & t_{2} & \\
* & * & t_{3}
\end{array}\right) \in S U(2,1): t_{1} t_{2} t_{3}=1\right\}
$$


The coset space $P \backslash S U(2,1)$ is isomorphic to the Heisenberg group $N$, and can be parameterized as follows:

$$
n=e^{x X_{1}+\tilde{x} \tilde{X}_{1}+2 y X_{2}}=\left(\begin{array}{ccc}
1 & i \bar{C}_{2} & C_{1} \\
& 1 & C_{2} \\
& & 1
\end{array}\right):=\left(\begin{array}{c}
\vec{r}_{1} \\
\vec{r}_{2} \\
\vec{r}_{3}
\end{array}\right) \in N
$$

where

$$
C_{1}:=2 y+\frac{i}{2}\left|C_{2}\right|^{2}, \quad C_{2}:=x+\tilde{x}+i(\tilde{x}-x)
$$

satisfy the quadratic relation

$$
\left|C_{2}\right|^{2}-2 \Im\left(C_{1}\right)=0
$$

and the last equality in (B.8) defines the row vectors $\vec{r}_{i}$ of the Heisenberg group element.

The coset space $N=P \backslash S U(2,1)$ admits an action of $g \in S U(2,1)$ by multiplication from the right, followed by a compensating action by $p(g) \in P$ from the left so as to restore the upper triangular gauge (B.8). The principal continuous series representation consists of functions $f(x, \tilde{x}, y)$ on $N$ transforming by the character $\chi_{s}(p(g))$ under the action of $g$, where

$$
\chi_{s}(p):=t_{1}^{-2 s}, \quad p=\left(\begin{array}{ccc}
t_{1} & & \\
* & t_{2} & \\
* & * & t_{3}
\end{array}\right) \in P .
$$

The spherical vector $f_{K}$ can be obtained straightforwardly as follows [63], while the compensating left-action of $P$ on the second and third rows, $\vec{r}_{2}$ and $\vec{r}_{3}$, of $n$ is quite complicated, the action on the first row $\vec{r}_{1}$ is very simple: $p \in P$ simply modifies $\vec{r}_{1}$ by an overall factor of $t_{1}$. Moreover, the action of $k \in S U(2) \times U(1)$ leaves invariant the (complex) norms of the rows $\vec{r}_{i}$. The spherical vector $f_{K}$ can therefore be obtained by raising the norm of the first row $\vec{r}_{1}$ of $n$ to the appropriate power of $s[63]:^{25}$

$$
\begin{aligned}
f_{K}(x, \tilde{x}, y) & :=\left|\vec{r}_{1}\right|^{-2 s}=\left(1+\left|C_{1}\right|^{2}+\left|C_{2}\right|^{2}\right)^{-s} \\
& =\left(1+2\left(x^{2}+\tilde{x}^{2}\right)+4 y^{2}+\left(x^{2}+\tilde{x}^{2}\right)^{2}\right)^{-s} .
\end{aligned}
$$

This object is indeed invariant under $S U(2) \times U(1)$, since the right action of $k$ on $n$ is a "rotation" that preserves the norm, while the compensating

\footnotetext{
${ }^{25}$ See also [83] for a similar construction in the context of $S L(3, \mathbb{R})$.
} 
left action of $p$ merely modifies $f_{K}$ by an overall factor $t_{1}^{2 s}$, which in turn is canceled against the character $\chi_{s}(p)=t_{1}^{-2 s}$ which is present since $f_{K}$ is in the principal series.

The next step is to compute the action of $\rho(\mathcal{V})$ on $f_{K}$. Following the prescription above, this can be done by first computing $n \cdot \mathcal{V}=p_{0} \cdot n^{\prime}$, with

$$
\begin{aligned}
p_{0} & =\left(\begin{array}{ccc}
e^{-\phi} & & \\
& 1 & \\
& & e^{\phi}
\end{array}\right) \in P, \\
n^{\prime} & =\left(\begin{array}{ccc}
1 & i e^{\phi}\left(\bar{\lambda}+\bar{C}_{2}\right) & e^{2 \phi}\left(\gamma+i \bar{C}_{2} \lambda+C_{1}\right) \\
& 1 & e^{\phi}\left(\lambda+C_{2}\right) \\
& & 1
\end{array}\right) \in P \backslash S U(2,1) .
\end{aligned}
$$

Applying this to the spherical vector $f_{K}(x, \tilde{x}, y)=f_{K}(n)$ yields

$$
\rho(\mathcal{V}) \cdot f_{K}(n)=f_{K}(n \mathcal{V})=f_{K}\left(p_{0} n^{\prime}\right)=\chi_{s}\left(p_{0}\right) f_{K}\left(n^{\prime}\right)=e^{2 s \phi}\left|\vec{r}_{1}^{\prime}\right|^{-2 s}
$$

which may be written explicitly in the form

$$
\rho(\mathcal{V}) \cdot f_{K}\left(C_{1}, C_{2}\right)=e^{-2 s \phi}\left(\left|\bar{C}_{1}-i C_{2} \bar{\lambda}+\bar{\gamma}\right|^{2}+e^{-2 \phi}\left|C_{2}+\lambda\right|^{2}+e^{-4 \phi}\right)^{-s} .
$$

The $p$-adic spherical vector $f_{p}\left(C_{1}, C_{2}\right)$ can now be found by replacing the Euclidean norm $|\cdot|$ appearing in the real spherical vector $f_{K}$ by its counterpart over the $p$-adic Gaussian numbers ${ }^{26}$ (see, e.g., [92])

$$
|z|_{g}^{\mathbb{Q}(i)}:=|g|^{-k}, \quad z \in \mathbb{Q}(i)
$$

for any Gaussian prime $g$, with $k \in \mathbb{Z}$ being the maximum power of $g$ appearing in the prime factorization of $z$ in Gaussian primes. The $p$-adic spherical vector for a Gaussian prime $g$ is then given by

$$
f_{g}\left(C_{1}, C_{2}\right):=\left[\left|\vec{r}_{1}\right|_{g}^{\mathbb{Q}(i)}\right]^{-2 s}=\max \left(1,\left|\bar{C}_{1}\right|_{g}^{\mathbb{Q}(i)},\left|C_{2}\right|_{g}^{\mathbb{Q}(i)}\right)^{-2 s}
$$

\footnotetext{
${ }^{26}$ Note that with this definition, $|z|_{g}^{\mathbb{Q}(i)}$ is not invariant under complex conjugation, as can be seen easily by taking $z$ to be a split prime. The definition of the $p$-adic norm in [91] differs from the one we use. It is invariant under complex conjugation but misses other desirable properties; in particular it does not reproduce Equation (B.19) correctly.
} 


\section{B.3. Product over primes}

The automorphic form $\Psi(\mathcal{V})$ in this representation now reads

$$
\Psi(\mathcal{V})=\sum_{\substack{\left(C_{1}, C_{2}\right) \in \mathbb{Q}(i) \\\left|C_{2}\right|^{2}-2 \Im\left(C_{1}\right)=0}}^{\prime} \rho(\mathcal{V}) \cdot\left[\prod_{g<\infty} f_{g}\left(C_{1}, C_{2}\right)\right] f_{K}\left(C_{1}, C_{2}\right)
$$

Next we must evaluate the infinite product over Gaussian prime numbers $g$. To this end we split the rational variables $C_{1}$ and $C_{2}$ in the following way:

$$
C_{1}=\frac{\omega_{1}}{\omega_{3}}, \quad C_{2}=\frac{i \bar{\omega}_{2}}{\bar{\omega}_{3}}
$$

with $\omega_{j} \in \mathbb{Z}[i]$, for $j=1,2,3, \operatorname{gcd}\left(\omega_{1}, \omega_{2}, \omega_{3}\right)=1 .{ }^{27}$ Using the definition (B.15) we can explicitly evaluate the infinite product over primes in (B.17) as

$$
\prod_{g<\infty} \max \left(1,\left|\frac{\bar{\omega}_{1}}{\bar{\omega}_{3}}\right|_{g}^{\mathbb{Q}(i)},\left|\frac{\bar{\omega}_{2}}{\bar{\omega}_{3}}\right|_{g}^{\mathbb{Q}(i)}\right)^{-2 s}=\left|\omega_{3}\right|^{-2 s} .
$$

Multiplying the constraint (B.10) further by a factor of $\left|\omega_{3}\right|^{2}$ one obtains

$$
\left|\omega_{3}\right|^{2}\left(\left|C_{2}\right|^{2}-2 \Im\left(C_{1}\right)\right)=\left|\omega_{2}\right|^{2}-2 \Im\left(\omega_{1} \bar{\omega}_{3}\right)=\vec{\omega}^{\dagger} \cdot \eta \cdot \vec{\omega}=0 .
$$

Combining Equations (B.17), (B.19) and (B.20) and adding the contribution at $C_{1}=C_{2}=\infty$ (i.e., $\omega_{3}=0$ ) then yields the final form of $\Psi(\mathcal{V})$ :

$$
\begin{aligned}
& \Psi(\mathcal{V})=\sum_{\substack{\vec{\omega} \in \mathbb{Z}[i]^{3},\left|\omega_{2}\right|^{2}-2 \Im\left(\omega_{1} \omega_{3}\right)=0}}^{\prime} \\
& \times e^{-2 s \phi}\left[\left|\bar{\omega}_{1}+\bar{\omega}_{2} \bar{\lambda}+\bar{\omega}_{3} \bar{\gamma}\right|^{2}+e^{-2 \phi}\left|\bar{\omega}_{2}-i \bar{\omega}_{3} \lambda\right|^{2}+e^{-4 \phi}\left|\omega_{3}\right|^{2}\right]^{-s},
\end{aligned}
$$

which we recognize as the Eisenstein series $\mathcal{P}_{s}(\phi, \lambda, \gamma)$ constructed in Section 3.2.

\footnotetext{
${ }^{27}$ We note that the greatest common divisor in $\mathbb{Z}[i]$ is defined up to Gaussian units which are a subgroup of order 4 in the Gaussian integers $\mathbb{Z}[i]$. See Appendix A for more details on the Gaussian integers.
} 


\section{Appendix C. More on the Abelian measure}

This appendix contains a detailed analysis of the norm constraint (4.25) entering the Fourier expansion at various places and the derivation of the Abelian measure (4.68) as a sum over Gaussian divisors.

\section{C.1. Analysis of the norm constraint}

The norm constraint (4.25) requires to find, for a fixed integer $d$, all Gaussian integers with norm squared divisible by $2 d$. In this appendix we write this constraint as

$$
|\alpha|^{2}:=0 \bmod 2 d .
$$

C.1.1. Multiplicative structure. The solutions to the norm constraint possess a multiplicative structure. Let $d_{1}$ and $d_{2}$ be coprime integers and let $\alpha_{1}$ and $\alpha_{2}$ be Gaussian integers such that $2 d_{i}$ divides $\left|\alpha_{i}\right|^{2}$. Then clearly $\alpha_{1} \alpha_{2}$ satisfies the norm constraint for $d_{1} d_{2}$. Due to prime factorization of Gaussian integers we know that this describes all solutions and it is therefore sufficient to study the solutions to the norm constraint for powers of primes $d=p^{k}$. There are three qualitatively different cases.

(i) $p=2$, whence $d=2^{k}$. The structure of the set of solutions looks different for $k$ even and odd. For $k$ even one has that

$$
\alpha=2^{k / 2}\left(n_{1}+i n_{2}\right) \quad \text { for } n_{1}+n_{2} \in 2 \mathbb{Z}
$$

solves the constraint, whereas for $k$ odd

$$
\alpha=2^{(k+1) / 2}\left(n_{1}+i n_{2}\right)
$$

solves the constraint without restriction on the integers $n_{1}$ and $n_{2}$.

(ii) $p=4 n+3$. Again one has to distinguish $k$ even and $k$ odd in solving (C.1) for $d=p^{k}$. For $k$ even one has

$$
\alpha=p^{k / 2}\left(n_{1}+i n_{2}\right) \quad \text { for } n_{1}+n_{2} \in 2 \mathbb{Z}
$$

solves the constraint, whereas for $k$ odd

$$
\alpha=p^{(k+1) / 2}\left(n_{1}+i n_{2}\right) \quad \text { for } n_{1}+n_{2} \in 2 \mathbb{Z}
$$

solves the constraint. Note that there are restrictions on the integers $n_{1}$ and $n_{2}$ in both cases. 
(iii) $p=4 n+1$. This case is the most complicated one. Any such prime can be written as $p=a^{2}+b^{2}$ for some integers $a$ and $b$ and we assume $a>b$ without loss of generality. To describe the set of solutions to (C.1) for $d=p^{k}$ we again distinguish even and odd $k$. An important auxiliary definition is furnished by

$$
e_{k}=(a-i b)^{k}(1+i) \quad \Rightarrow \quad\left|e_{k}\right|^{2}=2 p^{k}
$$

providing an elementary solution of the constraint. With the help of the Gaussian integer $e_{k}$ one can define the following pairs of lattices for $k$ odd and $j=0, \ldots, \frac{k-1}{2}$ :

$$
\begin{aligned}
& \Lambda_{j+1}=\left\{p^{j}\left(k_{1} e_{k-2 j}+k_{2} i e_{k-2 j}\right): k_{1}, k_{2} \in \mathbb{Z}\right\}, \\
& \bar{\Lambda}_{j+1}=\left\{p^{j}\left(k_{1} \bar{e}_{k-2 j}+k_{2} i \bar{e}_{k-2 j}\right): k_{1}, k_{2} \in \mathbb{Z}\right\} .
\end{aligned}
$$

The set of all solutions for $k$ odd is then given by

$$
\bigcup_{j=0}^{(k-1) / 2}\left(\Lambda_{j+1} \cup \bar{\Lambda}_{j+1}\right) .
$$

For $k$ even one also requires the lattice

$$
\Lambda_{k+1}=\left\{p^{k / 2}\left(k_{1}+i k_{2}\right): k_{1}, k_{2} \in \mathbb{Z} \text { and } k_{1}+k_{2} \in 2 \mathbb{Z}\right\}
$$

and then all solutions are given by

$$
\bigcup_{j=0}^{k / 2-1}\left(\Lambda_{j+1} \cup \bar{\Lambda}_{j+1}\right) \cup \Lambda_{k+1} .
$$

Pictures of the three kinds of solution sets will be given momentarily when discussing the restriction to a fundamental domain under the action of a translation group.

C.1.2. Restriction to a fundamental domain. In the Abelian measure we made use of writing the solution to the constraint in terms of solutions in a fundamental domain in (4.40). We denote by

$$
\mathcal{F}(d)=\left\{\alpha \in \mathbb{Z}[i]:|\alpha|^{2}:=0 \bmod 2 d \text { and } 0 \leq \Re(\alpha)<d, 0 \leq \Im(\alpha)<2 d\right\}
$$


the set of solutions to (C.1) in the fundamental domain. From the analysis above we know that for $d_{1}$ and $d_{2}$ coprime, the following holds:

$$
\mathcal{F}\left(d_{1} d_{2}\right) \cong \mathcal{F}\left(d_{1}\right) \times \mathcal{F}\left(d_{2}\right)
$$

where the solutions are of the form $d_{2} f_{1}+d_{1} f_{2}$ for $f_{i} \in \mathcal{F}\left(d_{i}\right)$ up to translation by the lattice $L$ of (4.41) defining the fundamental domain. Therefore it is sufficient to restrict to $d=p^{k}$ being a power of a prime. For describing (C.11) more explicitly we have to make recourse to the results of the preceding section and distinguish three cases.

(i) $d=2^{k}$. Here one simply restricts the integers $n_{1}$ and $n_{2}$ in (C.2) and (C.3). The number of points in the fundamental domain is

$$
\sharp \mathcal{F}\left(2^{k}\right)=2^{k}=N\left(2^{k}\right)
$$

in agreement with (4.53). The easiest way of doing the counting is by computing the sizes of the fundamental cell of the lattices and comparing to the total area of the fundamental domain $2 d^{2}$. A typical lattice is depicted on the left of figure 1. For $k=1$ there are two points in the fundamental domain.

(ii) $d=p^{k}$ for $p=4 n+3$. The counting works similar, but one has to take into account the additional constraint in (C.5) leading to

$$
\sharp \mathcal{F}\left(p^{k}\right)=p^{2\lfloor k / 2\rfloor}=N\left(p^{k}\right) .
$$

The form of the lattice is identical to that of the left part of figure 1 . For $k=1$ there is only a single point $\alpha=0$ in the fundamental domain.

(iii) $d=p^{k}$ for $p=4 n+1$. The counting of points in the fundamental domain is now more involved since the individual lattices in (C.8), or (C.10), have common points that should not be overcounted. Each lattice has $p^{k}$ points but, for example, there are $p^{2 j}$ common points for the lattices $\Lambda_{j+1}$ and $\bar{\Lambda}_{j+1}$ forming the square lattice

$$
\Lambda_{j+1} \cap \bar{\Lambda}_{j+1}=\left\{p^{k-j}\left(k_{1}+i k_{2}\right): k_{1}+k_{2} \in 2 \mathbb{Z}\right\}
$$

implying

$$
\sharp\left(\left(\Lambda_{j+1} \cup \bar{\Lambda}_{j+1}\right) \cap \mathcal{F}(d)\right)=2 p^{k}-p^{2 j} .
$$



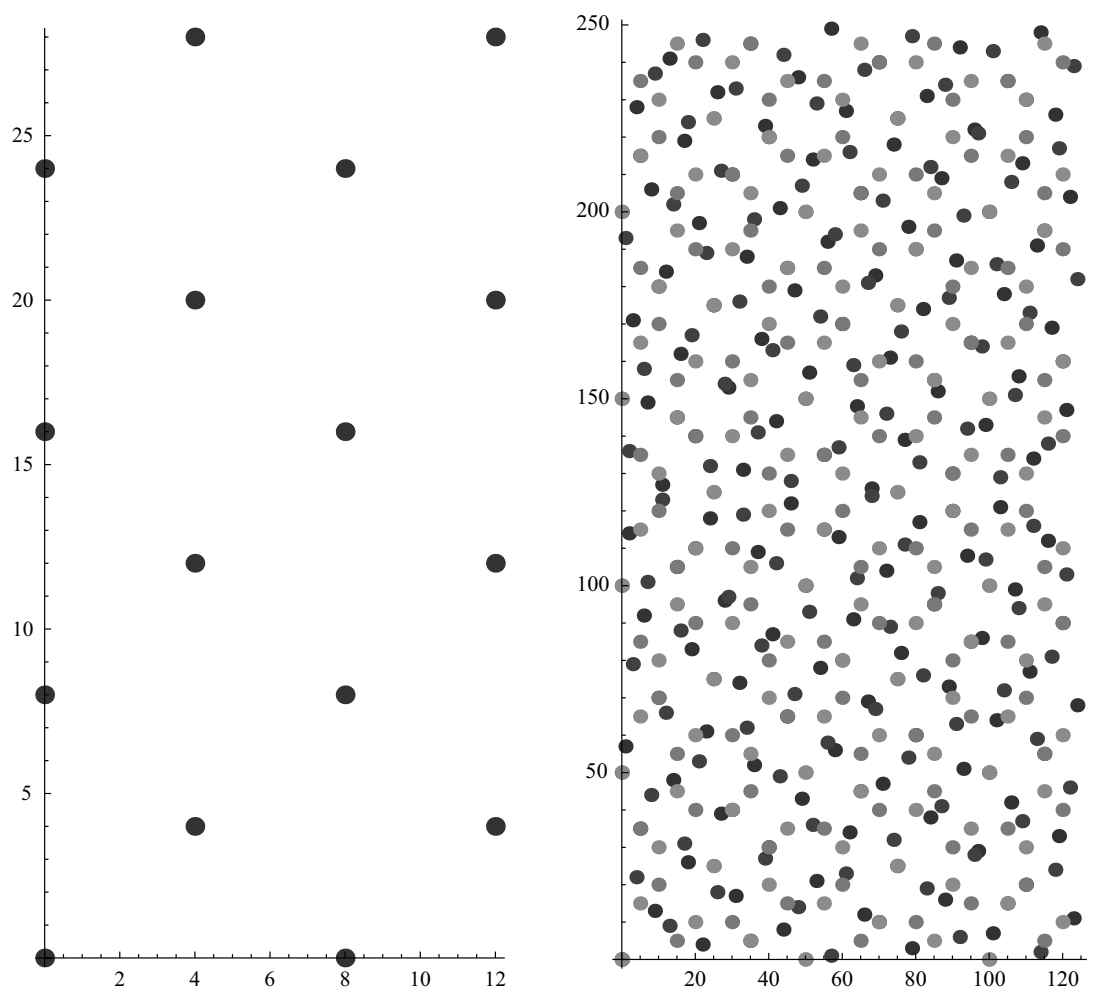

Figure 1: Left: The set $\mathcal{F}\left(2^{4}\right)$ is a $\pi / 4$ rotated and rescaled square lattice. Right: The set $\mathcal{F}\left(5^{3}\right)$ as the union of four lattices with common points.

One can also show that

$$
\sharp\left(\left(\Lambda_{j+1} \cup \bar{\Lambda}_{j+1}\right) \cap\left(\Lambda_{j+2} \cup \bar{\Lambda}_{j+2}\right) \cap \mathcal{F}(d)\right)=2 p^{k-1}-p^{2 j}
$$

and that all common points between pairs of lattices whose indices are farther apart are already contained in the intersection above. Putting everything together one arrives at the following count of points in the fundamental domain for $k$ odd:

$$
\begin{aligned}
\sharp \mathcal{F}\left(p^{k}\right) & =2 p^{k}-p^{0}+\sum_{j=1}^{(k-1) / 2}\left(2 p^{k}-p^{2 j}-\left(2 p^{k-1}-p^{2 j-2}\right)\right) \\
& =(k+1) p^{k}-k p^{k-1}=N\left(p^{k}\right) .
\end{aligned}
$$


For $k$ even the analysis is similar. An example of lattices with intersection points can be found on the right of figure 1.

\section{C.2. Rewriting the Abelian measure}

We now turn to deriving (4.68) from the Abelian measure (4.66). This involves mainly demonstrating the equality (4.67). To this end we introduce an additional function on the Gaussian integers

$$
\begin{aligned}
\nu_{s}(q) & =|q|^{2 s-2} \beta(2 s-1)\left(\sum_{d>0} d^{1-2 s} \sum_{f \in \mathcal{F}(d)} e^{\frac{\pi i}{d} \Re[q f(1-i)]}\right) \\
& =|q|^{2 s-2} \beta(2 s-1) \sum_{d>0} d^{1-2 s} a_{q}(d) .
\end{aligned}
$$

The function $\nu_{s}(q)$ is related to the 1.h.s. of (4.67) in an obvious way.

C.2.1. Evaluation of $\boldsymbol{a}_{\boldsymbol{q}}(\boldsymbol{d})$ and $\boldsymbol{\nu}_{\boldsymbol{s}}(\boldsymbol{q})$. The first observation is that the series $a_{q}(d)$ is multiplicative in $d$ for fixed $q$ (but not in $q$ ). This follows from (C.12) and a simple rewriting of the exponent. Therefore it is sufficient to determine $a_{q}(d)$ for $d=p^{k}$. This is where the description of the sets $\mathcal{F}\left(p^{k}\right)$ enters. The series $\nu_{s}(q)$ can be shown to be multiplicative so we only require $a_{q}(d)$ for $q=g^{m}$ as a power of a Gaussian prime and $d=p^{k}$ the power of standard prime.

The next observation is that

$$
a_{g^{m}}\left(p^{k}\right)=\sum_{f \in \mathcal{F}\left(p^{k}\right)} e^{\frac{\pi i}{p^{k}} \Re\left[g^{m} f(1-i)\right]}=\sum_{f \in g^{m} \mathcal{F}\left(p^{k}\right)} e^{\frac{\pi i}{p^{k}} \Re[f(1-i)]}
$$

by rotating (and rescaling) the set of fundamental solutions. If $g$ does not divide $p$ then the rotated set is an equivalently good fundamental set of solutions. Hence

$$
a_{g^{m}}\left(p^{k}\right)=a_{1}\left(p^{k}\right) \text { if } g \text { does not divide } p \text {. }
$$

For this reason we will first evaluate $a_{1}\left(p^{k}\right)$ and treat the case when $g$ divides $p$ afterwards.

It turns out that it suffices to count the number of times the lattice containing only the point $\alpha=0$ in $\mathcal{F}\left(p^{k}\right)$ appears in the sum over $\mathcal{F}\left(p^{k}\right)$. For 
all other lattices the sum over phases is zero. Hence one finds immediately

$$
a_{1}\left(2^{k}\right)=0 \quad \text { for } k>0
$$

and for $p=4 n+3$ that

$$
a_{1}\left(p^{k}\right)= \begin{cases}1, & k=1 \\ 0, & k>1\end{cases}
$$

For $p=4 n+1$ one has to count more carefully due to the intersection points. For $k=1$ the origin is the only common point in $\Lambda_{1}$ and $\bar{\Lambda}_{1}$ and hence is overcounted once leading to $a_{1}(p)=-1$. For $k>1$ this is offset by the intersection with $\Lambda_{2} \cup \bar{\Lambda}_{2}$, making $a_{1}\left(p^{k}\right)$ vanish. In total one has therefore for the Pythagorean primes

$$
a_{1}\left(p^{k}\right)= \begin{cases}-1, & k=1 \\ 0, & k>1\end{cases}
$$

Constructing the Dirichlet series in (C.19) via its Euler product therefore leads to, after referring back to (4.21),

$$
\sum_{d>0} d^{1-2 s} a_{1}(d)=\frac{1}{\beta(2 s-1)} \quad \Rightarrow \quad \nu_{s}(1)=1 .
$$

For $q=g^{m}$ one can perform a scaling of the lattices involved. Starting with the case of $g=1+i$ the value $p=2$ is important and one has

$$
(1+i)^{m} \mathcal{F}\left(2^{k}\right) \cong\left[\mathcal{F}\left(2^{k-m}\right)\right]^{2^{m}}
$$

defining the right-hand side to consist only of the origin for $k \leq m$. Counting now the number of times the origin appears leads to

$$
a_{(1+i)^{m}}\left(2^{k}\right)= \begin{cases}0, & \text { for } k>m, \\ 2^{k}=N\left(2^{k}\right), & \text { for } k \leq m .\end{cases}
$$

The corresponding auxiliary series (C.19) is then

$$
\begin{aligned}
\nu_{s}\left((1+i)^{m}\right) & =2^{m(s-1)} \beta(2 s-1)\left(1+\sum_{k=1}^{m} 2^{k(2-2 s)}\right) \sum_{d>0} d^{1-2 s} a_{1}(d), \\
& =\frac{1}{4} 2^{m(1-s)} \sum_{z \mid(1+i)^{m}}|z|^{4 s-4}
\end{aligned}
$$


and so is the usual divisor function multiplied by the proper overall factor to make it symmetric under $s \leftrightarrow 2-s$.

A similar analysis can be carried out for inert primes leading to

$$
a_{p^{m}}\left(p^{k}\right)= \begin{cases}0 & \text { for } k \geq 2(m+1) \\ p^{2\lfloor k / 2\rfloor}=N\left(p^{k}\right) & \text { for } k<2(m+1)\end{cases}
$$

Therefore the full result for (C.19) for inert primes is

$$
\begin{aligned}
\nu_{s}\left(p^{m}\right) & =p^{2 m(s-1)} \beta(2 s-1)\left(1+\sum_{k=1}^{2 m+1} p^{2\lfloor k / 2\rfloor+k(1-2 s)}\right) \frac{\sum_{d>0} d^{1-2 s} a_{1}(d)}{1+p^{1-2 s}} \\
(\mathrm{C} .30) & =\frac{1}{4} p^{2 m(1-s)} \sum_{z \mid p^{m}}|z|^{4 s-4} .
\end{aligned}
$$

For split primes one finds

$$
a_{g^{m}}\left(p^{k}\right)= \begin{cases}0 & \text { for } k>m+1 \\ -p^{m} & \text { for } k=m+1 \\ p^{k-1}(p-1) & \text { for } k<m+1\end{cases}
$$

Therefore the full result for split primes is

$$
\begin{aligned}
\nu_{s}\left(g^{m}\right) & =\frac{p^{m(s-1)}}{1-p^{1-2 s}}\left(1+\sum_{k=1}^{m}(p-1) p^{k-1+k(1-2 s)}-p^{m+(m+1)(1-2 s)}\right) \\
& =\frac{1}{4}|g|^{2 m(1-s)} \sum_{z \mid g^{m}}|z|^{4 s-4} .
\end{aligned}
$$

In summary the function $\nu_{s}(q)$ defined in (C.19) takes the value

$$
\nu_{s}(q)=\frac{1}{4}|q|^{2-2 s} \sum_{z \mid q}|z|^{4 s-4}
$$

for any Gaussian integer $q \neq 0$ and therefore is a Gaussian divisor function in disguise.

C.2.2. The Abelian instanton measure. The Abelian instanton measure of (4.66) is thus given by a sum over primitive divisors of $\Lambda=\ell_{2}-i \ell_{1}$

$$
\mu_{s}\left(\ell_{1}, \ell_{2}\right)=4 \sum_{\omega_{3}^{\prime} \mid \Lambda}|\Lambda|^{2-2 s} \nu_{s}\left(\frac{\Lambda}{\omega_{3}^{\prime}}\right)
$$

which, together with (C.33), leads to (4.68). 


\section{References}

[1] P. S. Aspinwall, Compactification, geometry and duality: $\mathcal{N}=2$ [arXiv:hep-th/0001001].

[2] C. M. Hull and P. K. Townsend, Unity of superstring dualities,' Nucl. Phys. B 438 (1995), 109 [arXiv:hep-th/9410167].

[3] E. Witten, String theory dynamics in various dimensions, Nucl. Phys. B 443 (1995), 85 [arXiv:hep-th/9503124].

[4] N. A. Obers and B. Pioline, U-duality and M-theory, Phys. Rept. 318 (1999), 113 [arXiv:hep-th/9809039].

[5] M. B. Green and M. Gutperle, Effects of D-instantons, Nucl. Phys. B 498 (1997), 195 [arXiv:hep-th/9701093].

[6] M. B. Green and J. H. Schwarz, Supersymmetrical String Theories, Phys. Lett. B 109 (1982), 444.

[7] D. J. Gross and E. Witten, Superstring modifications of Einstein's equations, Nucl. Phys. B 277 (1986), 1.

[8] E. D'Hoker, M. Gutperle and D. H. Phong, Two-loop superstrings and S-duality, Nucl. Phys. B 722 (2005), 81 [arXiv:hep-th/0503180].

[9] I. K. Kostov and P. Vanhove, Matrix string partition functions, Phys. Lett. B 444 (1998), 196 [arXiv:hep-th/9809130].

[10] G. W. Moore, N. Nekrasov and S. Shatashvili, D-particle bound states and generalized instantons, Commun. Math. Phys. 209 (2000), 77 [arXiv:hep-th/9803265].

[11] E. Kiritsis and B. Pioline, On $R^{4}$ threshold corrections in type IIB string theory and $(p, q)$ string instantons, Nucl. phys. B508, 509-534 (1997) [arXiv:hep-th/9707018].

[12] M. B. Green and P. Vanhove, D-instantons, strings and M-theory, Phys. Lett. B 408 (1997), 122 [arXiv:hep-th/9704145].

[13] N. Obers and B. Pioline, Eisenstein series and string thresholds, Commun. Math. Phys. 209 (2000), 275-324 [arXiv:hep-th/ 9903113].

[14] C. Vafa and E. Zaslow (eds.), Mirror Symmetry, Clay Math. Monogr., 1 (2003), 929pp. 
[15] S. Cecotti, S. Ferrara and L. Girardello, Geometry of Type II Superstrings and the Moduli of Superconformal Field Theories, Int. J. Mod. Phys. A 4 (1989), 2475.

[16] K. Becker, M. Becker and A. Strominger, Five-branes, membranes and nonperturbative string theory, Nucl. Phys. B456 (1995), 130-152 [arXiv:hep-th/9507158].

[17] I. Antoniadis, S. Ferrara, R. Minasian and K. S. Narain, $R^{4}$ couplings in $M$ - and type II theories on Calabi-Yau spaces, Nucl. Phys. B507 (1997), 571-588 [arXiv:hep-th/9707013].

[18] A. Strominger, Loop corrections to the universal hypermultiplet, Phys. Lett. B421 (1998), 139-148 [arXiv:hep-th/9706195].

[19] H. Gunther, C. Herrmann and J. Louis, Quantum corrections in the hypermultiplet moduli space, Fortsch. Phys. 48 (2000), 119 [arXiv:hepth/9901137].

[20] I. Antoniadis, R. Minasian, S. Theisen and P. Vanhove, String loop corrections to the universal hypermultiplet, Class. Quant. Grav. 20 (2003), 5079-5102 [arXiv:hep-th/0307268].

[21] N. Halmagyi, I. V. Melnikov and S. Sethi, Instantons, hypermultiplets and the heterotic string, JHEP 0707 (2007) 086 [arXiv:0704.3308 [hep-th]].

[22] S. M. Salamon, Quaternionic Kähler manifolds, Invent. Math. 67 (1) (1982), 143-171.

[23] A. Swann, Hyper-Kähler and quaternionic Kähler geometry, Math. Ann. 289 (3) (1991), 421-450.

[24] C. LeBrun, Fano manifolds, contact structures, and quaternionic geometry, Int. J. Math. 6 (3) (1995), 419-437 [arXiv:dg-ga/9409001].

[25] B. de Wit, B. Kleijn and S. Vandoren, Superconformal hypermultiplets, Nucl. Phys. B568 (2000), 475-502 [arXiv:hep-th/9909228].

[26] B. de Wit, M. Rocek and S. Vandoren, Hypermultiplets, hyperkähler cones and quaternion-Kähler geometry, JHEP 02, (2001), 039 [arXiv:hep-th/0101161].

[27] S. Alexandrov, B. Pioline, F. Saueressig and S. Vandoren, Linear perturbations of hyperkähler metrics, Lett. Math. Phys. 87 (2009), 225 [arXiv:0806.4620 [hep-th]]. 
[28] S. Alexandrov, B. Pioline, F. Saueressig and S. Vandoren, Linear perturbations of quaternionic metrics, Commun. Math. Phys. 296 (2010), 353 [arXiv:0810.1675 [hep-th]].

[29] S. Alexandrov, B. Pioline, F. Saueressig and S. Vandoren, D-instantons and twistors, JHEP 0903 (2009), 044 [arXiv:0812.4219 [hep-th]].

[30] D. Robles-Llana, M. Rocek, F. Saueressig, U. Theis and S. Vandoren, Nonperturbative corrections to $4 D$ string theory effective actions from $S L(2, \mathbb{Z})$ duality and supersymmetry, Phys. Rev. Lett. 98 (2007), 211602 [arXiv:hep-th/0612027].

[31] D. Robles-Llana, F. Saueressig, U. Theis and S. Vandoren, Membrane instantons from mirror symmetry, Commun. Num. Theor. Phys. 1 (2007), 681 [arXiv:0707.0838 [hep-th]].

[32] S. Alexandrov, D-instantons and twistors: some exact results, J. Phys. A 42 (2009), 335402 [arXiv:0902.2761 [hep-th]].

[33] S. Alexandrov and F. Saueressig, Quantum mirror symmetry and twistors, JHEP 0909 (2009), 108 [arXiv:0906.3743 [hep-th]].

[34] B. Pioline and D. Persson, The automorphic NS5-brane, Commun. Num. Theor. Phys. 3 (4) (2009), 697-754 [arXiv:0902.3274 [hep-th]].

[35] N. Yui, Update on the modularity of Calabi-Yau varieties, Fields Inst. Commun., 38 (2003), 307-362.

[36] P. Candelas, G. T. Horowitz, A. Strominger and E. Witten, Vacuum configurations for superstrings, Nucl. Phys. B 258 (1985), 46.

[37] A. Strominger and E. Witten, New Manifolds for superstring compactification, Commun. Math. Phys. 101 (1985), 341.

[38] P. Candelas, E. Derrick and L. Parkes, Generalized Calabi-Yau manifolds and the mirror of a rigid manifold, Nucl. Phys. B 407 (1993), 115 [arXiv:hep-th/9304045].

[39] M. Bershadsky, S. Cecotti, H. Ooguri and C. Vafa, Kodaira-Spencer theory of gravity and exact results for quantum string amplitudes, Commun. Math. Phys. 165 (1994), 311 [arXiv:hep-th/9309140].

[40] P. S. Aspinwall and J. Louis, On the ubiquity of K3 fibrations in string duality, Phys. Lett. B 369 (1996), 233 [arXiv:hep-th/9510234].

[41] S. Ferrara and S. Sabharwal, Dimensional reduction of type II superstrings, Class. Quant. Grav. 6 (1989), L77. 
[42] D. R. Morrison, Mirror symmetry and the type II string, Nucl. Phys. Proc. Suppl. 46 (1996), 146 [arXiv:hep-th/9512016].

[43] C. Schoen, Varieties dominated by product varieties, Int. J. Math. 7 (4) (1996), 541-571.

[44] G. W. Moore, Arithmetic and attractors, arXiv:hep-th/9807087.

[45] S. Gukov and C. Vafa, Rational conformal field theories and complex multiplication, Commun. Math. Phys. 246 (2004), 181 [arXiv:hepth/0203213].

[46] L. Bao, C. Colonnello, A. Kleinschmidt, B. E. W. Nilsson and D. Persson, Work in progress.

[47] K. Becker and M. Becker, Instanton action for type II hypermultiplets, Nucl. Phys. B551 (1999), 102-116 [arXiv:hep-th/9901126].

[48] N. Lambert and P. West, Duality groups, automorphic forms and higher derivative corrections, Phys. Rev. D75 (2007), 066002 [arXiv:hepth/0611318].

[49] L. Bao, M. Cederwall and B. E. W. Nilsson, Aspects of higher curvature terms and U-duality, Class. Quant. Grav. 25 (2008), 095001 [arXiv:0706.1183 [hep-th]].

[50] Y. Michel and B. Pioline, Higher derivative corrections, dimensional reduction and Ehlers duality, JHEP 0709 (2007), 103 [arXiv:0706.1769 [hep-th]].

[51] C. Colonnello and A. Kleinschmidt, Ehlers symmetry at the next derivative order, JHEP 0708 (2007), 078 [arXiv:0706.2816 [hep-th]].

[52] L. Bao, J. Bielecki, M. Cederwall, B. E. W. Nilsson and D. Persson, U-duality and the compactified Gauss-Bonnet term, JHEP 0807 (2008), 048 [arXiv:0710.4907 [hep-th]].

[53] B. de Wit, $N=2$ electric-magnetic duality in a chiral background, Nucl. Phys. Proc. Suppl. 49 (1996), 191 [arXiv:hep-th/9602060].

[54] R. Bohm, H. Gunther, C. Herrmann and J. Louis, Compactification of type IIB string theory on Calabi-Yau threefolds, Nucl. Phys. B 569 (2000), 229 [arXiv:hep-th/9908007].

[55] J. Manschot, Stability and duality in N=2 supergravity [arXiv:0906.1767 [hep-th]]. 
[56] E. Falbel, G. Francsics, P. D. Lax and J. R. Parker, Generators of a Picard modular group in two complex dimensions, arXiv:0911.1104 [math.CV].

[57] G. Francsics and P. D. Lax, A fundamental domain for the Picard modular group in $\mathbb{C}^{2}$, Vienna, Preprint ESI 1273 (2003).

[58] M. Gunaydin, K. Koepsell and H. Nicolai, The minimal unitary representation of $E_{8(8)}$, Adv. Theor. Math. Phys. 5 (2002), 923 [arXiv:hepth/0109005].

[59] M. Gunaydin and O. Pavlyk, Minimal unitary realizations of exceptional U-duality groups and their subgroups as quasiconformal groups, JHEP 0501 (2005), 019 [arXiv:hep-th/0409272].

[60] D. Kazhdan, B. Pioline and A. Waldron, Minimal representations, spherical vectors, and exceptional theta series. I, Commun. Math, Phys. 226 (2002), 1-40 [arXiv:hep-th/0107222].

[61] D. Kazhdan and A. Polishchuk, Minimal representations: spherical vectors and automorphic functionals [arXiv:math/0209315 [math.RT]].

[62] B. Pioline and A. Waldron, Automorphic forms: a physicist's survey, [arXiv:hep-th/0312068].

[63] M. Gunaydin, A. Neitzke, O. Pavlyk and B. Pioline, Quasiconformal actions, quaternionic discrete series and twistors: $S U(2,1)$ and $G_{2(2)}$, Commun. Math. Phys. 283 (2008), 169 [arXiv:0707.1669 [hep-th]].

[64] Y. Ishikawa, The generalized Whittaker functions for $S U(2,1)$ and the Fourier expansion of automorphic forms, J. Math. Sci. Univ. Tokyo 6 (1999), 477-526.

[65] R. P. Langlands, On the functional relations satisfied by Eisenstein series, Springer Lecture Notes in Mathematics, Vol. 544, 1-337, Springer, Berlin, 1976.

[66] T. Orloff, Dirichlet series and automorphic forms on unitary groups, Trans. Am. Math. Soc. 290 (2) (1985), 431-456.

[67] M. Henneaux, D. Persson and P. Spindel, Spacelike singularities and hidden symmetries of gravity, Living Rev. Rel. 11 (2008), 1 [arXiv:0710.1818 [hep-th]].

[68] D. Yasaki, Explicit reduction theory for $S U(2,1 ; \mathbb{Z}[i])$, [arXiv:math/ 0601071]. 
[69] I. Bars and Z. J. Teng, The unitary irreducible representations of SU(2,1), J. Math. Phys. 31 (1990), 1576.

[70] A. Borel, Automorphic form on $S L(2, \mathbb{R})$, 1st edn., Cambridge University Press, Cambridge, 1997.

[71] G. Zhang, Shimura invariant differential operators and their eigenvalues, Math. Ann. 319 (2) (2001), 235-265.

[72] A. I. Vinogradov and L. A. Takhtadzhyan, Theory of Eisenstein series for the group $S L(3, \mathbb{R})$ and its application to a binary problem, J. Sov. Math., 18 (3) (1983), 293.

[73] N. V. Proskurin, Expansions of automorphic functions, Translated from Zapiski Nauchnykh Seminarov Leningradskogo Otdeleniya Matematicheskogo Instituta im. V. A. Steklova AN USSR, 116 (1982), 119-141.

[74] P. Cartier, An introduction to zeta functions, in 'From Number Theory to Physics', eds. M. Waldschmidt et al., Springer, Berlin, 1995.

[75] Series A086933 in the Online encyclopedia of integer sequences, http://www.research.att.com/ njas/sequences/

[76] J. A. Wolf, Complex homogeneous contact manifolds and quaternionic symmetric spaces, J. Math. Mech. 14 (6) (1965), 1033-1047.

[77] L. Anguelova, M. Rocek and S. Vandoren, Quantum corrections to the universal hypermultiplet and superspace, Phys. Rev. D 70 (2004), 066001 [arXiv:hep-th/0402132].

[78] D. Robles-Llana, F. Saueressig and S. Vandoren, String loop corrected hypermultiplet moduli spaces, JHEP 0603 (2006), 081 [arXiv:hepth/0602164].

[79] S. Alexandrov, F. Saueressig and S. Vandoren, Membrane and fivebrane instantons from quaternionic geometry, JHEP, 09 (2006), 040 [arXiv:hep-th/0606259].

[80] M. Przanowski, Locally hermite-Einstein, self-dual gravitational instantons, Acta Phys. Polon. B 14 (8) (1983), 625-627.

[81] S. Alexandrov, B. Pioline and S. Vandoren, Self-dual Einstein Spaces, heavenly metrics and twistors, [arXiv:0912.3406 [hep-th]].

[82] B. Pioline and S. Vandoren, Large D-instanton effects in string theory, JHEP 0907 (2009), 008 [arXiv:0904.2303 [hep-th]]. 
[83] B. Pioline and A. Waldron, The automorphic membrane, JHEP 0406 (2004), 009 [arXiv:hep-th/0404018].

[84] N. A. Obers and B. Pioline, Exact thresholds and instanton effects in string theory, Fortsch. Phys. 49 (2001), 359 [arXiv:hep-th/0101122].

[85] B. H. Gross and N. R. Wallach, On quaternionic discrete series representations, and their continuations, J. Reine. Angew. Math. 481 (1996), $73-123$.

[86] R.-P. Holzapfel, Geometry and Arithmetic around Euler partial differential equations, D.Reidel Publishing Company, Springer, 1st edition, 1986 .

[87] E. Falbel and J. R. Parker, The geometry of the Eisenstein-Picard modular group, Duke Math. J. 131 (2) (2006), 249.

[88] T. .M. Apostol, Introduction to analytic number theory, Springer, Berlin, 1976.

[89] H. Neukirch, Algebraic number theory, Springer, Berlin, 2007.

[90] N. Koblitz, p-adic Numbers, p-adic analysis and zeta functions, Springer, Berlin, 1977.

[91] L. Brekke and P. G. O. Freund, p-adic numbers in physics, Phys. Rept. 233 (2003), 1-66.

[92] T. Shintani, On automorphic forms on unitary groups of order 3, (preprint, 1979).

Fundamental Physics, Chalmers University of Technology

SE-412 96, Göteborg, Sweden

E-mail address: ling.bao@chalmers.se, tfebn@chalmers.se,

daniel.persson@chalmers.se

Physique Théorique et Mathématique

Université Libre de Bruxelles \& International Solvay Institutes

UlB-Campus Plaine C.P. 231, B-1050 Bruxelles, Belgium

E-mail address: axel.kleinschmidt@ulb.ac.be, daniel.persson@chalmers.se

Laboratoire de Physique Théorique et Hautes Energies

CNRS UMR 7589 and Université Pierre et Marie Curie - Paris 6

4 Place Jussieu, 75252 Paris Cedex 05, France

E-mail address: pioline@lpthe.jussieu.fr

Received November 6, 2009 